\title{
Introduction to String Theory
}

\author{
Thomas Mohaupt \\ Friedrich-Schiller Universität Jena, Max-Wien-Platz 1, D-07743 Jena, Germany
}

\begin{abstract}
We give a pedagogical introduction to string theory, D-branes and p-brane solutions.
\end{abstract}

\section{Introductory remarks}

These notes are based on lectures given at the 271-th WE-Haereus-Seminar 'Aspects of Quantum Gravity'. Their aim is to give an introduction to string theory for students and interested researches. No previous knowledge of string theory is assumed. The focus is on gravitational aspects and we explain in some detail how gravity is described in string theory in terms of the graviton excitation of the string and through background gravitational fields. We include Dirichlet boundary conditions and D-branes from the beginning and devote one section to p-brane solutions and their relation to D-branes. In the final section we briefly indicate how string theory fits into the larger picture of M-theory and mention some of the more recent developments, like brane world scenarios.

The WE-Haereus-Seminar 'Aspects of Quantum Gravity' covered both main approaches to quantum gravity: string theory and canonical quantum gravity. Both are complementary in many respects. While the canonical approach stresses background independence and provides a non-perturbative framework, the cornerstone of string theory still is perturbation theory in a fixed background geometry. Another difference is that in the canonical approach gravity and other interactions are independent from each other, while string theory automatically is a unified theory of gravity, other interactions and matter. There is a single dimensionful constant and all couplings are functions of this constant and of vacuum expectation values of scalars. The matter content is uniquely fixed by the symmetries of the underlying string theory. Moreover, when formulating the theory in Minkowski space, the number of space-time dimensions is fixed. As we will see, there are only five distinct supersymmetric string theories in tendimensional Minkowski space.

The most important feature of string perturbation theory is the absence of UV divergencies. This allows one to compute quantum corrections to scattering amplitudes and to the effective action, including gravitational effects. More recently, significant progress has been made in understanding non-perturbative aspects of the theory, through the study of solitons and instantons, and through string dualities which map the strong coupling behaviour of one string theory to the weak coupling behaviour of a dual theory. Moreover, string dualities relate 
all five supersymmetric string theories to one another and lead to the picture of one single underlying theory, called M-theory. So far, only various limits of this theory are known, while the problem of finding an intrinsic, non-perturbative and background-independent definition is unsolved. One expects that M-theory has an underlying principle which unifies its various incarnations, presumably a symmetry principle. One of the obstacles on the way to the final theory is that it is not clear which degrees of freedom are fundamental. Besides strings, also higher-dimensional p-branes play an essential role. Moreover, there is an eleven-dimensional limit, which cannot be described in terms of strings.

Our presentation of string theory will be systematic rather than follow the path of historical development. Nevertheless we feel that a short historical note will be helpful, since many aspects which may seem somewhat ad hoc (such as the definition of interactions in section 3) become clearer in their historical context. The story started with the Veneziano amplitude, which was proposed as an amplitude for meson scattering in pre-QCD times. The amplitude fitted the known experimental data very well and had precisely the properties expected of a good scattering amplitude on the basis of S-matrix theory, the bootstrap program and Regge pole theory. In particular it had a very special soft UV behaviour. Later work by Y. Nambu, H.B. Nielsen and L. Susskind showed that the Veneziano amplitude, and various generalization thereof could be interpreted as describing the scattering of relativistic strings. But improved experimental data ruled out the Veneziano amplitude as a hadronic amplitude: it behaved just to softly in order to describe the hard, partonic substructures of hadrons seen in deep inelastic scattering. J. Scherk and J. Schwarz reinterpreted string theory as a unified theory of gravity and all other fundamental interactions, making use of the fact that the spectrum of a closed string always contains a massless symmetric tensor state which couples like a graviton. This lead to the development of perturbative string theory, as we will describe it in sections 2-4 of these lecture notes. More recently the perspective has changed again, after the role of D-branes, p-branes and string dualities was recognized. This will be discussed briefly in sections 5 and 6 .

From the historical perspective it appears that string theory is a theory which is 'discovered' rather than 'invented'. Though it was clear from the start that one was dealing with an interesting generalization of quantum field theory and general relativity, the subject has gone through several 'phase transitions', and its fundamental principles remain to be made explicit. This is again complementary to canonical quantum gravity, where the approach is more axiomatic, starting from a set of principles and proceeding to quantize Einstein gravity.

The numerous historical twists, our lack of final knowledge about the fundamental principles and the resulting diversity of methods and approaches make string theory a subject which is not easy to learn (or to teach). The 271-th WEHaereus-Seminar covered a broad variety of topics in quantum gravity, 'From Theory to Experimental Search'. The audience consisted of two groups: graduate students, mostly without prior knowledge of string theory, and researches, working on various theoretical and experimental topics in gravity. The two lec- 
tures on string theory were supposed to give a pedagogical introduction and to prepare for later lectures on branes worlds, large extra dimensions, the AdS-CFT correspondence and black holes. These lecture notes mostly follow the lectures, but aim to extend them in two ways. The first is to add more details to the topics I discussed in the lectures. In particular I want to expand on points which seemed to be either difficult or interesting to the audience. The second goal is to include more material, in order to bring the reader closer to the areas of current active research. Both goals are somewhat contradictory, given that the result is not meant to be a book, but lecture notes of digestable length. As a compromise I choose to explain those things in detail which seemed to be the most important ones for the participants of the seminar, hoping that they represent a reasonable sample of potential readers. On the other side several other topics are also covered, though in a more scetchy way. Besides summarizing advanced topics, which cannot be fully explained here, I try to give an overview of (almost) all the new developements of the last years and to indicate how they fit into the emerging overall picture of M-theory.

The outline of the lectures is as follows: sections $2-4$ are devoted to perturbative aspects of bosonic and supersymmetric string theories. They are the core of the lectures. References are given at the end of the sections. String theory has been a very active field over several decades, and the vast amount of existing literature is difficult to oversee even for people working in the field. I will not try to give a complete account of the literature, but only make suggestions for further reading. The basic references are the books [1,2, 3, 4, 5], which contain a huge number of references to reviews and original papers. The reader interested in the historical developement of the subject will find information in the annotated bibliography of [1]. Section 5 gives an introduction to non-perturbative aspects by discussing a particular class of solitons, the p-brane solutions of type II string theory. Section 6 gives an outlook on advanced topics: while sections 6.1-6.3 scetch how the five supersymmetric string theories fit into the larger picture of M-theory, section 6.4 gives an overview of current areas of research, together with references to lecture notes, reviews and some original papers.

\section{Free bosonic strings}

We start our study of string theories with the bosonic string. This theory is a toy-model rather than a realistic theory of gravity and matter. As indicated by its name it does not have fermionic states, and this disqualifies it as a theory of particle physics. Moreover, its groundstate in Minkowski space is a tachyon, i.e., a state of negative mass squared. This signals that the theory is unstable. Despite these shortcomings, the bosonic string has its virtues as a pedagogical toy-model: whereas we can postpone to deal with the additional techniques needed to describe fermions, many features of the bosonic string carry over to supersymmetric string theories, which have fermions but no tachyon. 


\subsection{Classical bosonic strings}

We start with a brief overview of classical aspects of bosonic strings.

Setting the stage. Let us first fix our notation. We consider a fixed background Pseudo-Riemannian space-time $\mathcal{M}$ of dimension $D$, with coordinates $X=\left(X^{\mu}\right)$, $\mu=0, \ldots, D-1$. The metric is $G_{\mu \nu}(X)$ and we take the signature to be 'mostly plus', $(-)(+)^{D-1}$.

The motion of a relativistic string in $\mathcal{M}$ is described by its generalized worldline, a two-dimensional surface $\Sigma$, which is called the world-sheet. For a single non-interacting string the world-sheet has the form of an infinite strip. We introduce coordinates $\sigma=\left(\sigma^{0}, \sigma^{1}\right)$ on the world-sheet. The embedding of the world-sheet into space-time is given by maps

$$
X: \Sigma \longrightarrow \mathcal{M}: \sigma \longrightarrow X(\sigma) \text {. }
$$

The background metric induces a metric on the world-sheet:

$$
G_{\alpha \beta}=\frac{\partial X^{\mu}}{\partial \sigma^{\alpha}} \frac{\partial X^{\nu}}{\partial \sigma^{\beta}} G_{\mu \nu},
$$

where $\alpha, \beta=0,1$ are world-sheet indices. The induced metric is to be distinguished from an intrinsic metric $h_{\alpha \beta}$ on $\Sigma$. As we will see below, an intrinsic metric is used as an auxiliary field in the Polyakov formulation of the bosonic string.

A useful, but sometimes confusing fact is that the above setting can be viewed from two perspectives. So far we have taken the space-time perspective, interpreting the system as a relativistic string moving in space-time $\mathcal{M}$. Alternatively we may view it as a two-dimensional field theory living on the world-sheet, with fields $X$ which take values in the target-space $\mathcal{M}$. This is the world-sheet perspective, which enables us to use intuitions and methods of two-dimensional field theory for the study of strings.

Actions. The natural action for a relativistic string is its area, measured with the induced metric:

$$
S_{\mathrm{NG}}=\frac{1}{2 \pi \alpha^{\prime}} \int_{\Sigma} d^{2} \sigma\left|\operatorname{det} G_{\alpha \beta}\right|^{1 / 2} .
$$

This is the Nambu-Goto action, which is the direct generalization of the action for a massive relativistic particle. The prefactor $\left(2 \pi \alpha^{\prime}\right)^{-1}$ is the energy per length or tension of the string, which is the fundamental dimensionful parameter of the theory. We have expressed the tension in terms of the so-called Regge slope $\alpha^{\prime}$, which has the dimension (length) ${ }^{2}$ in natural units, $c=1, \hbar=1$. Most of the time we will use string units, where in addition we set $\alpha^{\prime}=\frac{1}{2}$.

The Nambu-Goto action has a direct geometric meaning, but is technically inconvenient, due to the square root. Therefore one prefers to use the Polyakov 
action, which is equivalent to the Nambu-Goto action, but is a standard twodimensional field theory action. In this approach one introduces an intrinsic metric on the world-sheet, $h_{\alpha \beta}(\sigma)$, as additional datum. The action takes the form of a non-linear sigma-model on the world-sheet,

$$
S_{\mathrm{P}}=\frac{1}{4 \pi \alpha^{\prime}} \int_{\Sigma} d^{2} \sigma \sqrt{h} h^{\alpha \beta} \partial_{\alpha} X^{\mu} \partial_{\beta} X^{\nu} G_{\mu \nu}(X),
$$

where $h=\left|\operatorname{det} h_{\alpha \beta}\right|$.

The equation of motion for $h_{\alpha \beta}$ is algebraic. Thus the intrinsic metric is non-dynamical and can be eliminated, which brings us back to the Nambu-Goto action. Since

$$
T_{\alpha \beta}:=\left(2 \pi \alpha^{\prime} \sqrt{h}\right)^{-1} \frac{\delta S_{\mathrm{P}}}{\delta h^{\alpha \beta}}=\partial_{\alpha} X^{\mu} \partial_{\beta} X_{\mu}-\frac{1}{2} h_{\alpha \beta} \partial_{\gamma} X^{\mu} \partial^{\gamma} X_{\mu}
$$

is the energy momentum of the two-dimensional field theory defined by (㺼), we can interpret the equation of motion of $h_{\alpha \beta}$ as the two-dimensional Einstein equation. The two-dimensional metric is non-dynamical, because the two-dimensional Einstein-Hilbert action is a topological invariant, proportional to the Euler number of $\Sigma$. Thus its variation vanishes and the Einstein equation of (雨) coupled to two-dimensional gravity reduces to $T_{\alpha \beta}=0$. Note that the energy-momentum tensor (5) is traceless, $h^{\alpha \beta} T_{\alpha \beta}=0$. This holds before imposing the equations of motion ('off shell'). Therefore $T_{\alpha \beta}$ has only two independent components, which vanish for solutions to the equations of motion ('on shell'). Since the trace of the energy-momentum tensor is the Noether current of scale transformations, this shows that the two-dimensional field theory (何) is scale invariant. As we will see below, it is in fact a conformal field theory.

The Polyakov action has three local symmetries. Two are shared by the Nambu-Goto action, namely reparametrizations of the world-sheet:

$$
\sigma^{\alpha} \longrightarrow \tilde{\sigma}^{\alpha}\left(\sigma^{0}, \sigma^{1}\right) \text {. }
$$

The third local symmetry is the multiplication of the metric $h_{\alpha \beta}$ by a local, positive scale factor,

$$
h_{\alpha \beta}(\sigma) \longrightarrow e^{\Lambda(\sigma)} h_{\alpha \beta}(\sigma) \text {. }
$$

This transformation is called a Weyl transformation by physicists, while mathematicians usually use the term conformal transformation. The three local symmetries can be used to gauge-fix the metric $h_{\alpha \beta}$. The standard choice is the conformal gauge,

$$
h_{\alpha \beta}(\sigma) \stackrel{!}{=} \eta_{\alpha \beta}, \quad \text { where }\left(\eta_{\alpha \beta}\right)=\operatorname{Diag}(-1,1) .
$$

While this gauge can be imposed globally on the infinite strip describing the motion of a single non-interacting string, it can only be imposed locally on more general world-sheets, which describe string interactions. We will discuss global aspects of gauge fixing later. 
The conformal gauge does not provide a complete gauge fixing, because (8) is invariant under a residual symmetry. One can still perform reparametrizations under which the metric only changes by a local, positive scale factor, because this factor can be absorbed by a Weyl transformation. Such conformal reparametrizations are usually called conformal transformations by physicists. Note that the same term is used for Weyl transformations by mathematicians. A convenient way to characterize conformal reparametrizations in terms of coordinates is to introduce light cone coordinates,

$$
\sigma^{ \pm}=\sigma^{0} \pm \sigma^{1}
$$

Then conformal reparametrization are precisely those reparametrizations which do not mix the light cone coordinates:

$$
\sigma^{+} \longrightarrow \tilde{\sigma}^{+}\left(\sigma^{+}\right), \quad \sigma^{-} \longrightarrow \tilde{\sigma}^{-}\left(\sigma^{-}\right)
$$

Thus we are left with an infinite-dimensional group of symmetries, which in particular includes scale transformations.

Equations of motion, closed and open strings, and D-branes. In order to proceed we now spezialize to the case of a flat space-time, $G_{\mu \nu}=\eta_{\mu \nu}$, where $\eta_{\mu \nu}=\operatorname{Diag}(-1,+1, \ldots,+1)$. In the conformal gauge the equation of motion for $X$ reduces to a free two-dimensional wave equation,

$$
\partial^{2} X^{\mu}=\partial^{\alpha} \partial_{\alpha} X^{\mu}=0 .
$$

Note that when imposing the conformal gauge on the Polyakov action (4), the equation of motion for $h_{\alpha \beta}$, i.e., $T_{\alpha \beta}=0$, becomes a constraint, which has to be imposed on the solutions of (11).

The general solution of (11) is a superposition of left- and right-moving waves,

$$
X^{\mu}(\sigma)=X_{L}^{\mu}\left(\sigma^{+}\right)+X_{R}^{\mu}\left(\sigma^{-}\right) .
$$

However, we also have to specify boundary conditions at the ends of the string. One possible choice are periodic boundary conditions,

$$
X^{\mu}\left(\sigma^{0}, \sigma^{1}+\pi\right)=X^{\mu}\left(\sigma^{0}, \sigma^{1}\right) .
$$

They correspond to closed strings. A convient parametrization of the solution is:

$$
X^{\mu}(\sigma)=x^{\mu}+2 \alpha^{\prime} p^{\mu} \sigma^{0}+\mathrm{i} \sqrt{2 \alpha^{\prime}} \sum_{n \neq 0} \frac{\alpha_{n}^{\mu}}{n} e^{-2 \mathrm{i} n \sigma^{+}}+\mathrm{i} \sqrt{2 \alpha^{\prime}} \sum_{n \neq 0} \frac{\tilde{\alpha}_{n}^{\mu}}{n} e^{-2 \mathrm{i} n \sigma^{-}} .
$$

Reality of $X^{\mu}$ implies: $\left(x^{\mu}\right)^{\star}=x^{\mu}$ and $\left(p^{\mu}\right)^{\star}=p^{\mu}$ and $\left(\alpha_{m}^{\mu}\right)^{\star}=\alpha_{-m}^{\mu}$ and $\left(\tilde{\alpha}_{m}^{\mu}\right)^{\star}=\tilde{\alpha}_{-m}^{\mu}$. Here $\star$ denotes complex conjugation. While $x^{\mu}$ is the position of the center of mass of the string at time $\sigma^{0}, p^{\mu}$ is its total momentum. Thus, the center of mass moves on a straight line in Minkowski space, like a free relativistic 
particle. The additional degrees of freedom are decoupled left- and right-moving waves on the string, with Fourier components $\alpha_{m}^{\mu}$ and $\tilde{\alpha}_{m}^{\mu}$.

When not choosing periodic boundary conditions, the world-sheet has boundaries and we have open strings. The variation of the world-sheet action yields a boundary term, $\delta S \simeq \int_{\partial \Sigma} d \sigma^{0} \partial_{1} X^{\mu} \delta X_{\mu}$. The natural choice to make the boundary term vanish are Neumann boundary conditions,

$$
\left.\partial_{1} X^{\mu}\right|_{\sigma^{1}=0}=0,\left.\quad \partial_{1} X^{\mu}\right|_{\sigma^{1}=\pi}=0
$$

With these boundary conditions, momentum is conserved at the ends of the string. Left- and right-moving waves are reflected at the ends and combine into standing waves. The solution takes the form

$$
X^{\mu}(\sigma)=x^{\mu}+\left(2 \alpha^{\prime}\right) p^{\mu} \sigma^{0}+\mathrm{i} \sqrt{2 \alpha^{\prime}} \sum_{n \neq 0} \frac{\alpha_{n}^{\mu}}{n} e^{-\mathrm{i} n \sigma^{0}} \cos \left(n \sigma^{1}\right) .
$$

There is, however, a second possible choice of boundary conditions for open strings, namely Dirichlet boundary conditions. Here the ends of the string are kept fixed:

$$
\left.X^{\mu}\right|_{\sigma^{1}=0}=x_{(1)}^{\mu},\left.\quad X^{\mu}\right|_{\sigma^{1}=\pi}=x_{(2)}^{\mu} .
$$

With these boundary conditions the solution takes the form

$$
X^{\mu}(\sigma)=x_{(1)}^{\mu}+\left(x_{(2)}^{\mu}-x_{(1)}^{\mu}\right) \frac{\sigma^{1}}{\pi}+\mathrm{i} \sqrt{2 \alpha^{\prime}} \sum_{n \neq 0} \frac{\alpha_{n}^{\mu}}{n} e^{-\mathrm{i} n \sigma^{0}} \sin \left(n \sigma^{1}\right) .
$$

More generally we can impose Neumann boundary conditions in the time and in $\mathrm{p}$ space directions and Dirichlet boundary conditions in the other directions. Let us denote the Neumann directions by $\left(X^{m}\right)=\left(X^{0}, X^{1}, \ldots, X^{p}\right)$ and the Dirichlet directions by $\left(X^{a}\right)=\left(X^{p+1}, \ldots, X^{D-1}\right)$.

The most simple choice of Dirichlet boundary conditions is then to require that all open strings begin and end on a p-dimensional plane located at an arbitrary position $X^{a}=x_{(1)}^{a}$ along the Dirichlet directions. Such a plane is called a p-dimensional Dirichlet-membrane, or D-p-brane, or simply D-brane for short. While the ends of the strings are fixed in the Dirichlet directions, they still can move freely along the Neumann directions. The world-volume of a D-p-brane is $(p+1)$-dimensional. The Neumann directions are called the world-volume or the parallel directions, while the Dirichlet directions are called transverse directions.

An obvious generalization is to introduce $N>1$ such D-p-branes, located at positions $x_{(i)}^{a}$, where $i=1, \ldots, N$, and to allow strings to begin and end on any of these. In this setting the mode expansion for a string starting on the i-th D-brane and ending on the $\mathrm{j}$-th is:

$$
\begin{aligned}
& X^{m}(\sigma)=x^{m}+\left(2 \alpha^{\prime}\right) p^{m} \sigma^{0}+\mathrm{i} \sqrt{2 \alpha^{\prime}} \sum_{n \neq 0} \frac{\alpha_{n}^{m}}{n} e^{-\mathrm{i} n \sigma^{0}} \cos \left(n \sigma^{1}\right) \\
& X^{a}(\sigma)=x_{(i)}^{a}+\left(x_{(j)}^{a}-x_{(i)}^{a}\right) \frac{\sigma^{1}}{\pi}+\mathrm{i} \sqrt{2 \alpha^{\prime}} \sum_{n \neq 0} \frac{\alpha_{n}^{a}}{n} e^{-\mathrm{i} n \sigma^{0}} \sin \left(n \sigma^{1}\right) .
\end{aligned}
$$


(One might also wonder about Dirichlet boundary conditions in the time direction. This makes sense, at least for Euclidean space-time signature, and leads to instantons, called D-instantons, which we will not discuss in these lectures.)

Dirichlet boundary conditions have been neglected for several years. The reason is that momentum is not conserved at the ends of the strings, reflecting that translation invariance is broken along the Dirichlet directions. Therefore, in a complete fundamental theory the D-branes must be new dynamical objects, different from strings. The relevance of such objects was only appreciated when it became apparent that string theory already includes solitonic space-time backgrounds, so called ('RR-charged') p-Branes, which correspond to D-branes. We will return to this point later.

Promoting the D-branes to dynamical objects implies that they will interact through the exchange of strings. This means that in general they will repulse or attract, and therefore their positions become dynamical. But there exist many static configurations of D-branes (mainly in supersymmetric string theories), where the attractive and repulsive forces cancel for arbitrary distances of the branes.

\subsection{Quantized bosonic strings}

The definition of a quantum theory of bosonic strings proceeds by using standard recipies of quantization. The two most simple ways to proceed are called 'old covariant quantization' and 'light cone quantization'. As mentioned above imposing the conformal gauge leaves us with a residual gauge invariance. In light cone quantization one fixes this residual invariance by imposing the additional condition

$$
X^{+} \stackrel{!}{=} x^{+}+p^{+} \sigma^{+}, \text {i.e. }, \alpha_{m}^{+} \stackrel{!}{=} 0
$$

where $X^{ \pm}=\frac{1}{\sqrt{2}}\left(X^{0} \pm X^{D-1}\right)$ are light cone coordinates in space-time. Then the constraints $T_{\alpha \beta}=0$ are solved in the classical theory. This yields (nonlinear) expressions for the oscillators $\alpha_{n}^{-}$in terms of the transverse oscillators $\alpha_{n}^{i}, i=1, \ldots D-2$. In light cone coordinates the world-sheet is embedded into space-time along the $X^{0}, X^{D-1}$ directions. The independent degrees of freedom are the oscillations transverse to the world sheet, which are parametrized by the $\alpha_{n}^{i}$. One proceeds to quantize these degrees of freedom. In this approach unitarity of the theory is manifest, but Lorentz invariance is not.

In old covariant quantization one imposes the constraints at the quantum level. Lorentz covariance is manifest, but unitarity is not: one has to show that there is a positive definite space of states and an unitary S-matrix. This is the approach we will describe in more detail below.

One might also wonder about 'new covariant quantization', which is BRST quantization. This approach is more involved but also more powerful then old covariant quantization. When dealing with advanced technical problems, for example the construction of scattering amplitudes involving fermions in superstring theories, BRST techniques become mandatory. But this is beyond the scope of these lectures. 
The Fock space. The first step is to impose canonical commutation relations on $X^{\mu}(\sigma)$ and its canonical momentum $\Pi^{\mu}(\sigma)=\partial_{0} X^{\mu}(\sigma)$. In terms of modes one gets

$$
\left[x^{\mu}, p^{\nu}\right]=i \eta^{\mu \nu}, \quad\left[\alpha_{m}^{\mu}, \alpha_{n}^{\nu}\right]=m \eta^{\mu \nu} \delta_{m+n, 0} .
$$

For closed strings there are analogous relations for $\tilde{\alpha}_{m}^{\mu}$. The reality conditions of the classical theory translate into hermiticity relations:

$$
\left(x^{\mu}\right)^{+}=x^{\mu}, \quad\left(p^{\mu}\right)^{+}=p^{\mu}, \quad\left(\alpha_{m}^{\mu}\right)^{+}=\alpha_{-m}^{\mu} .
$$

While the commutation relations for $x^{\mu}, p^{\nu}$ are those of a relativistic particle, the $\alpha_{m}^{\mu}$ satisfy the relations of creation and annihilation operators of harmonic oscillators, though with an unconventional normalization.

To proceed, one constructs a Fock space $\mathcal{F}$ on which the commutation relations (21) are repesented. First one chooses momentum eigenstates $|k\rangle$, which are annihiliated by half of the oscillators:

$$
p^{\mu}|k\rangle=k^{\mu}|k\rangle, \quad \alpha_{m}^{\mu}|k\rangle=0=\tilde{\alpha}_{m}^{\mu}|k\rangle, \quad m>0 .
$$

Then a basis $\mathcal{B}$ of $\mathcal{F}$ is obtained by acting with creation operators:

$$
\mathcal{B}=\left\{\alpha_{-m_{1}}^{\mu_{1}} \cdots \tilde{\alpha}_{-n_{1}}^{\nu_{1}} \cdots|k\rangle \mid m_{l}, n_{l}>0\right\} .
$$

A bilinear form on $\mathcal{F}$, which is compatible with the hermiticity properties (22), cannot be positive definite. Consider for example the norm squared of the state $\alpha_{-m}^{\mu}|k\rangle$ :

$$
\left\langle k\left|\left(\alpha_{-m}^{\mu}\right)^{+} \alpha_{-m}^{\mu}\right| k\right\rangle \sim \eta^{\mu \mu}= \pm 1 .
$$

However, the Fock space is not the space of physical states, because we still have to impose the constraints. The real question is whether the subspace of physical states contains states of negative norm.

The Virasoro Algebra. Constraints arise when the canonical momenta of a system are not independent. This is quite generic for relativistic theories. The most simple example is the relativistic particle, where the constraint is the mass shell condition, $p^{2}+m^{2}=0$. When quantizing the relativistic particle, physical states are those annihilated by the constraint, i.e., states satisfying the mass shell condition:

$$
\left(p^{2}+m^{2}\right)|\Phi\rangle=0 .
$$

When evaluating this in a basis of formal eigenstates of the operator $x^{\mu}$, one obtains the Klein-Gordon equation, $\left(\partial^{2}+m^{2}\right) \Phi(x)=0$, where $\Phi(x)=\langle x \mid \Phi\rangle$ is interpreted as the state vector in the $x$-basis. This is a clumsy way to approach the quantum theory of relativistic particles, and one usually prefers to use quantum field theory ('second quantization') rather than quantum mechanics ('first quantization'). But in string theory it turns out that the first quantized formulation works nicely for studying the spectrum and computing amplitudes, whereas string field theory is very complicated. 
Proceeding parallel to the case of a relativistic particle one finds that the canonical momentum is $\Pi^{\mu}=\partial_{0} X^{\mu}$. The constraints are

$$
\Pi^{\mu} \partial_{1} X_{\mu}=0, \quad \Pi^{\mu} \Pi_{\mu}+\partial_{1} X^{\mu} \partial_{1} X_{\mu}=0 .
$$

In the Polyakov formulation they are equivalent to $T_{\alpha \beta}=0$. It is convenient to express the constraints through the Fourier components of $T_{\alpha \beta}$. Passing to light cone coordinates, the tracelessness of $T_{\alpha \beta}$, which holds without using the equation of motion or imposing the constraints, implies

$$
T_{+-}=0=T_{-+} .
$$

Thus we are left with two independent components, $T_{++}$and $T_{--}$, where $T_{ \pm \pm} \simeq$ $\partial_{ \pm} X^{\mu} \partial_{ \pm} X_{\mu}$. For closed strings, where $\partial_{ \pm} X^{\mu}$ are periodic in $\sigma^{1}$, we expand $T_{ \pm \pm}$ in a Fourier series and obtain Fourier coefficients $L_{m}, \tilde{L}_{m}, m \in \mathbf{Z}$. For open strings, observe that $\sigma^{1} \rightarrow-\sigma^{1}$ exchanges $\partial_{+} X^{\mu}$ and $\partial_{-} X^{\mu}$. Both fields can be combined into a single field, which is periodic on a formally doubled worldsheet with $-\pi \leq \sigma^{1} \leq \pi$. In the same way one can combine $T_{++}$with $T_{--}$. By Fourier expansion on the doubled world-sheet one then obtains one set of Fourier modes for the energy-momentum tensor, denoted $L_{m}$. This reflects that left- and right-moving waves couple through the boundaries.

The explicit form for the $L_{m}$ in terms of oscillators is

$$
L_{m}=\frac{1}{2} \sum_{n=-\infty}^{\infty} \alpha_{m-n} \cdot \alpha_{n},
$$

with an analogous formula for $\tilde{L}_{m}$ for closed strings. We have denoted the contraction of Lorentz indices by '.' and defined $\alpha_{0}^{\mu}=\frac{1}{2} p^{\mu}=\tilde{\alpha}_{0}^{\mu}$ for closed strings and $\alpha_{0}^{\mu}=p^{\mu}$ for open strings. In terms of the Fourier modes, the constraints are $L_{m}=0$, and, for closed string, $\tilde{L}_{m}=0$. Translations in $\sigma^{0}$ are generated by $L_{0}$ for open and by $L_{0}+\tilde{L}_{0}$ for closed strings. These functions are the world-sheet Hamiltonians. The $L_{m}$ satisfy the Witt algebra algebra,

$$
\left\{L_{m}, L_{n}\right\}_{\text {P.B. }}=i(m-n) L_{m+n},
$$

where $\{\cdot, \cdot\}_{\text {P.B. }}$. is the Poisson bracket. For closed strings we have two copies of this algebra. The Witt algebra is the Lie algebra of infinitesimal conformal transformations. Thus the constraints reflect that we have a residual gauge symmetry corresponding to conformal transformations. Since the constraints form a closed algebra with the Hamiltonian, they are preserved in time. Such constraints are called first class, and they can be imposed on the quantum theory without further modifications (such as Dirac brackets).

In the quantum theory the $L_{m}$ are taken to be normal ordered, i.e., annihilation operators are moved to the right. This is unambigous, except for $L_{0}$. We will deal with this ordering ambiguity below. The hermiticiy properties of the $L_{m}$ are:

$$
L_{m}^{+}=L_{-m}
$$


The operators $L_{m}$ satisfy the Virasora algebra:

$$
\left[L_{m}, L_{n}\right]=(m-n) L_{m+n}+\frac{c}{12}\left(m^{3}-m\right) \delta_{m+n, 0} .
$$

The Virasoro algebra is a central extension of the Witt algebra. On our Fock space $\mathcal{F}$ the central charge $c$ takes the value

$$
c=\eta^{\mu \nu} \eta_{\mu \nu}=D,
$$

i.e., each space-time dimension contributes one unit. Since the Poisson brackets of $L_{m}$ in the classical theory just give the Witt algebra, this dependence on the number of dimensions is a new property of the quantum theory. The extra central term occuring at the quantum level is related to a normal ordering ambiguity of commutators with $m+n=0$. This results in a new 'anomalous' term in the algebra. In the context of current algebras such terms are known as Schwinger terms.

Imposing the constraints, or, why $D=26$ ? In the classical theory the constraints amount to imposing $L_{m}=0$ on solutions. Imposing this as an operator equation on the quantum theory is too strong. In particular it is not compatible with the algebra (32). What can be imposed consistently is that matrix elements of the $L_{m}$ vanish between physical states, $\left\langle\Phi_{1}\left|L_{m}\right| \Phi_{2}\right\rangle=0$. Conversely this condition singles out the subspace of physical states, $\mathcal{F}_{\text {phys }} \subset \mathcal{F}$. Using the hermiticity properties of the $L_{m}$, this is equivalent to the statement that the positive Virasoro modes annihilate physical states,

$$
\begin{aligned}
& L_{m}|\Phi\rangle=0, \quad m>0, \\
& \left(L_{0}-a\right)|\Phi\rangle=0,
\end{aligned}
$$

for all $|\Phi\rangle \in \mathcal{F}_{\text {phys }}$. Note that we have introduced an undetermined constant $a$ into the $L_{0}$-constraint. As mentioned above this operator has an ordering ambiguity. We take $L_{0}$ to be normal ordered and parametrize possible finite ordering effects by the constant $a$. Since $L_{0}$ is the Hamiltonian, this might be considered as taking into account a non-trivial Casimir effect. In the case of closed strings there is a second set of constraints involving the $\tilde{L}_{m}$.

The Virasoro operators $L_{-m}, m>0$ still act non-trivially on physical states and create highest weight representations of the Virasoro algebra. This corresponds to the fact that we still have residual gauge symmetries. Therefore it is clear that $\mathcal{F}_{\text {phys }}$ is not the physical Hilbert space. $\mathcal{F}_{\text {phys }}$ is not positive definite, but contains null states (states of norm zero) and, depending on the number of space-time dimensions, also states of negative norm. A positive definite space of states can be constructed if negative norm states are absent, such that $\mathcal{F}_{\text {phys }}$ is positive semi-definite, and if null states are orthogonal to all physical states. Then one can consistently identify physical states $|\Phi\rangle$ that differ by null states $|\Psi\rangle$,

$$
|\Phi\rangle \simeq|\Phi\rangle+|\Psi\rangle
$$


and define the Hilbert space by

$$
\mathcal{H}=\mathcal{F}_{\text {phys }} /\{\text { Null states }\} .
$$

The working of this construction crucially depends on the values of $D$ and $a$. This is the contents of the so-called no-ghost theorem, which can be summarized as follows:

1. $D=26$ and $a=1$. The construction works as described above. The resulting theory is known as the critical (bosonic) string theory, $D=26$ is the critical dimension. Physical states differing by a null states differ by a residual gauge transformation and represent the same state in the Hilbert space. We will see explicit examples below.

2. $D>26$. The physical subspace $\mathcal{F}_{\text {phys }}$ always contains states of negative norm and no Hilbert space $\mathcal{H}$ can be constructed. There is no bosonic string theory for $D>26$.

3. $D \leq 25$. Naively one expects such theories to be unitary, because we can just truncate the unitary critical string theory and this cannot introduce states of negative norm. Nevertheless one does not obtain a consistent quantum theory by truncation. When studying scattering amplitudes at the loop level one finds poles corresponding to unphysical negative norm states and there is no unitary S-matrix. Thus truncations of the critical string do not yield unitary theories.

But there is an alternative to truncation, known as Liouville string theory or non-critical string theory. This theory exists in $D<26$, at the price that the quantum theory has a new degree of freedom, the Liouville mode. (This is most obvious in a path integral formulation.) The resulting theory is much more complicated then the critical string, because its world-sheet theory is interacting even for a flat target space. For this theory much less is known then about the critical string. However, there are arguments indicating that the non-critical string is equivalent to the critical string in a non-trivial background.

We will only consider critical string theories in the following. Also note that the above analysis applies to strings in flat space-time, with no background fields. When switching on a non-trivial dilaton background, this can modify the central charge of the world-sheet conformal field theory, and, hence, the dimension of space-time. But this topic is beyond the scope of these lectures.

The spectrum of the bosonic closed string. We can now identify the physical states by imposing the constraints. Let us consider closed strings. We first look at the two constraints

$$
\left(L_{0}-1\right)|\Phi\rangle=0, \quad\left(\tilde{L}_{0}-1\right)|\Phi\rangle=0 .
$$

The operator $L_{0}$ can be rewritten as

$$
L_{0}=\frac{1}{8} p^{2}+N .
$$


As mentioned above the operator $L_{0}$ is the normal ordered version of (29) with $m=0$. The original and the normal ordered expression formally differ by an infinite constant. Subtracting this constant introduces a finite ambiguity, which was parametrized by $a$. Unitarity then fixed $a=1$. The oscillator part of $L_{0}$ is

$$
N=\sum_{n=1}^{\infty} \alpha_{-n} \cdot \alpha_{n}
$$

$N$ is called the number operator, because

$$
\left[N, \alpha_{-m}^{\mu}\right]=m \alpha_{-m}^{\mu} .
$$

Since the total momentum is related to the mass of the string by $M^{2}+p^{2}=0$, the constraints (37) determine the mass of a physical states in terms of the eigenvalues of $N$ and of its right-moving analogue $\tilde{N}$. (We denote the operators and their eigenvalues by the same symbol.) We now use the above decomposition of $L_{0}$, take the sum and difference of the constraints (37) and reintrodue the Regge slope $\alpha^{\prime}=\frac{1}{2}$ by dimensional analysis:

$$
\begin{aligned}
& \alpha^{\prime} M^{2}=2(N+\tilde{N}-2) \\
& N \quad=\tilde{N}
\end{aligned}
$$

The first equation is the mass formula for string states, wherea the second equation shows that left- and right-moving degrees of freedom must constribute equally to the mass.

Let us list the lightest states satisfying these constraints:

\begin{tabular}{|l|l|l|}
\hline Occupation & Mass & State \\
\hline \hline$N=\tilde{N}=0$ & $\alpha^{\prime} M^{2}=-4$ & $|k\rangle$ \\
\hline$N=\tilde{N}=1$ & $\alpha^{\prime} M^{2}=0$ & $\alpha_{-1}^{\mu} \tilde{\alpha}_{-1}^{\nu}|k\rangle$ \\
\hline$N=\tilde{N}=2$ & $\alpha^{\prime} M^{2}=4$ & $\alpha_{-2}^{\mu} \tilde{\alpha}_{-2}^{\nu}|k\rangle$ \\
& & $\alpha_{-2}^{\mu} \tilde{\alpha}_{-1}^{\nu} \tilde{\alpha}_{-1}^{\rho}|k\rangle$ \\
& & $\alpha_{-1}^{\mu} \alpha_{-1}^{\nu} \tilde{\alpha}_{-2}^{\rho}|k\rangle$ \\
& & $\alpha_{-1}^{\mu} \alpha_{-1}^{\nu} \tilde{\alpha}_{-1}^{\rho} \tilde{\alpha}_{-1}^{\sigma}|k\rangle$ \\
\hline
\end{tabular}

The most obvious and disturbing fact is that the ground state is a tachyon, i.e., a state of negative mass squared. Since the mass squared of a scalar corresponds to the curvature of the potential at the critical point, we seem to be expanding around a maximum rather then a minimum of the potential. This signals that the bosonic closed string quantized in flat Minkowski space is unstable. It is a very interesting question whether there is a minimum of this potential which provides a stable ground state. Since the tachyon aquires a vacuum expectation value in this minimum, this is referred to as tachyon condensation. 
But since we will be mostly interested in superstring theories, where tachyons are absent, we will simply ignore the fact that our toy model has a tachyon.

The first excited state is massless, and on top of it we find an infinite tower of states with increasing mass. Since the mass scale of string theory presumably is very large, we will focus on the massless states. So far we only imposed the constraints (37). The other constraints

$$
L_{m}|\Phi\rangle=0, \quad \tilde{L}_{m}|\Phi\rangle=0, \quad m>0,
$$

impose conditions on the polarisations of physical states. For the tachyon one gets no condition, while for the first excited level the constraints with $m=1$ are non-trivial. Forming a general linear combination of basic states,

$$
\zeta_{\mu \nu} \alpha_{-1}^{\mu} \tilde{\alpha}_{-1}^{\nu}|k\rangle
$$

the constraints $(43)$ imply

$$
k^{\mu} \zeta_{\mu \nu}=0=k^{\nu} \zeta_{\mu \nu} .
$$

Since $\zeta_{\mu \nu}$ is the polarization tensor, we see that only states of transverse polarization are physical. To obtain the particle content, we have to extract the irreducible representations of the D-dimensional Poincaré group contained in physical $\zeta_{\mu \nu}$. There are three such representations: the traceless symmetric part describes a graviton $G_{\mu \nu}$, the trace part corresponds to a scalar, the dilaton $\Phi$, and the third representation is an antisymmetric tensor $B_{\mu \nu}$. In order to disentangle the trace part, one needs to introduce an auxiliary vector $\bar{k}$, with the properties:

$$
\bar{k} \cdot \bar{k}=0, \quad k \cdot \bar{k}=-1 .
$$

( $k$ is the momentum vector.) The polarization tensors of the graviton, dilaton and antisymmmetric tensor are:

$$
\begin{aligned}
\zeta_{\mu \nu}^{G} & =\zeta_{(\mu \nu)}-\frac{1}{D-2} \zeta_{\rho}^{\rho}\left(\eta_{\mu \nu}-k_{\mu} \bar{k}_{\nu}-k_{\nu} \bar{k}_{\mu}\right), \\
\zeta_{\mu \nu}^{\Phi} & =\frac{1}{D-2} \zeta_{\rho}^{\rho}\left(\eta_{\mu \nu}-k_{\mu} \bar{k}_{\nu}-k_{\nu} \bar{k}_{\mu}\right), \\
\zeta_{\mu \nu}^{B} & =\zeta_{[\mu \nu]},
\end{aligned}
$$

where $\zeta_{(\mu \nu)}=\frac{1}{2}\left(\zeta_{\mu \nu}+\zeta_{\nu \mu}\right)$ and $\zeta_{[\mu \nu]}=\frac{1}{2}\left(\zeta_{\mu \nu}-\zeta_{\nu \mu}\right)$ are the symmetric and antisymmetric parts of $\zeta_{\mu \nu}$. Note that the prefactor $1 /(D-2)$ is needed in order that the trace part is physical. Using explicit choices for $k, \bar{k}$ one can check that $\zeta_{\mu \nu}^{G}$ is the polarization tensor of a plane wave and transforms as a traceless symmetric tensor under transverse rotations.

As we discussed above, physical states are only defined up to the addition of null states, $|\Phi\rangle \sim|\Phi\rangle+|\Psi\rangle$. In the case at hand adding null states corresponds to adding states of longitudinal polarization, according to:

$$
\begin{aligned}
\zeta_{(\mu \nu)} & \sim \zeta_{(\mu \nu)}+k_{\mu} \zeta_{\nu}+\zeta_{\mu} k_{\nu} \\
\zeta_{[\mu \nu]} & \sim \zeta_{[\mu \nu]}+k_{\mu} \xi_{\nu}-\xi_{\mu} k_{\nu} .
\end{aligned}
$$


$\zeta_{\mu}$ and $\xi_{\mu}$ are arbitrary vectors orthogonal to the momentum $k^{\mu}$. Adding null states can be understood as a residual gauge transformation parametrized by $\zeta_{\mu}, \xi_{\mu}$. By taking Fourier transforms we see that these are the standard gauge invariances of a graviton and of an antisymmetric tensor, repectively:

$$
\begin{aligned}
G_{\mu \nu} & \sim G_{\mu \nu}+\partial_{\mu} \Lambda_{\nu}+\partial_{\nu} \Lambda_{\mu}, \\
B_{\mu \nu} & \sim B_{\mu \nu}+\partial_{\mu} A_{\nu}-\partial_{\nu} A_{\mu} .
\end{aligned}
$$

A graviton is defined by taking the gravitational action and expanding the metric around a flat background. The gauge transformations are then infinitesimal reparametrization, which, in a flat background, act according to (49) on the metric. Note that our gauge transformations $\Lambda_{\mu}, A_{\mu}$ have a vanishing divergence, because the corresponding polarization vectors are orthogonal to the momentum. This reason is that the Virasoro constraints automatically impose a generalized Lorentz gauge.

Thus far our identification of the symmetric traceless part of the state (44) as a graviton is based on the fact that this state has the same kinematic properties as a graviton in Einstein gravity. We will see later, after analyzing string interactions, that this extends to the dynamical properties.

Finally it is interesting to compare the results of old covariant quantization to those obtained in light cone quantization. In light cone quantization unitarity is manifest, but the Lorentz algebra of the quantum theory has an anomaly which only cancels in the critical dimension $D=26$. Moreover the normal ordering constant must take the value $a=1$. Independently, the same value of $a$ is obtained when computing the Casimir energy of the ground state using $\zeta$-function regularization. One virtue of light cone quantization is that one can write down immediately all the physical states. A basis is provided by all states which can be created using transverse oscillators,

$$
\alpha_{-m_{1}}^{i_{1}} \cdots \tilde{\alpha}_{-n_{1}}^{j_{1}} \cdots|k\rangle,
$$

where $i_{1}, \ldots, j_{1}, \ldots=1, \ldots, D-2$. What remains is to group these states into representations of the D-dimensional Poincaré group. Massless states are classified by the little group $S O(D-2)$. Since all states manifestly are tensors with respect to this subgroup, one immediately sees that the massless states are a graviton (traceless symmetric tensor), dilaton (trace) and antisymmetric tensor. For massive states the little group is the full rotation subgroup $S O(D-1)$. Using Young tableaux it is straightforward to obtains these from the given representations of $S O(D-2)$.

Open strings. Having treated the closed bosonic string in much detail, we now describe the results for open strings. One finds the same critical dimension, $D=26$, and the same value of the normal ordering constant, $a=1$. The constraints read:

$$
\left(L_{0}-1\right)|\Phi\rangle=0, \quad L_{m}|\Phi\rangle=0, \quad m>0 .
$$


$L_{0}$ can be decomposed as $L_{0}=\frac{1}{2} p^{2}+N$, where $N$ is the number operator. The $L_{0}$-constraint gives the mass formula:

$$
\alpha^{\prime} M^{2}=N-1 .
$$

Therefore the lowest states are:

\begin{tabular}{|l|l|l|}
\hline Occupation & Mass & State \\
\hline \hline$N=0$ & $\alpha^{\prime} M^{2}=-1$ & $|k\rangle$ \\
\hline$N=1$ & $\alpha^{\prime} M^{2}=0$ & $\zeta_{\mu} \alpha_{-1}^{\mu}|k\rangle$ \\
\hline$N=2$ & $\alpha^{\prime} M^{2}=1$ & $\begin{array}{l}\zeta_{\mu \nu} \alpha_{-1}^{\mu} \alpha_{-1}^{\nu}|k\rangle \\
\zeta_{\mu} \alpha_{-2}^{\mu}|k\rangle\end{array}$ \\
\hline
\end{tabular}

The other constraints impose restrictions on the polarizations. Whereas the groundstate is a tachyonic scalar, the massless state has the kinematic properties of a gauge boson: its polarization must be transverse,

$$
\zeta_{\mu} k^{\mu}=0,
$$

and polarizations proportional to $k^{\mu}$ correspond to null states,

$$
\zeta_{\mu} \sim \zeta_{\mu}+\alpha k_{\mu} .
$$

This is the Fourier transform of a $U(1)$ gauge transformation,

$$
A_{\mu} \sim A_{\mu}+\partial_{\mu} \chi .
$$

Whereas massless closed string states mediate gravity, massless open string states mediate gauge interactions.

Chan-Paton factors. Open string theory has a generalization which has nonabelian gauge interactions. One can assign additional degress of freedom to the ends of the string, namely charges ('Chan-Paton factors') which transform in the fundamental and anti-fundamental (complex conjugated) representation of the group $U(n)$. The massless states then take the form

$$
\zeta_{\mu} \alpha_{-1}^{\mu}|k, a, \bar{b}\rangle,
$$

where $a$ is an index transforming in the fundamental representation $[n]$ of $U(n)$, whereas $\bar{b}$ transforms in the anti-fundamental representation $[\bar{n}]$. Since

$$
[n] \times[\bar{n}]=\operatorname{adj} U(n),
$$

the massless states transform in the adjoint of $U(n)$ and can be interpreted as $U(n)$ gauge bosons. (As for the graviton, we have only seen the required kinematic properties so far. But the interpretation is confirmed when studying interactions.)

Note that $U(n)$ is the only compact Lie group where the adjoint representation is the product of the fundamental and anti-fundamental representation. Therefore the construction precisely works for these groups. 
Non-oriented strings. There is a further modification which leads to nonoriented strings. These are obtained from the theories constructed so far by a projection. Both closed and open bosonic string theories are symmetric under world-sheet parity, which is defined as a reflection on the world-sheet:

$$
\Omega: \sigma^{1} \longrightarrow \pi-\sigma^{1}=-\sigma^{1} \text { modulo } \pi \text {. }
$$

Since $\Omega$ is an involution, $\Omega^{2}=1$, the spectrum can be organized into states with eigenvalues \pm 1 :

$$
\begin{aligned}
& \Omega|N, k\rangle=(-1)^{N}|N, k\rangle, \\
& \Omega|N, \tilde{N}, k\rangle=|\tilde{N}, N, k\rangle .
\end{aligned}
$$

Here $|N, k\rangle$ is an open string state with momentum $k$ and total occupation number $N$ and $|N, \tilde{N}, k\rangle$ is a closed string state with momentum $k$ and total left and right occupation numbers $N, \tilde{N}$.

Non-oriented strings are defined by keeping only those states which are invariant under $\Omega$. The resulting theories are insensitive to the orientation of the world-sheet. Let us look at the effect of this projection on the lowest states. For open strings we are left with:

\begin{tabular}{|l|l|l|}
\hline Occupation & Mass & State \\
\hline \hline$N=0$ & $\alpha^{\prime} M^{2}=-1$ & $|k\rangle$ \\
\hline$N=1$ & $\alpha^{\prime} M^{2}=0$ & - \\
\hline$N=2$ & $\alpha^{\prime} M^{2}=1$ & $\begin{array}{l}\zeta_{\mu \nu} \alpha_{-1}^{\mu} \alpha_{-1}^{\nu}|k\rangle \\
\zeta_{\mu} \alpha_{-2}^{\mu}|k\rangle\end{array}$ \\
\hline
\end{tabular}

All states with odd occupation numbers are projected out, including the gauge boson. For closed strings we obtain:

\begin{tabular}{|l|l|l|}
\hline Occupation & Mass & State \\
\hline \hline$N=\tilde{N}=0$ & $\alpha^{\prime} M^{2}=-4$ & $|k\rangle$ \\
\hline$N=\tilde{N}=1$ & $\alpha^{\prime} M^{2}=0$ & $\zeta_{(\mu \nu)} \alpha_{-1}^{\mu} \tilde{\alpha}_{-1}^{\nu}|k\rangle$ \\
\hline$N=\tilde{N}=2$ & $\alpha^{\prime} M^{2}=4$ & $\begin{array}{l}\zeta_{(\mu \nu)} \alpha_{-2}^{\mu} \tilde{\alpha}_{-2}^{\nu}|k\rangle \\
\zeta_{(\mu \rho \nu \sigma)} \alpha_{-1}^{\mu} \alpha_{-1}^{\nu} \tilde{\alpha}_{-1}^{\rho} \tilde{\alpha}_{-1}^{\sigma}|k\rangle\end{array}$ \\
\hline
\end{tabular}

Only states which are left-right symmetric survive. At the massless level the antisymmetric tensor is projected out, whereas the graviton and dilaton are kept.

Chan-Paton factors for non-oriented strings. The above construction can be generalized to open strings with Chan-Paton factors. In this case the two 
representations assigned to the ends of the strings must be equivalent. One can define a generalized involution $\Omega^{\prime}$, which combines world-sheet parity with an action on the Chan-Paton indices,

$$
\Omega^{\prime}|N, a, b\rangle=\varepsilon(-1)^{N}|N, b, a\rangle,
$$

where $\varepsilon= \pm 1$. The projection is $\Omega^{\prime}|N, a, b\rangle \stackrel{!}{=}|N, a, b\rangle$. There are two inequivalent choices of the projection. For $\varepsilon=1$, the indices $a, b$ must transform in the fundamental representation of $S O(n)$. Since the adjoint of $S O(n)$ is the antisymmetric product of the fundamental representation with itself, the massless vector state transforms in the adjoint. More generally, states at even (odd) mass level transform as symmetric (antisymmetric) tensors.

The other choice is $\varepsilon=-1$. Then $a, b$ transform in the fundamental of $U S p(2 n)$ (the compact form of the symplectic group. Our normalization is such that $U S p(2) \simeq S U(2)$.) Since the adjoint of $U S p(2 n)$ is the symmetric product of the fundamental representation with itself, the massless vector transforms in the adjoint. More generally, states at even (odd) mass level transform as antisymmetric (symmetric) tensors.

D-branes. Finally we can consider open strings with Dirichlet boundary conditions along some directions. Consider first oriented open strings ending on a D-p-brane located at $x_{(1)}^{a}$. The ground state is tachyonic. The massless state of an open string with purely Neumann condition is a D-dimensional gauge boson $\alpha_{-1}^{\mu}|k\rangle$. Now we impose Dirichlet boundary conditions along the directions $a=p+1, \ldots, D-1$, so that the string can only move freely along the Neumann directions $m=0,1, \ldots, p$. The relevant kinematic group is now the world-volume Lorentz group $S O(1, \mathrm{p})$. The massless states are a world-volume vector,

$$
\alpha_{-1}^{m}|k\rangle, \quad m=0,1, \ldots, \mathrm{p}
$$

and $\mathrm{D}-\mathrm{p}-1$ scalars

$$
\alpha_{-1}^{a}|k\rangle, \quad a=\mathrm{p}+1, \ldots, \mathrm{D}-1 .
$$

The scalars correspond to transverse oscillations of the brane. Changing the position of the brane corresponds to changing the vacuum expectation values of the scalars. The effective action of the massless modes is given, to leading order in $\alpha^{\prime}$, by the dimensional reduction of the D-dimensional Maxwell action to $\mathrm{p}+1$ dimensions. The full effective action is of Born-Infeld type.

Next consider $N$ parallel D-p-branes, located at positions $x_{(i)}^{a}$. The new feature of this configuration is that there are strings which start and end on different branes. For such strings there is an additional term in the mass formula, which accounts for the stretching:

$$
\alpha^{\prime} M^{2}=N-1+\left(\frac{\left|\boldsymbol{x}_{(i)}-\boldsymbol{x}_{(j)}\right|}{2 \pi \sqrt{\alpha^{\prime}}}\right)^{2} .
$$


Here $\boldsymbol{x}_{(i)}$ is the position of the i-th brane. (Remember that the tension of the string is $\left(2 \pi \alpha^{\prime}\right)^{-1}$.) Due to the normal ordering constant, the ground state becomes tachyonic if two branes come close enough. This signals an instability of the D-brane configuration. As already mentioned this might lead to interesting dynamics (tachyon condensation, decay of D-branes), but we will not discuss this here. Instead, we focus on features shared by D-branes in supersymmetric string theories. The states at the first excited level become massless precisely if the corresponding D-branes are put on top of each other. Each of the $N$ branes already carries a $U(1)$ gauge theory: the massless modes of strings beginning and ending of the same brane give $N$ vectors and $N \cdot(\mathrm{D}-\mathrm{p}-1)$ scalars. For $N$ coinciding branes we get additional $N \cdot(N-1)$ vectors and $N \cdot(N-1) \cdot(\mathrm{D}-\mathrm{p}-1)$ scalars. Combining all massless states one gets one vector and $\mathrm{D}-\mathrm{p}-1$ scalars in the adjoint representation of the non-abelian group $U(N)$. This suggests that the D-brane system describes a $U(N)$ gauge theory with an adjoint Higgs mechanism. The Higgs mechanism is realized geometrically: Higgs expectation values correspond to the distances between branes, and the masses can be understood in terms of stretched strings. Again, this interpretation, which is based on analyzing the spectrum is confirmed when studying interactions. Besides Chan-Paton factors, D-branes are a second possibility to introduce non-abelian gauge groups. In fact Chan-Paton factors are related to D-branes through T-duality, but we will not be able to discuss this in these lectures.

The above construction can be extended to non-oriented strings, where other gauge groups occure. There are various other generalizations, which allow one to construct and study various gauge theories using strings and D-branes. These techniques are known as 'D-brane engineering' of field theories. Besides being of interest for the study of field theories through string methods, D-branes are important for understanding string theory itself. As we will see later, D-branes are actually solitons of string theory. Thus we are in the privileged position of knowing the exact excitation spectrum around such solitons in terms of open strings. This can be used, for example, to compute the entropy and Hawking radiation of black holes.

Another application of D-branes goes under the name of 'brane worlds' or 'brane universes' or 'models with large extra dimensions'. As we have seen, Dbranes enable one to localize gauge interactions and matter on a lower-dimensional submanifold of space-time. This leads to models with space-dimensions where only gravity (closed strings) but not standard model matter (open strings) can propagate. Empirical limits on the size of the dimensions transverse to the brane only come from gravity, which is much weaker than all other interactions. Therefore such dimensions can be quite large, even up to about $1 \mathrm{~mm}$. This is in contrast to limits on extra dimensions which are accessible to standard model interactions. Here the experimental limits are set by the scale resolvable in current accelerator experiments.

Brane world models are nowadays popular in both particle physics and cosmology. In particular, they can be used to construct models where the fundamen- 
tal gravitational scale is of order $1 \mathrm{TeV}$. We will come back to these applications of D-branes in section 6.

\subsection{Further reading}

The material covered in this section can be found in all of the standard textbooks on string theory [1, 2, 3, 3,, 5 . Dirichlet boundary conditions and D-branes are only covered by the more recent ones [3, 1 .

\section{$3 \quad$ Interacting bosonic strings}

So far we have not specified how strings interact. One might expect that this can be done by adding interaction terms to the world-sheet action. However, we have to respect the local symmetries of the Polyakov action, which severely restricts our options. In particular, contact interactions, which are frequently used in describing non-fundamental string-like objects such as polymers, are not compatible with Weyl invariance. Admissible interacting world-sheet actions include marginal deformations of the Polyakov action, i.e., deformations which preserve Weyl invariance. One such deformation replaces the flat space-time metric by a curved one. As expected intuitively, such an action does not describe interactions among strings, but strings moving in a non-trivial background. The same is true when replacing the Polaykov action by more general conformal field theories.

How then do we define interactions? We will give a heuristic discussion in the next section. The resulting scattering amplitudes are Lorentz covariant, unitary and UV finite. They include the Veneziano amplitude and its cousins, which historically started the subject.

For definiteness we will focus in the following on closed oriented strings. The generalization to other string theories will be indicated briefly.

\subsection{Heuristic discussion}

Intuitively, interactions between strings are described by world-sheets which connect a given initial configuration of strings to a final configuration. One can draw several such world-sheets, which differ by their topologies. Comparing to the similar treatment of point particles by graphs, we realize that while graphs have vertices, the world-sheets connecting strings are manifolds without distinguished interaction points. This leads to the expectation that string interactions are less singular then those of point particles, which is indeed confirmed by the final result of the construction. Moreover, it indicates that one does not have any freedom in defining interactions. For particles, we can assign couplings to vertices which depend on the species of the particles meeting at the vertex. For strings the interaction is encoded in the topology of the world-sheet and there is no such freedom. There is one fundamental interaction, which couples three closed strings, and all we can do is to assign a coupling constant $\kappa$ to it. 
Next, we restrict ourselves to finding transition amplitudes between asymptotic states in the infinite past and future. An asymptoting in- or out-going state is represented by a semi-infinite cylinder. When mapping this to a punctered disc, the asymptotic state is represented by the puncture. This leads to the idea that we can represent the asymptotic state by a local operator of the world-sheet field theory. Such operators are called vertex operators. Note that they do not describe interactions. Instead, the vertex operator $V_{\Phi}(\sigma)$ describes the creation or annihilation of the string state $|\Phi\rangle$ at the position $\sigma$ on the world-sheet. That is, they allow us to assign a copy of the space of physical states to every point of the world-sheet. As we will see below, there is indeed a natural one-to-one map between physical states $|\Phi\rangle$ and local operators of the world-sheet field theory.

After replacing the world-sheet punctures by insertions of vertex operators we are left with compact closed surfaces. The topologies of such surfaces are classified by their genus $g \geq 0$, or equivalently, by their Euler number $\chi=2-2 g$. Here $g=0$ is the two-sphere, and $g=1$ is the torus. The general genus $g$ surface $\Sigma_{g}$ is obtained from the sphere by attatching $g$ handles. The handles play the role of loops in Feynman diagrams. When considering an interaction process on $\Sigma_{g}$ involving $M$ external states, we find $M-\chi$ fundamental string interactions and have to assign a factor $\kappa^{M-\chi}$.

We now postulate that a scattering amplitude involving $M$ external states is given by

$$
A(1, \ldots, M)=\sum_{g=0}^{\infty} \kappa^{M-\chi} A(1, \ldots, M)_{g},
$$

where $A(1, \ldots, M)_{g}$ is the contribution of $\Sigma_{g}$. This is a perturbative expression in the string coupling $\kappa$. As usual for theories with a single coupling, the expansion in the coupling coincides with the expansion in loops, which in our case is the expansion in the genus $g$.

The genus $g$ contribution is defined to be

$$
A(1, \ldots, M)_{g}=\left\langle V_{1} \cdots V_{M}\right\rangle_{g},
$$

where

$$
V_{i}=\int_{\Sigma_{g}} d^{2} \sigma_{i} \sqrt{h} V_{i}\left(\sigma_{i}\right)
$$

are the so-called integrated vertex operators, which are obtained by integrating the vertex operators $V_{i}\left(\sigma_{i}\right)$ over the world sheet. (Though our notation might suggest otherwise, we do not require that $\Sigma_{g}$ can be covered by one set of coordinates, which is of course impossible for compact $\Sigma_{g}$. We just use a local representative of the integrand for notational purposes.) In (69) we compute the correlation function of the vertex operators $V_{i}\left(\sigma_{i}\right)$ on $\Sigma_{g}$ in the world-sheet quantum field theory defined by the Polyakov action and integrate over the positions of the vertex operators. The result is interpreted as a scattering amplitude of string states in space-time, with the in- and out-states represented by the vertex operators. 
Note that it is not possible to introduce arbitrary weight factors between the contributions of different genera. The reason is that unitarity requires that scattering amplitudes factorize into the amplitudes of subprocesses whenever an intermediate state is on-shell. In fact, in the old days of string theory this was used to construct the perturbative expansion by seewing together tree amplitudes. However, this approach is more cumbersome then the Polyakov path integral approach that we will desribe here.

\section{$3.2 \quad$ Vertex operators}

We now take a closer look at the vertex operators. Observe that the scattering amplitudes defined by $68,69,70)$ must be invariant under reparametrizations of the world-sheets. In particular the local vertex operators $V_{i}\left(\sigma_{i}\right)$ must transform such that $(70)$ is invariant. When imposing the conformal gauge, it still must transform in a specific way under conformal transformations $\sigma^{ \pm} \rightarrow \tilde{\sigma}^{ \pm}\left(\sigma^{ \pm}\right)$. In conformal field theory fields which transform covariantly under conformal transformations are called primary conformal fields. A primary conformal field of weights $(h, \bar{h})$ is an object that transforms like a contravariant tensor field of $\operatorname{rank}(h, \bar{h})$ :

$$
\tilde{V}\left(\tilde{\sigma}^{+}, \tilde{\sigma}^{-}\right)=\left(\frac{d \sigma^{+}}{d \tilde{\sigma}^{+}}\right)^{h}\left(\frac{d \sigma^{-}}{d \tilde{\sigma}^{-}}\right)^{\bar{h}} V\left(\sigma^{+}, \sigma^{-}\right) .
$$

Invariance of (70) implies that vertex operators of physical states must be primary conformal fields of weights $(1,1)$. This property is equivalent to imposing the Virasoro constraints (41) on physical states. States assigned to a point $P$ of $\Sigma$ are constructed from vertex operators by applying them to a ground state $|0\rangle_{P}$

$$
|\Phi\rangle=V_{\Phi}(P)|0\rangle_{P}
$$

To make contact with the space $\mathcal{F}_{\text {phys }}$ constructed in section 2.2, one parametrizes $\Sigma$ in the vicinity of $P$ by a semi-infinity cylinder with $P$ being the asymptotic point $\sigma^{0} \rightarrow-\infty$. Intuitively this describes an ingoing state created in the infinite past. Then,

$$
|\Phi\rangle=\lim _{\sigma^{0} \rightarrow-\infty} V_{\Phi}(\sigma)|0\rangle
$$

where $|0\rangle:=|k=0\rangle$ is the (unphysical) zero-momentum state with occupation numbers $N=0=\tilde{N}$ in $\mathcal{F}$.

To indicate how this works in practice, we now specify the vertex operators for the lowest states. Consider the operator

$$
V(\sigma)=: e^{\mathrm{i} k_{\mu} X^{\mu}}:(\sigma)
$$

where $: \cdots$ : indicates normal ordering. Applying this operator we find

$$
\lim _{\sigma^{0} \rightarrow-\infty}: e^{\mathrm{i} k_{\mu} X^{\mu}}:(\sigma)|0\rangle=e^{\mathrm{i} k_{\mu} x^{\mu}}|0\rangle=|k\rangle,
$$

where we used that $e^{\mathrm{i} k_{\mu} x^{\mu}}|0\rangle$ is an eigenstate of $p^{\mu}$ with eigenvalues $k^{\mu}$. One can show that (74) has weights $\left(\frac{1}{8} k^{2}, \frac{1}{8} k^{2}\right)$. Thus it has weights $(1,1)$ if $k^{2}=8$, which 
is the physical state condition $M^{2}=-8$ for the tachyonic ground state of the closed string. (We have set $\alpha^{\prime}=\frac{1}{2}$.)

The vertex operator for the first excited level is

$$
V(\sigma)=: \zeta_{\mu \nu} \partial_{+} X^{\mu} \partial_{-} X^{\nu} e^{\mathrm{i} k_{\rho} X^{\rho}}:(\sigma) .
$$

This has weights $(1,1)$ if

$$
k^{2}=0, \quad k^{\mu} \zeta_{\mu \nu}=0=k^{\nu} \zeta_{\mu \nu},
$$

which is precisely the physical state condition for the state

$$
\zeta_{\mu \nu} \alpha_{-1}^{\mu} \tilde{\alpha}_{-1}^{\nu}|k\rangle \text {. }
$$

More generally, vertex operators of the form

$$
V(\sigma)=: \zeta_{\mu_{1} \cdots \nu_{1} \cdots} \partial_{+}^{m_{1}} X^{\mu_{1}} \cdots \partial_{-}^{n_{1}} X^{\nu_{1}} \cdots e^{\mathrm{i} k_{\rho} X^{\rho}}:(\sigma)
$$

generate states of the form

$$
\zeta_{\mu_{1} \cdots \nu_{1} \cdots} \alpha_{-m_{1}}^{\mu_{1}} \cdots \tilde{\alpha}_{-n_{1}}^{\nu_{1}} \cdots|k\rangle .
$$

\subsection{Interactions in the path integral formalism}

The next step is to explain in more detail how the amplitudes (68)-(70) are defined and how they are computed in practice. As usual one can use either the path integral (Lagrangian) or the operator (Hamiltonian) formulation. We will use Polyakov's path integral formulation. This has the advantage of immediately providing explicit formal expressions for correlation functions. The mathematical complications of defining the interacting quantum theory are hidden in the path integral measure. We will not discuss this is full detail, but mention and illustrate the most important points.

The path integral. We now turn to the Polaykov path integral, which is one way to give a precise meaning to (68). In this approach the correlation function $(69)$ is computed by functional methods. Intuitively we integrate over all paths that strings can take in space-time. However, in order to have a well defined path integral, we need to study the theory in Euclidean signature, both on the world-sheet and in space-time. A Euclidean formulation of the world-sheet theory is needed to have a well defined functional integral for the world-sheet field theory. In particular, we want to have well defined world-sheet metrics on general surfaces $\Sigma_{g}$, which is not possible for Lorentzian signature. Second, one also has to work in Euclidean space-time, in order to have a standard Gaussian integral for the 'time' coordinate $X^{0}$. Wick-rotating $X^{0}$ can be interpreted as continuing to unphysical Euclidean momenta and polarizations. As we have seen in our discussion of vertex operators the string coordinates $X^{\mu}$ are always contracted with momenta and polarizations. Physical scattering amplitudes are 
thus obtained by computing (68) in the Euclidean theory and evaluating the result for physical momenta and polarizations. This uses the analycity properties expected to hold for any relativistic unitary scattering amplitude. For tree-level amplitudes one can study how the Wick rotation works explicitly, by comparing to results obtained by operator methods.

Our starting point is the Polyakov action on a world-sheet $\Sigma$ with positive definite metric $h_{\alpha \beta}$ and local complex coordinate $z$,

$$
S_{\mathrm{P}}=\frac{1}{4 \pi \alpha^{\prime}} \int_{\Sigma} d^{2} z \sqrt{h} h^{\alpha \beta} \partial_{\alpha} X^{\mu} \partial_{\beta} X_{\mu} .
$$

The quantum theory is now defined by summing over all topologies of $\Sigma$ and integrating over $X^{\mu}$ and $h_{\alpha \beta}$ :

$$
A(1, \ldots, M)=\sum_{g=0}^{\infty} \kappa^{M-\chi} N_{g} \int D X^{\mu} D h_{\alpha \beta} e^{-S_{P}[X, h]} V_{1} \cdots V_{M}
$$

where $V_{i}$ are the integrated vertex operators of the physical states and $N_{g}$ are normalization factors needed to define the path integral. The $V_{i}$ depend on $X^{\mu}$ through the local vertex operators $V_{i}\left(\sigma_{i}\right)$, while the world-sheet metric enters through the integration over $\sigma_{i}$.

One expects that one can properly define and compute the expression (82), because the integration over $X^{\mu}$ is Gaussian (in flat space-time) and $h_{\alpha \beta}$ is nondynamical. This turns out to be true, though several interesting complications arise. Let us consider the integration over $h_{\alpha \beta}$. Since we can locally impose the conformal gauge,

$$
h_{\alpha \beta}=\delta_{\alpha \beta},
$$

we expect that we can use the Faddeev-Popov method and trade the integration over the metric for an integration over reparametrizations and the Weyl factor. As long as these are symmetries, the corresponding integration factorizes and can be absorbed in the normalization factor $N_{g}$. The first obstruction encountered is that there is a conformal anomaly when the quantum theory based on (81) lives on a curved world-sheet. This has the consequence that the integration over the Weyl factor does not factorize in general. One option is to accept it as a new, purely quantum degree of freedom: this is non-critical string theory, also called Liouville string theory, because the dynamcis of the Weyl factor is given by the Liouville action. The other option is to observe that the anomaly is proportional to $D-26$, and therefore cancels for $D=26$ space-time dimensions. This is the critical string theory we study in these lectures.

Moduli and modular transformations. The next point is that the gauge (83) cannot be imposed globally. All that can be achieved is to map $h_{\alpha \beta}$ to a metric of constant curvature,

$$
h_{\alpha \beta} \stackrel{!}{=} \hat{h}_{\alpha \beta}[\boldsymbol{\tau}] .
$$


As indicated, $\Sigma_{g}$ in general posesses a continuous family of such metrics, parametrized by moduli $\boldsymbol{\tau}=\left(\tau_{1}, \ldots\right)$. The space of constant curvature metrics on a two-dimensional closed compact surface is isomorphic to the space of complex structures. By reparametrizations and Weyl transformations we cannot change the complex structure of the metric but we can map it to the unique representative (84) of the complex structure class which has constant curvature. Then the path integral over all metrics reduces to a finite-dimensional integral over the space $\mathcal{M}_{g}$ of complex structures. The dimension of this space is known from a Riemann-Roch theorem. For $g=0$ the complex structure is unique, and every metric can be mapped to the standard round metric on the sphere. For $g>1$ there is a non-trivial moduli space,

$$
\begin{aligned}
\operatorname{dim}_{\mathbf{C}} \mathcal{M}_{g} & =1, \quad \text { for } g=1, \\
\operatorname{dim}_{\mathbf{C}} \mathcal{M}_{g} & =3 g-3, \quad \text { for } g>1 .
\end{aligned}
$$

After carrying out the integration over the metric, amplitudes take the form

$$
A(1, \ldots, M)=\sum_{g=0}^{\infty} \kappa^{M-\chi} N_{g}^{\prime} \int_{\mathcal{M}_{g}} d \mu(\boldsymbol{\tau}) \int D X^{\mu} e^{-S_{P}[X, \hat{h}]} J(\hat{h}) V_{1} \ldots V_{M} .
$$

$N_{g}^{\prime}$ are normalization factors needed to deal with the $X^{\mu}$-integration and $J(\hat{h})$ is the Faddeev-Popov determinant, which one can rewrite as a functional integral over Faddeev-Popov ghost fields. As indicated the $X^{\mu}$-integral depends on the moduli through the world-sheet metric $\hat{h}_{\alpha \beta}=\hat{h}_{\alpha \beta}(\tau)$. One finds that the measure $d \mu(\boldsymbol{\tau})$ for the moduli is the natural measure on the space of complex structures, the so-called Weil-Petersson measure.

The precise characterization of the moduli space has further interesting details. We examplify this with the two-torus. We can represent a torus as a parallelogram in the complex plane with opposite sides identified. Since the complex structure does not depend on the overall volume, we can restrict ourselves to parallelograms with edges $0,1, \tau, \tau+1$, where $\operatorname{Im}(\tau)>0$. In one complex dimension holomorphic maps are conformal maps, and vice versa. Thus the complex structure is varied by moving $\tau$ in the upper half-plane,

$$
\mathcal{H}=\{\tau \in \mathbf{C} \mid \operatorname{Im}(\tau)>0\} .
$$

This is the modulus we are looking for. $\mathcal{H}$ has a metric of constant negative curvature, the Poincaré metric,

$$
d \mu(\tau)=\frac{d^{2} \tau}{(\operatorname{Im}(\tau))^{2}} .
$$

With this $S L(2, \mathbf{R})$-invariant metric, $\mathcal{H}$ is the symmetric space $S l(2, \mathbf{R}) / S O(2)$. However, $\mathcal{H}$ is not our moduli space, because it overcounts complex structures. On $\mathcal{H}$ the group $S l(2, \mathbf{R})$ acts from the right. Taking $\tau$ as coordinate, the operation is

$$
\tau \longrightarrow \frac{a \tau+b}{c \tau+d}, \quad \text { where }\left(\begin{array}{l}
a b \\
c d
\end{array}\right) \in \operatorname{Sl}(2, \mathbf{R}) .
$$


The subgroup $\operatorname{Sl}(2, \mathbf{Z})$ maps parallelograms to parallelograms which define the same torus, because they form basic cells of the same lattice in $\mathcal{H}$. Such transformations are called modular transformations. Their action on the torus is given by cutting the torus along a non-contractible loop, twisting and regluing. This corresponds to a large reparametrization which cannot be continously connected to the identity. Clearly, we have to require that string amplitudes are invariant under such large reparametrizations. This implies a consistency condition, known as modular invariance: the $\boldsymbol{\tau}$-integral in (86) must be invariant under modular transformations. This condition becomes non-trivial when considering more general background geometries or string theories with fermions.

The moduli space is obtained by restricting to a fundamental domain $\mathcal{F}$ of the action of $S l(2, \mathbf{Z})$ on $\mathcal{H}$. By modular invariance we can consistently restrict the $\tau$-integration to such an $\mathcal{F}$. The standard choice is found by looking at the action of the two generators of $\operatorname{Sl}(2, \mathbf{Z})$,

$$
\tau \rightarrow \tau+1, \quad \tau \rightarrow-\frac{1}{\tau} .
$$

Therefore the most convenient choice is

$$
\mathcal{F}=\left\{\tau \in \mathcal{H}\left|-\frac{1}{2} \leq \operatorname{Im}(\tau)<\frac{1}{2} \tau, \quad\right| \tau \mid \geq 1\right\}
$$

(with certain identifications along the boundary).

Modular invariance has deep consequences for the short distance behaviour of string theory. In fact, modular invariance is what makes closed string theories UV finite. To illustrate how this works, note that a one-loop amplitude in closed string theory takes the form

$$
A_{1 \text {-loop }}^{\text {String }} \sim \int_{\mathcal{F}} \frac{d^{2} \tau}{(\operatorname{Im}(\tau))^{2}} F(\tau) .
$$

An analogous expression for one loop amplitudes in quantum field theory is given by Schwinger's proper time parametrization,

$$
A_{1 \text {-loop }}^{\mathrm{QFT}} \sim \int_{\varepsilon}^{\infty} \frac{d t}{t} f(t),
$$

where $t$ is the proper time and $\varepsilon$ is an UV cutoff. In this formulation UV divergencies occure at short times $t \rightarrow 0$. In string theory $\operatorname{Im}(\tau)$ plays the role of proper time, and potential UV divergencies occure for $\operatorname{Im}(\tau) \rightarrow 0$. However, by restricting to the fundamental domain we have cut out the whole dangerous region of small times and high momenta. This confirms the intuitive idea that strings should have a particularly soft UV behaviour, because the theory has a minimal length scale, which works like a physical UV cutoff. Note that one still has IR divergencies. In bosonic string theory one has divergencies related to the tachyon, which show that the theory is unstable in Minkowski space. This problem is absent in supersymmetric string theories. In addition one can have IR divergencies related to massless states. Since there is only a finite number of massless string states, this problem has the same character as in field theory. 
Also note that the modular transformation $\tau \rightarrow-1 / \tau$ maps the UV region of $\mathcal{H}$ to its IR region. Thus, modular transformations map UV divergencies to IR divergencies and enable us to reinterpret them in terms of low energy physics (namely, intermediate massless states which go on-shell).

For higher genus surfaces $\Sigma_{g}$ with $g>1$ the story is similar, but more complicated. There is an analogue of the upper half plane, which is called Siegels upper half plane and has complex dimension $\frac{g(g+1)}{2}$. Since there are only $3 g-3$ complex moduli, this space contains more parameters then needed for $g \geq 4$. The Teichmüller space is embedded in a complicated way into Siegel's upper half plane. On top of this there is a modular group which has to be divided out.

Global conformal transformations. The integration over complex structure moduli in (36) reflects that surfaces with $g>0$ have metrics that cannot be related by reparametrizations. Therefore there is a finite left-over integration when replacing the integral over metrics by an integral over reparametrizations. For $g<2$ one has in addition the reciprocal phenomenon: these surfaces have global conformal isometries. This means that there are reparametrizations which do not change the metric, implying an overcounting of equivalent contributions in (86). Formally this is taken care of by the normalization factors $N_{0}^{\prime}, N_{1}^{\prime}$. The overcounting yields a multiplicative factor, which is the volume of the group of conformal isometries. This has to be cancelled by the normalization factors. For $g=0$ the conformal group is $S l(2, \mathbf{C})$ and has infinite volume. Thus one has to formally divide out an infinite constant. For $g=1$ the conformal group is $U(1)^{2}$, and has a volume which depends on the complex structure modulus $\tau$ of the world-sheet. This factor is crucial for world-sheet modular invariance.

The systematic approach is to treat the global conformal isometries as a residual gauge invariance and to apply the Faddeev-Popov technique.Then the volumes of residual gauge groups are properly taken care of. So far we have been sloppy about how and when to carry out the integration over the positions of the vertex operators. The proper order is as follows: one first carries out the $X^{\mu}$ integration to obtain a correlation function on a world-sheet of given topology and complex structure:

$$
\left\langle V_{1}\left(z_{1}, \bar{z}_{1}\right) \cdots\right\rangle_{g, \tau}=N_{g}^{\prime} \int D X e^{-S_{P}[X, \hat{h}(\boldsymbol{\tau})]} J(\hat{h}(\boldsymbol{\tau})) V_{1}\left(z_{1}, \bar{z}_{1}\right) \cdots
$$

Next one integrates over the positions of the vertex operators. For $g<2$ one treats the global conformal isometries by the Faddeev Popov method. The result is

$$
\left\langle V_{1} \cdots\right\rangle_{g, \boldsymbol{\tau}}=\int d \mu\left(z_{1}, \bar{z}_{1}, \ldots\right)\left\langle V_{1}\left(z_{1}, \bar{z}_{1}\right) \cdots\right\rangle_{g, \boldsymbol{\tau}},
$$

where $d \mu\left(z_{1}, \bar{z}_{1}, \ldots\right)$ for $g<2$ is a measure invariant under the global isometries.

For $g=0$ the measure vanishes if less than three vertex operators are present. This reflects the infinite volume of the global conformal group: by $S l(2, \mathbf{C})$ transformations one can map three points on the sphere to three arbitrary prescribed 
points. Thus, the $S l(2, \mathbf{C})$ symmetry can be used to by keep three vertex operators at fixed positions. In other words the first three integrations over vertex operators compensate the infinite volume of the global conformal group that one has to divide out. For less then three vertex operators one cannot compensate this infinite normalization factor and the result is zero. Thus, the integrated zero-, one- and two-point functions vanish. This implies that at string tree level the cosmological constant and all tadpoles diagrams vanish.

The final step in evaluating (86) is to integrate over complex structures and to sum over topologies:

$$
A(1, \ldots, M)=\sum_{g=0}^{\infty} \kappa^{M-\chi(g)} \int_{\mathcal{M}_{g}} d \mu(\boldsymbol{\tau})\left\langle V_{1} \ldots\right\rangle_{g, \boldsymbol{\tau}} .
$$

Through the vertex operators, $A(1, \ldots, M)$ is a function of the momenta $k_{i}^{\mu}$ and polarization tensors $\zeta_{i}^{\mu_{1} \cdots}$ of the external states.

Graviton scattering. Though we cannot go through the details of a calculation here, we would like to discuss the properties of string scattering amplitudes in a particular example. Our main interest being gravity, we choose the scattering of two massless closed string states. The corresponding external states are

$$
\zeta_{\mu \nu}^{(i)} \alpha_{-1}^{\mu} \tilde{\alpha}_{-1}^{\nu}\left|k^{(i)}\right\rangle
$$

with $i=1,2,3,4$. The resulting amplitude takes the following form:

$$
A_{4}^{\text {String }}=\kappa^{2} \frac{\Gamma\left(-\frac{\alpha^{\prime}}{4} s\right) \Gamma\left(-\frac{\alpha^{\prime}}{4} t\right) \Gamma\left(-\frac{\alpha^{\prime}}{4} u\right)}{\Gamma\left(1+\frac{\alpha^{\prime}}{4} s\right) \Gamma\left(1+\frac{\alpha^{\prime}}{4} t\right) \Gamma\left(1+\frac{\alpha^{\prime}}{4} u\right)} \cdot K\left(\zeta^{(i)}, k^{(i)}\right) .
$$

Here $s, t, u$ are the Mandelstam variables

$$
s=-\left(k^{(1)}+k^{(2)}\right)^{2}, \quad t=-\left(k^{(2)}+k^{(3)}\right)^{2}, \quad u=-\left(k^{(1)}+k^{(3)}\right)^{2}
$$

and $K\left(\zeta^{(i)}, k^{(i)}\right)$ is the kinematic factor, a complicated function of momenta and polarizations that we do not display.

Scattering amplitudes have poles whenever an intermediate states can be produced as a real physical state. Unitarity requires that the residue of the pole describing such a resonance is the product of the amplitudes of the subprocesses through which the intermediate state is produced and decays. In this way the pole structure of amplitudes is related to the particle spectrum of the theory.

The amplitude (98) has poles when the argument of one of the $\Gamma$-functions in the numerator takes a non-positive integer value,

$$
-\frac{\alpha^{\prime}}{4} x=0,-1,-2, \ldots, \quad \text { where } x=s, t, u .
$$

Comparing to the mass formula of closed strings,

$$
\alpha^{\prime} M^{2}=2(N+\tilde{N}-2)
$$


we see that the poles precisely correspond to massless and massive string states with $N=\tilde{N}=1,2,3, \ldots$. There is no pole corresponding to the tachyon $(N=$ 0 ) in this amplitude, because the tachyon cannot be produced as a resonance for kinematic reasons. When computing the amplitude for tachyon scattering instead, one also finds a tachyon pole.

The particular pole structure of (98) and of related string amplitudes was observed before the interpretation of the amplitudes in terms of strings was known. In the late 1960s it was observed experimentally that hadronic resonances obey a linear relation between the spin and the square of the mass, called Regge behaviour. This behaviour was correctly captured by the Veneziano amplitude, which has a structure similar to (98) and describes the scattering of two open string tachyons. The Regge behaviour was the clue for the interpretation of the Veneziano amplitudes and its cousins in terms of strings.

To see that string states show Regge behaviour, consider the truncation of string theory to four space-time dimension (which is consistent at tree level). Closed string states with level $N=\tilde{N}$ have spins $J \leq 2 N$, because the spin $J$ representation of the four-dimensional Lorentz group is the traceless symmetric tensor of rank $J$. Open string states have spins $J \leq N$. The states lie on lines in the $\left(M^{2}, J\right)$-plane, which are called Regge trajectories. The closed string Regge trajectories are given by

$$
\alpha_{\text {closed }}\left(M^{2}\right)=\alpha_{\text {closed }}^{\prime} M^{2}+\alpha_{\text {closed }}(0),
$$

where

$$
\alpha_{\text {closed }}^{\prime}=\frac{\alpha^{\prime}}{4}, \quad \alpha_{\text {closed }}(0)=1,0,-1, \ldots .
$$

String states correspond to those points on the Regge trajectories where $\alpha_{\text {closed }}^{\prime}\left(M^{2}\right)=N+\tilde{N}$. States with the maximal possible spin $J=2 N=N+$ $\tilde{N}$ for a given mass lie on the leading Regge trajectory $\alpha_{\text {closed }}(0)=1$. Since $\alpha^{\prime}$ determines the slope of the trajectories, it is called the Regge slope. The corresponding expressions for open strings are:

$$
\alpha_{\text {open }}\left(M^{2}\right)=\alpha_{\text {open }}^{\prime} M^{2}+\alpha_{\text {open }}(0)
$$

where

$$
\alpha_{\text {open }}^{\prime}=\alpha^{\prime}, \quad \alpha_{\text {open }}(0)=1,0,-1, \ldots .
$$

The resonances found in open string scattering lie on the corresponding Regge trajectories.

When computing scattering amplitudes in terms of Feynman diagrams in field theory, individual diagrams only have poles in one particular kinematic channel, i.e., in the s-channel or t-channel or u-channel. The full scattering amplitude, which has poles in all channels, is obtained by summing up all Feynman diagrams. In (closed oriented) string theory there is only one diagram in each order of perturbation theory, which simultanously has poles in all channels. The total amplitude can be written as a sum over resonances in one particular channel, say the s-channel. This is consistent with the existence of poles in the other 
channels, because there is an infinite set of resonances. When instead writing the amplitude in the form (98), it is manifestly symmetric under permutations of the kinematic variables $s, t, u$. This special property was called 'duality' in the old days of string theory (a term that nowadays is used for a variety of other, unrelated phenomena as well).

Another important property of (98) and other string amplitudes is that they fall off exponentially for large $s$, which means that the behaviour for large external momenta is much softer then in any field theory. This is again due to the presence of an infinite tower of excitations. Since loop amplitudes can be constructed by sewing tree amplitudes, this implies that the UV behaviour of loop diagrams is much softer than in field theory. This lead to the expectation that string loop amplitudes are UV finite, which was confirmed in the subsequent development of string perturbation theory.

Though we did not explicitly display the kinematic factor $K\left(\zeta^{(i)}, k^{(i)}\right)$ we need to emphasize one of its properties: it vanishes whenever one of the external states is a null state. As we learned above, null states have polarizations of the form

$$
\zeta_{\mu \nu}^{(i)}=k_{\mu}^{(i)} \xi_{\nu}^{(i)}+k_{\nu}^{(i)} \zeta_{\mu}^{(i)}
$$

and are gauge degrees of freedom. They have to decouple from physical scattering amplitudes, as it happens in the above example. This property is called 'on shell gauge invariance', because it is the manifestation of local gauge invariance at the level of scattering amplitudes. It can be proven to hold for general scattering amplitudes.

If we take the polarization tensors of the external states to be symmetric and traceless, then (98) describes graviton-graviton scattering. So far our identification of this string state with the graviton was based on its kinematic properties. Since Einstein gravity is the only known consistent interaction for a second rank, traceless symmetric tensor field ('massless spin-2-field'), we expect that this also holds dynamically. We will now check this explicitly. In the field theoretical perturbative approach to quantum gravity one starts from the Einstein-Hilbert action,

$$
S=\frac{1}{2 \kappa^{2}} \int d^{D} x \sqrt{g} R
$$

and expands the metric around flat space

$$
g_{\mu \nu}(x)=\eta_{\mu \nu}+\kappa \psi_{\mu \nu}(x) .
$$

The field $\psi_{\mu \nu}(x)$ is the graviton field. Expanding (107) in $\kappa$ one gets a complicated non-polynomial action for $\psi$ that one quantizes perturbatively. The resulting theory is non-renormalizable, but tree diagrams can be consistently defined and computed. In particular one can compute graviton-graviton scattering at tree level and compare it to the string amplitude (98). Denoting the field theory amplitude by $A_{4}^{\mathrm{FTh}}$, the relation is

$$
A_{4}^{\text {String }}=\frac{\Gamma\left(1-\frac{\alpha^{\prime}}{4} s\right) \Gamma\left(1-\frac{\alpha^{\prime}}{4} t\right) \Gamma\left(1-\frac{\alpha^{\prime}}{4} u\right)}{\Gamma\left(1+\frac{\alpha^{\prime}}{4} s\right) \Gamma\left(1+\frac{\alpha^{\prime}}{4} t\right) \Gamma\left(1+\frac{\alpha^{\prime}}{4} u\right)} A_{4}^{\mathrm{FTh}} .
$$


In the limit $\alpha^{\prime} \rightarrow 0$, which corresponds to sending the string mass scale to infinity, the string amplitude reduces to the field theory amplitude:

$$
\lim _{\alpha^{\prime} \rightarrow 0} A_{4}^{\text {String }}=A_{4}^{\mathrm{FTh}} .
$$

At finite $\alpha^{\prime}$ string theory deviates from field theory. The correction factor in (109) contains precisely all the poles corresponding to massive string states, whereas the massless poles are captured by the field theory amplitude. One can construct an effective action which reproduces the string amplitude order by order in $\alpha^{\prime}$. At order $\alpha^{\prime}$ one obtains four-derivative terms, in particular terms quadratic in the curvature tensor,

$$
S_{\mathrm{eff}}=\frac{1}{2 \kappa^{2}} \int d^{D} x \sqrt{g}\left(R+\alpha^{\prime} c_{1} R_{\mu \nu \rho \sigma} R^{\mu \nu \rho \sigma}+\cdots+\mathcal{O}\left(\left(\alpha^{\prime}\right)^{2}\right)\right),
$$

where $c_{1}$ is a numerical constant. The $\alpha^{\prime}$-expansion of the effective action is an expansion in derivatives. It is valid at low energies, i.e., at energies lower than the scale set by $\alpha^{\prime}$, where corrections due to massive string scales are small.

Obviously, it is very cumbersome to construct the effective action by matching field theory amplitudes with string amplitudes. In practice one uses symmetries to constrain the form of the effective action. This is particularly efficient for supersymmetric actions, which only depend on a few independent parameters or functions, which can be extracted from a small number of string amplitudes. A different technique, which often is even more efficient, is to study strings in curved backgrounds, and, more generally, in background fields.

\subsection{Strings in curved backgrounds}

So far we only discussed strings in flat backgrounds. Let us now consider the case of a curved background with Riemannian metric $G_{\mu \nu}(X)$. Then the Polyakov action takes the form of a non-linear sigma-model

$$
S_{P}=\frac{1}{4 \pi \alpha^{\prime}} \int d^{2} \sigma \sqrt{h} h^{\alpha \beta} \partial_{\alpha} X^{\mu} \partial_{\beta} X^{\nu} G_{\mu \nu}(X) .
$$

As emphasized above, the local Weyl invariance

$$
h_{\alpha \beta} \rightarrow e^{\Lambda(\sigma)} h_{\alpha \beta}
$$

is crucial for the consistency of string theory, since the construction of states, vertex operators and amplitudes is based on having a conformal field theory on the world-sheet. If the space-time metric is curved, then the Weyl invariance of the classical action (112) is still manifest. But at the quantum level it becomes non-trivial and imposes restrictions on $G_{\mu \nu}(X)$. In the non-linear sigma-model defined by (112) one can define a modified beta function $\bar{\beta}$, which measures the violation of local Weyl invariance. In order to have local Weyl invariance this function must vanish,

$$
\bar{\beta}=0 .
$$


Since $G_{\mu \nu}(X)$ are the field-dependent couplings of the non-linear sigma-model, the beta function $\bar{\beta}$ is a functional of $G_{\mu \nu}(X)$. It can be computed perturbatively, order by order in $\alpha^{\prime}$. The dimensionless expansion parameter is the curvature scale of the target space (i.e., space-time) measured in units of the string length $\sqrt{\alpha^{\prime}}$.

The leading term in this expansion is:

$$
\bar{\beta}_{\mu \nu}^{G}=-\frac{1}{2 \pi} R_{\mu \nu} .
$$

Thus the space-time background has to be Ricci-flat, i.e., it satisfies the vacuum Einstein equation. The condition imposed on the background field by local Weyl invariance on the world-sheet is its space-time equation of motion. This relation between world-sheet and space-time properties holds for other background fields as well and can be used as an efficient method to construct effective actions. One can also compute the $\alpha^{\prime}$-corrections to (115):

$$
\bar{\beta}_{\mu \nu}^{G}=-\frac{1}{2 \pi}\left(R_{\mu \nu}+\frac{\alpha^{\prime}}{2} R_{\mu \alpha \beta \gamma} R_{\nu}{ }^{\alpha \beta \gamma}\right) .
$$

The corresponding $\alpha^{\prime}$-corrections to the Einstein-Hilbert action take the form (111).

At this point we need to reflect a little bit on how gravity is described in string theory. So far we have seen that it enters in two ways: first, there is a graviton state $\zeta_{(\mu \nu)} \alpha_{-1}^{\mu} \tilde{\alpha}_{-1}^{\nu}|k\rangle$ in the string spectrum. Second, there is a background metric $G_{\mu \nu}(X)$. If gravity is described consistently, then these two objects must be related. To explore this we expand $G_{\mu \nu}(X)$ around flat space,

$$
G_{\mu \nu}(X)=\eta_{\mu \nu}+\kappa \psi_{\mu \nu}(X),
$$

and observe that the action (112) is related to the Polyakov action in flat space by

$$
S_{P}\left[G_{\mu \nu}\right]=S_{P}\left[\eta_{\mu \nu}\right]+\kappa V\left[\psi_{\mu \nu}\right],
$$

where

$$
V\left[\psi_{\mu \nu}\right]=\frac{1}{4 \pi \alpha^{\prime}} \int d^{2} \sigma \sqrt{h} h^{\alpha \beta} \psi_{\mu \nu}(X) \partial_{\alpha} X^{\mu} \partial_{\beta} X^{\nu} .
$$

Taking the Fourier transform of $\psi_{\mu \nu}(X)$ we obtain

$$
V\left[\psi_{\mu \nu}\right]=\frac{1}{4 \pi \alpha^{\prime}} \int d^{D} k \int d^{2} \sigma \sqrt{h} V_{\psi}(k, \tilde{\psi}(k))
$$

where

$$
V_{\psi}(k, \tilde{\psi}(k))=\tilde{\psi}_{\mu \nu}(k) \partial_{\alpha} X^{\mu} \partial^{\alpha} X^{\nu} e^{\mathrm{i} k_{\rho} X^{\rho}}
$$

is the graviton vertex operator with polarisation tensor $\tilde{\psi}_{\mu \nu}(k)$.

Thus the curved space action $S_{P}\left[G_{\mu \nu}\right]$ is obtained by deforming the flat space action $S_{P}\left[\eta_{\mu \nu}\right]$ by the graviton vertex operator. Since both actions must be conformal, $V[\psi]$ must be a so-called exactly marginal operator of the world-sheet 
field theory. These are the operators which generate deformations of the action while preserving conformal invariance. A necessary condition is that $V[\psi]$ must be a marginal operator, which means it has weights $(0,0)$ with respect to the original action. Such operators have the correct weight for being added to the action and generate infinitesimal deformations which preserve conformal invariance. Note that it is not guaranteed that a marginal operator is still marginal in the infinitesimally deformed theory. Only those marginal operators which stay marginal under deformation generate finite deformations of a conformal field theory and are called exactly marginal (or truly marginal).

If the integrated vertex operator $V[\psi]$ has weights $(0,0)$, then the vertex operator (121) must have weights $(1,1)$. This is the condition for a vertex operator to create a physical state. The resulting conditions on momenta and polarization are

$$
k^{2}=0, \quad k_{\mu} \tilde{\psi}^{(\mu \nu)}=0,
$$

which we now recognize as the Fourier transforms of the linearized Einstein equation. This the free part of the equations of motion for the graviton and characterizes its mass and spin.

Marginal operators are not necessarily exactly marginal. The flat space action defines a free field theory on the world-sheet, which is conformally invariant at the quantum level. Thus $V[\psi]$ is exactly marginal if and only if the curved space action $S_{P}\left[G_{\mu \nu}\right]$ is conformally invariant. By the beta-function analysis, this is equivalent to the full vacuum Einstein equation for the metric $G_{\mu \nu}$, plus corrections in $\alpha^{\prime}$. This is the full, non-linear equation of motion for the graviton string state.

In order to understand the relation between the graviton string state and the background metric even better we use (118) to relate amplitudes computed using the curved space action $S_{P}\left[G_{\mu \nu}\right]$ and the flat space action $S_{P}\left[\eta_{\mu \nu}\right]$ :

$$
\left\langle V_{1} \cdots V_{M}\right\rangle_{G}=\left\langle V_{1} \cdots V_{M} e^{V[\psi]}\right\rangle_{\eta}
$$

The operator $e^{V[\psi]}$ generates a coherent state of gravitons in flat space. This can be seen as follows: in quantum mechanics (think of the harmonic oscillator) coherent states are defined as states with minimal Heisenberg uncertainty. They are eigenstates of annihilation operators and can be constructed by exponentiating creation operators. The resulting states are not eigenstates of the number operator but are superpositions of states with all possible occupation numbers. In (123) the role of the creation operator is played by the graviton vertex operator.

In quantum field theory, coherent states are the states corresponding to classical fields. For example, in quantum electrodynamics a classical electrodynamic field can be represented as a coherent state of photons. Similarly, in gravity a curved metric (modulo global properties) can be described as a coherent state of gravitons in the Minkowski vacuum. This is realized in the above formula, where the amplitudes in the curved background can be computed equivalently by inserting the vertex operator for a coherent state of gravitons into the flat space amplitude. This is a manifestation of background independence: though 
we need to pick a particular background to define our theory, other backgrounds are different states in the same theory. Since consistent backgrounds must satisfy the equations of motion, one also calls them solutions of string theory. In this terminology different background geometries are different solutions of the single underlying string theory.

\subsection{Effective actions}

In the last section we have seen that the equation of motion of the metric/graviton can be obtained from an effective action. Such effective actions are very convenient, because they allow us to describe string states in terms of D-dimensional field theory. Effective actions are obtained in an expansion in $\alpha^{\prime}$ and therefore their use is limited to scales below the string scale. But given that the string scale probably is very large, they are extremely useful to extract particle physics or gravitational physics from string theory. Therefore they play a mayor role in string theory. We have also seen that there are two methods for deriving effective actions: the matching of string theory amplitudes with field theory amplitudes and solving the conditions for Weyl invariance $\bar{\beta}=0$ in a non-trivial background.

So far we found that the Einstein-Hilbert action is the leading part of the effective action for the graviton. We have seen that the closed string has two further massless modes, the dilaton $\Phi$ and the antisymmetric tensor field $B_{\mu \nu}$. We can now switch on the corresponding non-trivial background fields. The total world-sheet action is:

$$
S=S_{P}[G]+S[B]+S[\Phi] .
$$

Here $S_{P}[G]$ is the action (112),

$$
S[B]=\frac{1}{4 \pi \alpha^{\prime}} \int d^{2} \sigma \varepsilon^{\alpha \beta} \partial_{\alpha} X^{\mu} \partial_{\beta} X^{\nu} B_{\mu \nu}(X)
$$

and

$$
S[\Phi]=\frac{1}{4 \pi} \int d^{2} \sigma \sqrt{h} R^{(2)}(h) \Phi(X) .
$$

Here $\varepsilon^{\alpha \beta}$ is the totally antisymmetric world-sheet tensor density and $R^{(2)}(h)$ is the Ricci scalar of the world-sheet metric. Note that the dilaton action is higher order in $\alpha^{\prime}$.

The beta-function for the dilaton starts with a term proportional to $(D-26)$ and has $\alpha^{\prime}$-correction proportional to derivatives of $\Phi$. The leading term of the beta-function corresponds to a cosmological constant in the effective action. When considering string theory around backgrounds with constant dilaton, the only solution to the dilaton beta-function equation is to work in the critical dimension $D=26$. We will only consider such backgrounds here, and therefore the cosmological term in the effective action vanishes. But let us note that there are known exact solutions to the beta-function equations with non-constant dilaton. These describe exact string backgrounds with $D \neq 26$. 
Let us now return to dilaton term of the world-sheet action. When evaluated for constant dilaton, (126) is proportional to the Euler number of the worldsheet. For a Euclidean closed string world-sheet of genus $g$ we have:

$$
\chi=\frac{1}{4 \pi} \int_{\Sigma_{g}} d^{2} z \sqrt{h} R^{(2)}(h)=2-2 g .
$$

Therefore shifting the dilaton by a constant $a$,

$$
\Phi(X) \rightarrow \Phi(X)+a
$$

has the effect of shifting the total action (124) by a constant proportional to the Euler number:

$$
S \rightarrow S+a \chi(g) .
$$

For the corresponding partition function this is equivalent to rescaling the coupling by $e^{a}$ :

$$
Z=\sum_{g=0}^{\infty} \kappa^{-\chi(g)} \int D X D h e^{-S} \longrightarrow \sum_{g=0}^{\infty}\left(\kappa e^{a}\right)^{-\chi(g)} \int D X D h e^{-S}
$$

This shows that the coupling constant $\kappa$ and vacuum expectation value $\langle\Phi\rangle$ of the dilaton are not independent. To clarify the physical meaning of both quantities, we now investigate the effective action of the massless modes. The conditions for Weyl invariance of (124) are the Euler-Lagrange equation of the following effective action:

$$
S_{\mathrm{tree}}^{\mathrm{StrFr}}=\frac{1}{2 \kappa^{2}} \int d^{D} x \sqrt{G} e^{-2 \Phi}\left(R(G)-\frac{1}{12} H_{\mu \nu \rho} H^{\mu \nu \rho}+4 \partial_{\mu} \Phi \partial^{\mu} \Phi+\mathcal{O}\left(\alpha^{\prime}\right)\right) .
$$

This way of parametrizing the effective action is called the string-frame. The string frame metric $G_{\mu \nu}$ is the metric appearing in the world-sheet action (112). The field strength of the antisymmetric tensor field is

$$
H_{\mu \nu \rho}=3 ! \partial_{[\mu} B_{\nu \rho]},
$$

where $[\mu \nu \rho]$ denotes antisymmetrization.

Concerning the dilaton we note that its vacuum expectation value is not fixed by the equations of motion. Like in the partition function (130), shifting the dilaton by a constant is equivalent to rescaling the coupling. In order to determine the relation of the string coupling constant $\kappa$ to the physical gravitational coupling $\kappa_{\text {phys }}$ one has to perform a field redefinition that transforms the gravitational term in (131) into the standard Einstein-Hilbert action. The coefficient in front of this term is the physical gravitational coupling.

The transformation which achieves this is the following Weyl rescaling of the metric:

$$
g_{\mu \nu}:=G_{\mu \nu} e^{-\frac{4}{D-2}(\Phi-\langle\Phi\rangle)} .
$$


Expressing everything in terms of the Einstein frame metric $g_{\mu \nu}$ one obtains:

$S_{\text {tree }}^{\text {EinstFr }}=\frac{1}{2 \kappa_{\text {phys }}^{2}} \int \sqrt{g}\left(R(g)-\frac{1}{12} e^{-8 \frac{\Phi-\langle\Phi\rangle}{D-2}} H_{\mu \nu \rho} H^{\mu \nu \rho}-\frac{4}{D-2} \partial_{\mu} \Phi \partial^{\mu} \Phi+\mathcal{O}\left(\alpha^{\prime}\right)\right)$.

The physical gravitational coupling is

$$
\kappa_{\text {phys }}=\kappa e^{\langle\Phi\rangle} .
$$

Since the coupling $\kappa$ can be rescaled by shifting $\Phi$, it can be set to an arbitrary value. This is used to fix $\kappa$ :

$$
\kappa \stackrel{!}{=}\left(\alpha^{\prime}\right)^{\frac{D-2}{4}} .
$$

(Note that the $D$-dimensional gravitational couplings $\kappa, \kappa_{\text {phys }}$ have dimension (length) ${ }^{D-2 / 2}$.) Since $\kappa_{\text {phys }}$ and $\alpha^{\prime}$ are related by the vacuum expectation value of the dilaton we see that there is only one fundamental dimensionful parameter in string theory, which we can take to be either the gravitational coupling $\kappa_{\text {phys }}$ or the string scale set by $\alpha^{\prime}$. They are related by the vacuum expectation value of the dilaton, which classically is a free parameter labeling different ground states in one theory. Defining the dimensionless string coupling constant by

$$
g_{S}=e^{\langle\Phi\rangle},
$$

we have the relation

$$
\kappa_{\text {phys }}=\left(\alpha^{\prime}\right)^{\frac{D-2}{4}} g_{S} .
$$

The effective actions (131.134) have been constructed to leading order in $\alpha^{\prime}$ and at tree level in the string coupling $g_{S}$. Loop corrections in $g_{S}$ can be obtained, either by considering loop amplitudes or from the contribution of higher genus world-sheets to the Weyl anomaly (Fischler-Susskind mechanism). One might expect that loop corrections generate a potential for the dilaton and lift the vacuum degeneracy. But for the bosonic string one does not know the stable ground state, because of the tachyon. In supersymmetric string theories tachyons are absent, but no dilaton potential is created at any loop level. Thus the value of the string coupling remains a free parameter. This is (part of) the problem of vacuum degeneracy of superstring theories. Since the flatness of the dilaton potential is a consequence of supersymmetry, the solution of the vacuum degeneracy problem is related to understanding supersymmetry breaking.

For practical applications, both the string frame effective action and the Einstein frame effective action (and their higher-loop generalizations) are needed. The string frame action is adapted to string perturbation theory and has a universal dependence on the dilaton and on the string coupling:

$$
S_{\text {g-loop }}^{\text {StrFr }} \sim g_{S}^{-2+2 g} .
$$

The Einstein frame action is needed when analyzing gravitational physics, in particular for solutions of the effective action that describe black holes and other space-time geometries. Note that concepts such as the ADM mass of an 
asymptotically flat space-time are tied to the gravitational action written in the Einstein frame. The relation between the Einstein frame metric and the string frame metric is non-trivial, because it involves the dilaton, which in general is a space-time dependent field. Therefore various quantities, most importantly the metric itself, can take a very different form in the two frames. For example one metric might be singular wheras the other is not. In order to decide whether a field configuration is singular or not, one has of course to look at all the fields, not just at the metric. If the metric is singular in one frame but not in the other, then the dilaton must be singular.

\subsection{Interacting open and non-oriented strings}

We now indicate how the above results extend to open and non-oriented strings.

Open strings. The world-sheets describing the interactions of open strings have two kinds of boundaries: those corresponding to the initial and final strings and those corresponding to the motions of string endpoints. Boundaries corresponding to external strings can be mapped to punctures and are then replaced by vertex operators. The boundaries corresponding to the motions of string endpoints remain. They are the new feature compared to closed strings. Perturbation theory for open strings can then be developed along the same lines as for closed strings. Instead of closed oriented surfaces it involves oriented surfaces with boundaries, and the vertex operators for open string states are inserted at on the boundaries.

Again there is one fundamental interaction, which couples three open strings, and we assign to it a coupling constant $\kappa_{o}$. The most simple world-sheet, analogous to the sphere for closed strings, is the disc. It is leading in the expansion in $\kappa_{o}$ and describes scattering at tree level. The computation of tree level scattering amplitudes confirms the interpretation of the massless state as a gauge boson. The resulting effective action, to leading order in $\alpha^{\prime}$, is the Maxwell or, with Chan-Paton factors, the Yang-Mills action. It receives higher order corrections in $\alpha^{\prime}$ and one can show that the resulting actions are of Born-Infeld type.

Higher order diagrams in open string perturbation theory correspond to surfaces with more than one boundary component. They are obtained from the disc by removing discs from the interior. Each removal of a disc corresponds to an open string loop. The one loop diagram is the the annulus.

One can also introduce a coupling of two open strings to one closed string with coupling $\kappa_{o c}$ and consider theories of open and closed strings. Unitarity then implies that the three couplings $\kappa_{o}, \kappa_{o c}, \kappa$ are not independent. To see this consider first a disc diagram with two open string vertex operators at the boundaries and two closed string vertex operators in the interior. This amplitude can be factorized with an intermediate closed string. Looking at string interactions we see that one has one interaction between three closed strings and one between one closed and two open strings. Therefore the amplitude is proportional to $\kappa \kappa_{o c}$. The amplitude can also be factorized with an intermediate open string. 
This time one sees two interactions involving two open and one closed string. Therefore the amplitude is proportional to $\kappa_{o c}^{2}$. Comparing both forms of the amplitude we deduce

$$
\kappa \simeq \kappa_{O c}
$$

(the numerical factor has to be determined by explicit computation).

Next consider the open string one loop diagram, the annulus. Putting two vertex operators on each boundary one can again factorize it with either a closed or an open intermediate state. This way one finds

$$
\kappa \simeq \kappa_{o}^{2}
$$

Note that the above amplitude does not involve external closed string states. This indicates an important property of open string theories: the coupling to closed strings is not optional, but mandatory. When computing open string loop diagrams, one finds that they have poles which correspond to closed string states. Therefore consistency of open string theories at the quantum level requires the inclusion of closed strings. This means in particular that every consistent quantum string theory has to include gravity. The relation between open and closed strings becomes obvious when one realizes that the annulus is topologically equivalent to the cylinder. While the annulus intuitively is the open string one loop diagram, the cylinder is the closed string propagator. This is reflected by the properties of the corresponding string amplitudes, which can be written either as a sum over poles corresponding to open strings (open string channel) or as a sum over poles corresponding to closed strings (closed string channel).

The UV finiteness of closed string theories is due to modular invariance. Open string world-sheets do not have a modular group. The role of modular invariance is played by another property, called tadpole cancellation. The underlying observation is that the cancellation of divergencies between different diagrams is equivalent to the vanishing of the dilaton tadpole. It turns out that tadpole cancellation cannot be realized in a theory of oriented open and closed strings. In theories of non-oriented open and closed strings tadpole cancellation fixes the gauge group to be $S O\left(2^{D / 2}\right)$. For bosonic strings the critical dimension is $D=26$ an the gauge group must be $S O(8192)$. Since the primary problem of bosonic strings is the tachyon, it is not clear whether tadpole cancellation plays a fundamental role there. But for type I superstrings this is the condition which makes the theory finite.

Since we only discussed orientable world-sheets so far, we next collect some properties of the world-sheets of non-oriented strings.

Non-oriented strings. Theories of non-oriented strings are obtained by keeping only states which are invariant under world-sheet parity. Since such theories are insensitive to the orientation of the world-sheet one now has to include non-orientable world-sheets. Theories of closed non-oriented strings involve orientable and non-orientable surfaces without boundaries, whereas theories of open and closed non-oriented strings involve in addition orientable and non-orientable 
world-sheets with boundaries. Let us summarize which types of world-sheets occure in string theory, depending on boundary conditions and orientability of the world-sheet:

\begin{tabular}{|c|c|c|c|c|c|c|c|}
\hline \multicolumn{4}{|c|}{ Strings } & \multicolumn{4}{|c|}{ Surfaces } \\
\hline open & closed & oriented & non-oriented & $\begin{array}{c}\text { bounda } \\
\text { without }\end{array}$ & $\begin{array}{l}\text { aries } \\
\text { with }\end{array}$ & $\begin{array}{l}\text { orie } \\
\text { yes }\end{array}$ & $\begin{array}{l}\text { table } \\
\text { no }\end{array}$ \\
\hline $\mathrm{x}$ & - & $\mathrm{x}$ & - & $\mathrm{x}$ & - & $\mathrm{x}$ & - \\
\hline $\mathrm{x}$ & $\mathrm{x}$ & $\mathrm{x}$ & - & $\mathrm{x}$ & $\mathrm{x}$ & $\mathrm{x}$ & - \\
\hline $\mathrm{x}$ & - & - & $\mathrm{x}$ & $\mathrm{x}$ & - & $\mathrm{x}$ & $\mathrm{x}$ \\
\hline $\mathrm{x}$ & $\mathrm{x}$ & - & $\mathrm{x}$ & $\mathrm{x}$ & $\mathrm{x}$ & $\mathrm{x}$ & $\mathrm{x}$ \\
\hline
\end{tabular}

The simplest example of a non-orientable surface without boundary is the real projective plane $\mathbf{R} \mathbf{P}^{2}$, which is obtained from $\mathbf{R}^{2}$ by adding a circle at infinity, such that every line through the origin in $\mathbf{R}^{2}$ intersects the circle in one point. Equivalently, $\mathbf{R} \mathbf{P}^{2}$ is obtained from the disc by identifying antipodal points on its boundary. Thus, $\mathbf{R P}^{2}$ is a closed, but non-orientable surface, and it is a world-sheet occuring in theories of closed non-oriented strings. It is useful to note that $\mathbf{R P}^{2}$ can be obtained from the sphere, which is the tree-level worldsheet already familiar from oriented closed strings, by the following procedure: start with the sphere, remove a disc, (realize that the result is a disc itself,) then identify antipodal points on the resulting boundary. This operation is called 'adding a crosscap'. By iterating this process we get an infinite series of new non-orientable surfaces. For example, by adding a second crosscap we get the Klein bottle. As we discussed above, there is a similar operation that generates all orientable closed surfaces from the sphere: adding a handle. By adding both handles and crosscaps we can generate all closed surfaces, orientable and nonorientable. In fact, it is sufficient to either add handles (generating all orientable surfaces) or to add crosscaps (generating all non-orientable surfaces). The reason is that adding a crosscap and a handle is equivalent to adding three crosscaps.

When considering theories of non-oriented open strings one has to add worldsheets with boundaries. These are obtained from the world-sheets of closed strings by removing discs. For example, removing one disc from $\mathbf{R P}^{2}$ gives the Möbius strip. As we discussed in the last section, the couplings between open strings, $\kappa_{o}$, and between open and closed strings, $\kappa_{o g}$, are related to the closed string coupling $\kappa$ by unitarity. The order of a given world-sheet in string perturbation theory is $\kappa^{-\chi(g, b, c)}$, where the Euler number is now determined by the number $g$ of handles, the number $b$ of boundary components and the number $c$ of crosscaps:

$$
\chi(g, b, c)=2-2 g-b-c .
$$


Let us write down explicitly the first few world-sheets:

\begin{tabular}{|l|l|l|l||l||l|}
\hline$g$ & $b$ & $c$ & $\chi$ & Surface & Coupling \\
\hline \hline 0 & 0 & 0 & 2 & Sphere & $\kappa^{-2}$ \\
\hline 0 & 1 & 0 & 1 & Disc & $\kappa^{-1}$ \\
\hline 0 & 0 & 1 & 1 & Real projective plane & $\kappa^{-1}$ \\
\hline 1 & 0 & 0 & 0 & Torus & $\kappa^{0}$ \\
\hline 0 & 0 & 2 & 0 & Klein bottle & $\kappa^{0}$ \\
\hline 0 & 2 & 0 & 0 & Annulus = cylinder & $\kappa^{0}$ \\
\hline 0 & 1 & 1 & 0 & Möbius strip & $\kappa^{0}$ \\
\hline
\end{tabular}

\subsection{Further reading}

Vertex operators and the Polyakov path integral are discussed in all the standard textbooks [1,2,3, 3]. A very nice introduction to the use of conformal field theory in string theory is provided by [6]. A more detailed introduction to the Polyakov path integral can be found in $\mid \mathbb{8}$. For an extensive review of this subject, see [7]. A pedagogical treatment of the mathematical ingredients needed to treat higher genus surfaces can be found in [10], whereas [9] discusses the Polyakov path integral from the mathematicians point of view.

\section{Supersymmetric strings}

The bosonic string does not have fermionic states and therefore it cannot be used as a unified theory of particle physics and gravity. One way to introduce fermionic states is an extension known as the Ramond-Neveu-Schwarz string (RNS string). In this model one introduces new dynamical fields $\psi^{\mu}=\left(\psi_{A}^{\mu}\right)$ on the worldsheets, which are vectors with respect to space-time but spinors with respect to the world-sheet. We will suppress the world-sheet spinor indices $A=1,2$ most of the time. Surprisingly, the presence of such fields, when combined with a certain choice of boundary conditions, yields states which are spinors with respect to space-time, as we will see below.

The RNS model contains space-time bosons and fermions, but still has a tachyonic ground state. One then observes that there are projections of the spectrum which simultanously remove the tachyon and make the theories spacetime supersymmetric. A closer inspection shows that these projections are not optional, but required by consistency at the quantum level. This way one obtains three consistent supersymmetric strings theories, called type I, type IIA and type IIB. Finally there are also two so-called heterotic string theories, which are the result of a hybrid construction, combining type II and bosonic strings. This makes a total of five supersymmetric string theories. 


\subsection{The RNS model}

We now discuss the classical and quantum properties of the RNS string, proceeding along the same lines as we did for the bosonic string.

The RNS action. The action of the RNS model is obtained from the Polyakov action by extending it to an action with supersymmetry on the world-sheet. Note that that world-sheet supersymmetry is different from, and does not imply, supersymmetry in space-time. The action of the RNS model is constructed by extending the Polyakov action (4i) to an action with local world-sheet supersymmetry. This action also has local Weyl symmetry, and further local fermionic symmetries which make it locally superconformal. We will not need its explicit form here. The analogue of the conformal gauge is called superconformal gauge. In this gauge the action reduces to

$$
S_{\mathrm{RNS}}=\frac{1}{4 \pi \alpha^{\prime}} \int_{\Sigma} d^{2} \sigma\left(\partial_{\alpha} X^{\mu} \partial^{\alpha} X_{\mu}+\mathrm{i} \bar{\psi}^{\mu} \rho^{\alpha} \partial_{\alpha} \psi_{\mu}\right) .
$$

The fields $\psi^{\mu}=\left(\psi_{A}^{\mu}\right)$ are Majorana spinors with respect to the world-sheet and vectors with respect to space-time, while $\rho^{\alpha}=\left(\rho_{A B}^{\alpha}\right)$ are the two-dimensional spin matrices. We will usually suppress the world-sheet spinor index $A, B=1,2$. The action (145) is invariant under global world-sheet supersymmetry transformations:

$$
\delta X^{\mu}=\bar{\varepsilon} \psi^{\mu}, \quad \delta \psi^{\mu}=-\mathrm{i} \rho^{\alpha} \varepsilon \partial_{\alpha} X^{\mu} .
$$

The equations of motion are:

$$
\partial^{2} X^{\mu}=0, \quad \rho^{\alpha} \partial_{\alpha} \psi^{\mu}=0 .
$$

To these one has to add the constraints, which arise from the locally superconformal action. In this action the supersymmetric partner of the world-sheet metric is a vector-spinor, the gravitino. This field is non-dynamical in two dimensions and is set to zero in the superconformal gauge. The equation of motion for the metric implies that the energy-momentum tensor vanishes on shell:

$$
T_{\alpha \beta}=\partial_{\alpha} X^{\mu} \partial_{\beta} X_{\mu}+\frac{i}{2} \bar{\psi}^{\mu} \rho_{(\alpha} \partial_{\beta)} \psi_{\mu}-\text { Trace }=0 .
$$

The equation of motion for the gravitino implies that the world-sheet supercurrent $J_{\alpha}$ vanishes on shell:

$$
J_{\alpha}=\frac{1}{2} \rho^{\beta} \rho_{\alpha} \psi^{\mu} \partial_{\beta} X_{\mu}=0 .
$$

In order to solve the equation of motion for $\psi^{\mu}$ it is convenient to choose the following spin matrices:

$$
\rho^{0}=\left(\begin{array}{ll}
0 & \mathrm{i} \\
\mathrm{i} & 0
\end{array}\right), \quad \rho^{1}=\left(\begin{array}{ll}
0 & -\mathrm{i} \\
\mathrm{i} & 0
\end{array}\right) .
$$


Using the chirality matrix $\bar{\rho}=\rho^{0} \rho^{1}$ we see that the components $\psi_{ \pm}^{\mu}$ of $\psi^{\mu}$, defined by

$$
\psi^{\mu}=\left(\begin{array}{c}
\psi_{-} \\
\psi_{+}
\end{array}\right)
$$

with respect to the basis (150) are Majorana-Weyl spinors. The equations of motion decouple,

$$
\partial_{-} \psi_{+}^{\mu}=0, \quad \partial_{+} \psi_{-}^{\mu}=0,
$$

and have the general solution

$$
\psi_{+}^{\mu}=\psi_{+}^{\mu}\left(\sigma^{+}\right), \quad \psi_{-}^{\mu}=\psi_{-}^{\mu}\left(\sigma^{-}\right) .
$$

Next we have to specify the boundary conditions. Requiring the vanishing of the boundary terms when varying the action implies:

$$
\left.\left(\psi_{-}^{\mu} \delta \psi_{\mu-}-\psi_{+}^{\mu} \delta \psi_{\mu+}\right)\right|_{\sigma^{1}=0}=\left.\left(\psi_{-}^{\mu} \delta \psi_{-}^{\mu}-\psi_{+}^{\mu} \delta \psi_{\mu+}\right)\right|_{\sigma^{1}=\pi} .
$$

For open strings we take

$$
\begin{aligned}
& \psi_{+}^{\mu}\left(\sigma^{0}, \sigma^{1}=0\right)=\psi_{-}^{\mu}\left(\sigma^{0}, \sigma^{1}=0\right) \\
& \psi_{+}^{\mu}\left(\sigma^{0}, \sigma^{1}=\pi\right)= \pm \psi_{-}^{\mu}\left(\sigma^{0}, \sigma^{1}=\pi\right) .
\end{aligned}
$$

This couples $\psi_{+}^{\mu}$ and $\psi_{-}^{\mu}$ at the boundaries. Depending on the choice of sign in (156) one gets Ramond boundary conditions ('+' sign) or Neveu-Schwarz boundary conditions ('-' sign). One can use the same doubling trick that we used to obtain the Fourier expansion for bosonic open strings. Setting

$$
\psi^{\mu}\left(\sigma^{0}, \sigma^{1}\right):=\left\{\begin{array}{l}
\psi_{-}^{\mu}\left(\sigma^{0},-\sigma^{1}\right) \text { if }-\pi \leq \sigma^{1} \leq 0, \\
\psi_{+}^{\mu}\left(\sigma^{0}, \sigma^{1}\right) \text { if } 0 \leq \sigma^{1} \leq \pi,
\end{array}\right.
$$

we find that $\psi$ is periodic for $\mathrm{R}$ (amond)-boundary conditions and antiperiodic for N(eveu-)S(chwarz)-boundary conditions on the doubled world-sheet. Consistency at the loop level requires that both types of boundary conditions have to be included. The Hilbert space has both an NS-sector and an R-sector.

For closed strings we can make $\psi_{+}$and $\psi_{-}$either periodic (R-boundary conditions) or antiperiodic (NS-boundary conditions):

$$
\begin{aligned}
& \psi_{+}^{\mu}\left(\sigma^{0}, \sigma^{1}=\pi\right)= \pm \psi_{+}^{\mu}\left(\sigma^{0}, \sigma^{1}=0\right), \\
& \psi_{-}^{\mu}\left(\sigma^{0}, \sigma^{1}=\pi\right)= \pm \psi_{-}^{\mu}\left(\sigma^{0}, \sigma^{1}=0\right) .
\end{aligned}
$$

Since $\psi_{+}^{\mu}$ and $\psi_{-}^{\mu}$ are independent, one has four different choices of fermionic boundary conditions: R-R, NS-R, R-NS, NS-NS. Again considerations at the loop level require that all four sectors have to be included.

We can now write down solutions of (152) subject to the boundary conditions that we admit. For open strings we use the doubling trick and Fourier expand (157). For R-boundary conditions one obtains,

$$
\psi_{\mp}^{\mu}=\frac{1}{\sqrt{2}} \sum_{n \in \mathbf{Z}} d_{n}^{\mu} e^{-\mathrm{i} n \sigma^{\mp}},
$$


while for NS-boundary conditions the result is:

$$
\psi_{\mp}^{\mu}=\frac{1}{\sqrt{2}} \sum_{r \in \mathbf{Z}+\frac{1}{2}} b_{r}^{\mu} e^{-\mathrm{i} r \sigma^{\mp}} .
$$

For closed strings R-boundary conditions in the right-moving sector we get:

$$
\psi_{-}^{\mu}=\sum_{n \in \mathbf{Z}} d_{n}^{\mu} e^{-2 \mathrm{i} n \sigma^{-}}
$$

while with NS-boundary conditions this becomes

$$
\psi_{-}^{\mu}=\sum_{r \in \mathbf{Z}+\frac{1}{2}} b_{r}^{\mu} e^{-2 \mathrm{i} r \sigma^{-}} .
$$

The Fourier coefficients of the left-moving fields are denoted $\tilde{d}_{n}^{\mu}$ and $\tilde{b}_{r}^{\mu}$, respectively.

Likewise, one obtains Fourier coefficients of the energy momentum tensor $T_{\alpha \beta}$ and of the supercurrent $J_{\alpha}$. For open strings the Fourier coefficients of $J_{+}, J_{-}$(in the doubled intervall) are denoted $F_{m}$ in the R-sector and $G_{r}$ in the NS-sector. For closed strings the Fourier modes of $J_{+}$are denoted $F_{m}, G_{r}$, while those of $J_{-}$are $\tilde{F}_{m}$ and $\tilde{G}_{r}$. The Fourier components of $T_{++}$and $T_{--}$are denotes as before.

Covariant quantization of the RNS model. The covariant quantization of the RNS model proceeds along the lines of the bosonic string. We will consider open strings for definiteness. The canonical commutation relations of the $\alpha_{m}^{\mu}$ are as before. The fermionic modes satisfy the canonical anticommutation relations

$$
\left\{b_{r}^{\mu}, b_{s}^{\nu}\right\}=\eta^{\mu \nu} \delta_{r+s, 0}
$$

in the NS-sector and

$$
\left\{d_{m}^{\mu}, d_{n}^{\nu}\right\}=\eta^{\mu \nu} \delta_{m+n, 0}
$$

in the R-sector. (For closed strings there are analogous relations for the second set of of modes.)

The Virasoro generators get contributions from both the bosonic and the fermionic oscillators, $L_{m}=L_{m}^{(\alpha)}+L_{m}^{(N S) /(R)}$. The bosonic part $L_{m}^{(\alpha)}$ is given by (29), while the contributions from the fermionic oscillators in the respective sectors are:

$$
\begin{aligned}
& L_{m}^{(N S)}=\frac{1}{2} \sum_{r=-\infty}^{\infty}\left(r+\frac{1}{2} m\right) b_{r} \cdot b_{m+r} \\
& L_{m}^{(R)}=\frac{1}{2} \sum_{n=-\infty}^{\infty}\left(n+\frac{1}{2} m\right) d_{n} \cdot d_{m+n}
\end{aligned}
$$


The explicit formulae for the modes of the supercurrent are:

$$
\begin{aligned}
G_{r} & =\sum_{n=-\infty}^{\infty} \alpha_{-n} \cdot b_{r+n}, \\
F_{m} & =\sum_{n=-\infty}^{\infty} \alpha_{-n} \cdot d_{m+n} .
\end{aligned}
$$

The modes of $T_{\alpha \beta}$ and $J_{\alpha}$ generate a supersymmetric extension of the Virasoro algebra. In the NS sector this algebra takes the form

$$
\begin{aligned}
& {\left[L_{m}, L_{n}\right]=(m-n) L_{m+n}+\frac{D}{8}\left(m^{3}-m\right) \delta_{m+n, 0},} \\
& {\left[L_{m}, G_{r}\right]=\left(\frac{1}{2} m-r\right) G_{m+r},} \\
& \left\{G_{r}, G_{s}\right\}=2 L_{r+s}+\frac{D}{2}\left(r^{2}-\frac{1}{4}\right) \delta_{r+s, 0},
\end{aligned}
$$

while in the R-sector one finds

$$
\begin{aligned}
& {\left[L_{m}, L_{n}\right]=(m-n) L_{m+n}+\frac{D}{8} m^{3} \delta_{m+n, 0},} \\
& {\left[L_{m}, F_{n}\right]=\left(\frac{1}{2} m-n\right) F_{m+n},} \\
& \left\{F_{m}, F_{n}\right\}=2 L_{m+n}+\frac{D}{2} m^{2} \delta_{m+n, 0} .
\end{aligned}
$$

The subspace of physical states $\mathcal{F}_{\text {phys }} \subset \mathcal{F}$ is found by imposing the corresponding super Virasoro constraints. In the NS-sector the constraints are:

$$
\begin{array}{ll}
L_{n}|\Phi\rangle & =0, \quad n>0, \\
\left(L_{0}-a\right)|\Phi\rangle & =0, \\
G_{r}|\Phi\rangle & =0, \quad r>0 .
\end{array}
$$

Absence of negative norm states is achieved for

$$
D=10 \text { and } a=\frac{1}{2} .
$$

(Like for bosonic strings there is the option to have a non-critical string theory with $D<10$, which we will not discuss here.) Thus the critical dimension has been reduced to 10 .

In the R-sector the constraints are:

$$
\begin{array}{ll}
L_{n}|\Phi\rangle & =0, \quad n>0, \\
\left(L_{0}-a\right)|\Phi\rangle & =0, \\
F_{n}|\Phi\rangle & =0, \quad n \geq 0 .
\end{array}
$$

Note that there is no normal ordering ambiguity in $F_{0}$. Since $F_{0}^{2}=L_{0}$ we conclude $a=0$. The critical dimension is 10 , as in the NS-sector:

$$
D=10 \text { and } a=0 \text {. }
$$


Let us construct explicitly the lowest states of the open string in both sectors. In the NS-sector the basic momentum eigenstates satisfy

$$
\begin{aligned}
& \alpha_{m}^{\mu}|k\rangle=0, \quad m>0, \\
& b_{r}^{\mu}|k\rangle=0, \quad r>0
\end{aligned}
$$

and the constraint $\left(L_{0}-\frac{1}{2}\right)|\Phi\rangle=0$ provides the mass formula:

$$
\alpha^{\prime} M^{2}=N-\frac{1}{2},
$$

where we reinstated $\alpha^{\prime}$. The number operator gets an additional term $N^{(b)}$ compared to 39, which counts fermionic oscillations:

$$
\begin{aligned}
N^{(d)} & =\sum_{r=1 / 2}^{\infty} r b_{-r} \cdot b_{r}, \\
{\left[N, b_{-r}^{\mu}\right] } & =r b_{-r}^{\mu} .
\end{aligned}
$$

Now we can list the states:

\begin{tabular}{|l|l|l|}
\hline Occupation & Mass & State \\
\hline \hline$N=0$ & $\alpha^{\prime} M^{2}=-\frac{1}{2}$ & $|k\rangle$ \\
\hline$N=\frac{1}{2}$ & $\alpha^{\prime} M^{2}=0$ & $b_{-1 / 2}^{\mu}|k\rangle$ \\
\hline$N=1$ & $\alpha^{\prime} M^{2}=\frac{1}{2}$ & $\begin{array}{l}b_{-1 / 2}^{\mu} b_{-1 / 2}^{\nu}|k\rangle \\
\alpha_{-1}^{\mu}|k\rangle\end{array}$ \\
\hline$N=\frac{3}{2}$ & $\alpha^{\prime} M^{2}=1$ & $\begin{array}{l}b_{-1 / 2}^{\mu} b_{-1 / 2}^{\nu} b_{-1 / 2}^{\rho}|k\rangle \\
\alpha_{-1}^{\mu} b_{-1 / 2}^{\nu}|k\rangle \\
b_{-3 / 2}^{\mu}|k\rangle\end{array}$ \\
\hline
\end{tabular}

Thus the NS-sector of the open string consists of space-time bosons and has a tachyonic ground state. The massless state is a gauge boson.

The basic momentum eigenstates in the R-sector defined by:

$$
\begin{array}{ll}
\alpha_{m}^{\mu}|k\rangle=0, & m>0, \\
d_{m}^{\mu}|k\rangle=0, & m>0 .
\end{array}
$$

The constraint $L_{0}|\Phi\rangle=0$ yields the mass formula

$$
\alpha^{\prime} M^{2}=N .
$$

The number operator gets an additional fermonic contribution

$$
N^{(d)}=\sum_{m=1}^{\infty} m d_{-m} \cdot d_{m} .
$$


The zero modes $d_{0}^{\mu}$ of the fermionic fields play a distinguished role. Their algebra is, up to normalization, the Clifford algebra $C l(1,9)$ :

$$
\left\{d_{0}^{\mu}, d_{0}^{\nu}\right\}=\eta^{\mu \nu} .
$$

The unique irreducible representation of this algebra is the spinor representation of the Lorentz group $S O(1,9)$. Introducing standard Clifford generators $\gamma^{\mu}=$ $\sqrt{2} d_{0}^{\mu}$, the generators of the spinor representation are $\sigma^{\mu \nu}=\frac{1}{4}\left[\gamma^{\mu}, \gamma^{\nu}\right]$. Since the $d_{0}^{\mu}$ are real, this representation is the 32 -dimensional Majorana representation, denoted [32].

The zero modes $d_{0}^{\mu}$ commute with the number operator. Therefore the states in the R-sector organize themselves into spinor representations of the Lorentz group. This is how space-time spinors are described in the RNS model. To construct the states, we denote the ground state of the R-sector by

$$
|a\rangle, \quad a=1, \ldots, 32=2^{D / 2},
$$

where $a$ transforms in the [32] representation. Then the first states are:

\begin{tabular}{|l|l|l|}
\hline Occupation & Mass & State \\
\hline \hline$N=0$ & $\alpha^{\prime} M^{2}=0$ & $|a\rangle$ \\
\hline$N=1$ & $\alpha^{\prime} M^{2}=1$ & $d_{-1}^{\mu}|a\rangle$ \\
& & $\alpha_{-1}^{\mu}|a\rangle$ \\
\hline
\end{tabular}

The constraints $L_{n}|\Phi\rangle=0(n>0)$ and the new constraints $F_{n}|\Phi\rangle=0(n \geq 0)$ impose restrictions on the polarization. For example, $F_{0}|a\rangle=0$ is easily seen to be the Fourier transform of the massless Dirac equation and reduces the number of independent components by a factor $\frac{1}{2}$. Excited states are obtained by acting with creation operators $\alpha_{-m}^{\mu}, d_{-m}^{\mu}$ on the gound state. Since the product of a tensor representation of the Lorentz group with a spinor representation always gives spinor representations, we see that all states in the R-sector are space-time spinors.

The GSO projection for open strings. The RNS model solves the problem of describing space-time fermions but still has a tachyon. Gliozzi, Scherk and Olive observed that one can make a projection of the spectrum, which removes the tachyon. Moreover the resulting spectrum is supersymmetric in the spacetime sense. This so-called GSO projection is optional at the classical level, but it becomes mandatory at the quantum level, as we will discuss below.

The GSO projector in the NS-sector is defined as follows:

$$
P_{G S O}^{(N S)}=-(-1)^{\sum_{r=1 / 2}^{\infty} b_{-r} \cdot b_{r}} .
$$

Imposing $P_{G S O}^{(N S)} \stackrel{!}{=} 1$, one projects out all the states which contain an even number of $b_{-r}^{\mu}$ creation operators. This in particular removes the tachyon. The 
GSO projector in the R-sector is

$$
P_{G S O}^{(R)}=\bar{\gamma}(-1)^{\sum_{m=1}^{\infty} d_{-m} \cdot d_{m}},
$$

where $\bar{\gamma}$ is the ten-dimensional chirality operator. On the ground state $|a\rangle$ of the R-sector the projection $P_{G S O}^{(R)}|\Phi\rangle \stackrel{!}{=} 1$ removes one chirality of the spinor. This is consistent, because in ten space-time dimensions the irreducible spinor representations are Majorana-Weyl spinors. The [32] representation decomposes according to

$$
[32]=[16]_{+}+[16]_{-} .
$$

With the GSO projection one only keeps one chirality (which we have taken to be the $[16]_{+}$, for definiteness):

$$
|a\rangle=\left|a_{+}\right\rangle+\left|a_{-}\right\rangle \longrightarrow\left|a_{+}\right\rangle,
$$

where $a_{+}=1, \ldots, 16$ is a Majorana-Weyl index.

At the massive level just projecting out one chirality would not be consistent, as massive particles cannot be chiral. The projection with (194) keeps states which either have ' + ' chirality and an even number of $d_{-m}^{\mu}$ creation operators or ' -' chirality and an odd number of $d_{-m}^{\mu}$ creation operators.

By writing down the first few states one can easily verify that after the projection the NS-sector and R-sector have an equal number of states, and that the massive states in the R-sector combine into full (non-chiral) massive Lorentz representations.

Checking the equality of states at every mass level is done by computing the one-loop partition function. Moreover one can construct explicitly the representation of the ten-dimensional super Poincaré algebra on the physical states. This is done using BRST techniques and lies beyond the scope of these lectures. Here we restrict ourselves to noting that the ground state of the open string, after GSO projection, is a ten-dimensional vector supermultiplet:

$$
\left\{b_{-1 / 2}^{\mu}|k\rangle, \quad\left|a_{+}\right\rangle\right\}
$$

Spectrum and GSO projection for closed strings. Let us next study the spectrum of closed RNS strings. The masses of states are determined by

$$
\begin{aligned}
& \alpha^{\prime} M^{2}=2\left(N-a_{x}+\tilde{N}-\tilde{a}_{x}\right), \\
& N-a_{x}=\tilde{N}-\tilde{a}_{x},
\end{aligned}
$$

with normal ordering constants $a_{R}=0=\tilde{a}_{R}$ and $a_{N S}=\frac{1}{2}=\tilde{a}_{N S}$. 
We start by listing the first states in the NS-NS sector:

\begin{tabular}{|l|l|l|}
\hline Occupation & Mass & States \\
\hline \hline$N=\tilde{N}=0$ & $\alpha^{\prime} M^{2}=-2$ & $|k\rangle$ \\
\hline$N=\tilde{N}=\frac{1}{2}$ & $\alpha^{\prime} M^{2}=0$ & $b_{-1 / 2}^{\mu} \tilde{b}_{-1 / 2}^{\nu}|k\rangle$ \\
\hline$N=\tilde{N}=1$ & $\alpha^{\prime} M^{2}=2$ & $\alpha_{-1}^{\mu} \tilde{\alpha}_{-1}^{\nu}|k\rangle$ \\
& & $\begin{array}{l}\alpha_{-1}^{\mu} \tilde{b}_{-1 / 2}^{\nu} \tilde{b}_{-1 / 2}^{\rho}|k\rangle \\
b_{-1 / 2}^{\mu} b_{-1 / 2}^{\nu} \tilde{\alpha}_{-1}^{\rho}|k\rangle \\
\end{array}$ \\
& & $b_{-1 / 2}^{\mu} b_{-1 / 2}^{\nu} \tilde{b}_{-1 / 2}^{\rho} \tilde{b}_{-1 / 2}^{\sigma}|k\rangle$ \\
\hline
\end{tabular}

All these states are bosons, and at the massless level we recognize the graviton, the dilaton and the antisymmetric tensor.

In the R-R sector, the ground state transforms in the [32] $\times[32]$ representation and is denoted $|a, \tilde{a}\rangle$. The first states are

\begin{tabular}{|l|l|l|}
\hline Occupation & Mass & State \\
\hline \hline$N=\tilde{N}=0$ & $\alpha^{\prime} M^{2}=0$ & $|a, \tilde{a}\rangle$ \\
\hline$N=\tilde{N}=1$ & $\alpha^{\prime} M^{2}=2$ & $\alpha_{-1}^{\mu} \tilde{\alpha}_{-1}^{\nu}|a, \tilde{a}\rangle$ \\
& & $d_{-1}^{\mu} \tilde{\alpha}_{-1}^{\nu}|a, \tilde{a}\rangle$ \\
& & $\alpha_{-1}^{\mu} \tilde{d}_{-1}^{\nu}|a, \tilde{a}\rangle$ \\
& & $d_{-1}^{\mu} \tilde{d}_{-1}^{\nu}|a, \tilde{a}\rangle$ \\
\hline
\end{tabular}

The product of two spinor representations is a vector-like representations. Therefore the states in the $\mathrm{R}-\mathrm{R}$ sector are bosons. In more detail, the $[32] \times[32] \mathrm{rep}-$ resentation is the direct sum of all the antisymmetric tensor representations of rank zero to ten. Using the ten-dimensional $\Gamma$-matrices we can decompose a general massless state into irreducible representations:

$$
\left|\Phi_{R R}\right\rangle=\left(F \delta_{a \tilde{a}}+F_{\mu} \Gamma_{a \tilde{a}}^{\mu}+F_{\mu \nu} \Gamma_{a \tilde{a}}^{\mu \nu}+\cdots\right)|a, \tilde{a}\rangle .
$$

By evaluating the remaining constraints $F_{0}\left|\Phi_{R R}\right\rangle=0=\tilde{F}_{0}\left|\Phi_{R R}\right\rangle$ one obtains the conditions

$$
k^{\mu_{1}} F_{\mu_{1} \mu_{2} \ldots \mu_{n}}=0 \text { and } k_{\left[\mu_{0}\right.} F_{\left.\mu_{1} \mu_{2} \ldots \mu_{n}\right]}=0,
$$

which are the Fourier transforms of the equation of motion and Bianchi identity of an $n$-form field strength:

$$
d \star F_{(n)}=0 \text { and } d F_{(n)}=0
$$

The physical fields are antisymmetric tensor gauge fields or rank $n-1$. Note that in contrast to the antisymmetric NS-NS field, the states in the R-R sector (and 
the corresponding vertex operators) describe the field strength and not the gauge potential. When analyzing interactions one finds that there are no minimal gauge couplings but only momentum couplings of these fields (i.e. couplings involving the field strength). In other words the perturbative spectrum does not contain states which are charged under these gauge fields. This is surprising, but a closer analysis shows that the theory has solitonic solutions which carry R-R charge. These so called R-R charged p-branes turn out to be an alternative description of D-branes.

Now we turn to the NS-R sector. The first states are:

\begin{tabular}{|l|l|l|}
\hline Occupation & Mass & State \\
\hline \hline$N=\frac{1}{2}, \tilde{N}=0$ & $\alpha^{\prime} M^{2}=0$ & $b_{-1 / 2}^{\mu}|\tilde{a}\rangle$ \\
\hline$N=\frac{3}{2}, \tilde{N}=1$ & $\alpha^{\prime} M^{2}=4$ & $\alpha_{-1}^{\mu} b_{-1 / 2}^{\nu} \tilde{\alpha}_{-1}^{\rho}|\tilde{a}\rangle$ \\
& & $b_{-1 / 2}^{\mu} b_{-1 / 2}^{\nu} b_{-1 / 2}^{\rho} \tilde{\alpha}_{-1}^{\sigma}|\tilde{a}\rangle$ \\
& & $\alpha_{-1}^{\mu} b_{-1 / 2}^{\nu} \tilde{d}_{-1}^{\rho}|\tilde{a}\rangle$ \\
& & $b_{-1 / 2}^{\mu} b_{-1 / 2}^{\nu} b_{-1 / 2}^{\rho} \tilde{d}_{-1}^{\mu}|\tilde{a}\rangle$ \\
\hline
\end{tabular}

The massless state is a product of a vector $[D]$ and a spinor $\left[2^{D / 2}\right]$. It decomposes into a vector-spinor and a spinor:

$$
[D] \times\left[2^{D / 2}\right]=\left[(D-1) 2^{D / 2}\right]+2^{D / 2} .
$$

Therefore this state and all other states in the NS-R sector are space-time fermions. The spectrum of the R-NS sector is obtained by exchanging left- and right-moving fermions.

We observe that the massless states contains two vector-spinors. The only known consistent interaction for such fields is supergravity. There these fields are called gravitini. They sit in the same supermultiplet as the graviton, they are the gauge fields of local supertransformations and couple to the conserved supercurrent. The spectrum of the closed RNS model is obviously not supersymmetric. This suggests that we have to make a projection in order to obtain consistent interactions. This brings us to the GSO projection for closed strings, which makes the spectrum supersymmetric and removes the tachyon. The GSO projection is applied both in the left-moving and in the right-moving sector. In the R-sectors one has to decide which chirality one keeps. There are two inequivalent projections of the total spectrum: one either takes opposite chiralities of the R-groundstates (type A) or the same chiralities (type B). The resulting theories are the type IIA and type IIB superstring. Let us look at their massless states. The NS-NS sectors of both theories are identical. The states

$$
b_{-1 / 2}^{\mu} \tilde{b}_{-1 / 2}^{\nu}|k\rangle
$$

are the graviton $G_{\mu \nu}$ the dilaton $\Phi$ and the antisymmetric tensor $B_{\mu \nu}$. The number of on-shell states is $8 \cdot 8=64$. The ground states of the R-R sectors are:

$$
\left|a_{+}, \tilde{a}_{-}\right\rangle \text {(type A), }
$$




$$
\left|a_{+}, \tilde{a}_{+}\right\rangle \text {(type B) . }
$$

In both cases we have $8 \cdot 8=64$ on-shell states. Again we can decompose these representations into irreducible antisymmetric tensors. For type IIA we get a two-form and a four-form field strength, corresponding to a one-form and a three-form potential:

$$
\text { IIA : } A_{\mu}, A_{\mu \nu \rho} \text {. }
$$

There is also a zero-form field strength which has no local dynamics. It corresponds to the so-called massive deformation of IIA supergravity, which is almost but not quite a cosmological constant. (In the effective action the corresponding term is a dimensionful constant multiplied by the dilaton. This is as close as one can get to a cosmological constant in ten-dimensional supergravity.)

In the IIB theory one has a one-form, a three-form and a selfdual five-form field strength. The corresponding potentials are:

$$
\text { IIB : } A, A_{\mu \nu}, A_{\mu \nu \rho \sigma} .
$$

The massless states in the NS-R sector and R-NS sector are:

$$
\begin{array}{lll}
\text { IIA }: & b_{-1 / 2}^{\mu}\left|\tilde{a}_{-}\right\rangle & \tilde{b}_{-1 / 2}^{\mu}\left|a_{+}\right\rangle, \\
\text {IIB : } b_{-1 / 2}^{\mu}\left|\tilde{a}_{+}\right\rangle & \tilde{b}_{-1 / 2}^{\mu}\left|a_{+}\right\rangle,
\end{array}
$$

The total number of fermionic states is 128 in both cases. The decomposition into irreducible representations gives two vector-spinors, the gravitini, and two spinors, called dilatini. For type IIA they have opposite chiralities, whereas for type IIB they have the same chiralities. The corresponding space-time fields are:

$$
\begin{array}{ll}
\text { IIA : } & \psi_{+}^{\mu}, \psi_{-}^{\mu}, \psi_{+}, \psi_{-}, \\
\text {IIB : } & \psi_{+(1)}^{\mu}, \psi_{+(2)}^{\mu}, \psi_{+(1)}, \psi_{+(2)} .
\end{array}
$$

All together we get the field content of the type IIA/B supergravity multiplet with 128 bosonic and 128 fermionic on shell states. The IIA theory is non-chiral whereas the IIB theory is chiral. The massive spectra are of course non-chiral, and, moreover, they are identical.

\subsection{Type I and type II superstrings}

We will now begin to list all consistent supersymmetric string theories. A priori, we have the following choices: strings can be (i) open or closed, (ii) oriented or non-oriented, (iii) one can make the GSO projection, with two inequivalent choices (type A and B) for closed strings and (iv) one can choose gauge groups for open strings: $U(n)$ for oriented and $S O(n)$ or $U s p(2 n)$ for non-oriented strings.

We have already seen that not all combinations of these choices are consistent at the quantum level. Since theories of open strings have closed string poles in loop diagrams, we can either have closed or closed and open strings. The next restriction comes from modular invariance. On the higher genus world-sheets 
of closed oriented strings, one has to specify boundary conditions around every handle. Since modular invariance maps one set of boundary conditions to others, these choices are not independent. It turns out that one has to include both NSand R-boundary conditions around every handle, but one has the freedom of choosing relative signs between different orbits of action of the modular group on the set of boundary conditions. There are four possible choices. Two of them correspond to the IIA and IIB superstrings. The other two choices are nonsupersymmetric theories without fermions, known as type $0 \mathrm{~A}$ and $\mathrm{OB}$, which we will not discuss here.

Type IIA and IIB are theories of oriented closed strings. Can we construct supersymmetric string theories with oriented closed and open strings? The states of the oriented open string fall into representations of the minimal $N=1$ supersymmetry algebra in $D=10$. This algebra has 16 supercharges, which transform as a Majorana-Weyl spinor under the Lorentz group. In ten dimensions there are two further supersymmetry algebras, called $N=2 A$ and $N=2 B$. They have 32 supercharges which either combine into two Majorana-Weyl spinors of opposite chirality (A) or into two Majorana-Weyl spinors of the same chirality (B). The states of the oriented closed string form multiplets of the $N=2 A$ or $N=2 B$ supersymmetry algebra. In particular one has two gravitini, which must couple to two independent supercurrents. Therefore oriented open and closed strings cannot be coupled in a supersymmetric way. One can also show that any such theory has divergencies, due to the presence of dilaton tadpoles.

Next we have to consider non-oriented strings. A theory of non-oriented closed strings can be obtained by projecting the type IIB theory onto states invariant under world-sheet parity. (IIA is not invariant under world-sheet parity, because the R-groundstates have opposite chirality.) This theory has divergencies, which are related to the non-vanishing of dilaton tadpole diagrams. One can also see from the space-time point of view that this theory is inconsistent: the massless states form the $N=1$ supergravity multiplet, which is chiral. Pure $N=1$ supergravity has a gravitational anomaly, which can only be cancelled by adding precisely 496 vector multiplets.

Therefore we have to look at theories with non-oriented closed and open strings. Tadpole cancellation precisely occurs if the gauge group is chosen to be $S O\left(2^{D / 2}\right)=S O(32)$. This is one of the gauge groups for which gravitational anomalies cancel. The other anomaly-free gauge groups are $E_{8} \times E_{8}, E_{8} \times U(1)^{248}$ and $U(1)^{496}$, which, however, cannot be realized through Chan-Paton factors. Thus there is one supersymmetric string theory with non-oriented closed and open strings and gauge group $S O(32)$. This is the type I superstring.

Let us construct the massless spectrum of this theory. The closed string sector is obtained by projecting the IIB theory onto states invariant under world-sheet parity. Parity acts by exchanging left- and right-moving quantities:

$$
\alpha_{m}^{\mu} \leftrightarrow \tilde{\alpha}_{m}^{\mu}, \quad b_{r}^{\mu} \leftrightarrow \tilde{b}_{r}^{\mu}, \quad d_{m}^{\mu} \leftrightarrow \tilde{d}_{m}^{\mu}, \quad\left|a_{+}\right\rangle \leftrightarrow\left|\tilde{a}_{+}\right\rangle .
$$

The action on the R-R ground state is:

$$
\left|a_{+}, \tilde{a}_{+}\right\rangle \leftrightarrow-\left|\tilde{a}_{+}, a_{+}\right\rangle .
$$


The '-' sign reflects that one exchanges two fermionic states. (To make this precise one needs to construct the so-called spin fields $S^{a}, S^{\tilde{a}}$ which generate the R-groundstates from the NS-groundstate. This can be done in the framework of BRST quantization, which we did not introduce here.)

We can now write down the massless states of the type IIB string which are invariant under world-sheet parity and survive the projection. In the NS-NS sector we find

$$
\text { NS-NS : } \quad \frac{1}{2}\left(b_{-1 / 2}^{\mu} \tilde{b}_{-1 / 2}^{\nu}+b_{-1 / 2}^{\nu} \tilde{b}_{-1 / 2}^{\mu}\right)|k\rangle .
$$

Therefore the $B_{\mu \nu}$ field is projected out and we are left with the graviton $G_{\mu \nu}$ and dilaton $\Phi$. In the R-R sector the invariant massless state is:

$$
\text { R-R : } \quad \frac{1}{2}\left(\left|a_{+}, \tilde{a}_{+}\right\rangle-\left|\tilde{a}_{+}, a_{+}\right\rangle\right) .
$$

Thus only the antisymmetric part of the tensor product of the two MajoranaWeyl spinors survives the projection. This corresponds to the three-form field strength $F_{\mu \nu \rho}$, as is most easily seen by computing the dimensions of the representations. Thus the two-form R-R gauge field $A_{\mu \nu}$ survives the projection.

In the NS-R and R-NS one finds the following invariant state:

$$
\text { R-NS/NS-R : } \frac{1}{2}\left(b_{-1 / 2}^{\mu}\left|\tilde{a}_{+}\right\rangle+\tilde{b}_{-1 / 2}^{\mu}\left|a_{+}\right\rangle\right) .
$$

Therefore one gravitino $\psi_{+}^{\mu}$ and one dilatino $\psi^{\mu}$ are kept.

In the NS-sector of the open string we get massless vectors $A_{\mu}^{i}$, which transform in the adjoint representations of $S O(32): i=1, \ldots, \operatorname{dim}(\operatorname{adj} S O(32))=496$. The R-sector contains massless spinors $\psi^{i}$ which combine with the vectors to form vector supermultiplets.

Combining the massless states of the closed and open string sector we get the field content of $N=1$ supergravity coupled to Super-Yang-Mills theory with gauge group $S O(32)$.

\subsection{Heterotic strings}

There is yet another construction of supersymmetric string theories. It is a hybrid construction, which combines the bosonic string with the type II superstring and is called the heterotic string. The right-moving sector is taken from the type II superstring, whereas the left-moving sector is taken from the bosonic string. To get a modular invariant theory, the sixteen extra left-moving coordinates have to be identified periodically,

$$
X^{I} \simeq X^{I}+w_{(i)}^{I}, \quad I=1, \ldots, 16 .
$$

The vectors $\boldsymbol{w}_{(i)}=\left(w_{(i)}^{I}\right), i=1, \ldots, 16$ generate a sixteen dimensional lattice $\Gamma_{16}$. Modular invariance requires that $\Gamma_{16}$ is an even self-dual lattice. Modulo rotations, there are only two such lattices, the root lattice of $E_{8} \times E_{8}$ and the 
lattice generated by the roots and the weights of one of the Majorana-Weyl spinor representations of $S O(32)$. Thus, there are two different heterotic string theories.

The bosonic massless states come from the NS-sector and take the form

$$
\begin{aligned}
& \alpha_{-1}^{\mu} \tilde{b}_{-1 / 2}^{\nu}|k\rangle \\
& \alpha_{-1}^{I} \tilde{b}_{-1 / 2}^{\nu}|k\rangle \\
& e^{\mathrm{i} k_{I}^{(i)} x_{L}^{I} \tilde{b}_{-1 / 2}^{\nu}|k\rangle}
\end{aligned}
$$

Here $\alpha_{-1}^{I}$ are the oscillators corresponding to the sixteen extra left-moving directions. The vectors $k^{(i)}=\left(k_{I}^{(i)}\right)$ are discrete momentum vectors in the extra dimensions. The above states are massless if the vectors $k^{(i)}$ have norm-squared two. The two lattices $\Gamma_{16}$ have 480 such vectors, corresponding to the roots of $E_{8} \times E_{8}$ and $S O(32)$, respectively. Together with the states generated by the internal oscillators one gets bosons in the adjoint representations of theses two groups. The massless fermionic states are obtained by replacing $\tilde{b}_{-1 / 2}^{\nu}|k\rangle$ by the R-ground state $\left|a_{+}\right\rangle$. In total one gets the $N=1$ supergravity multiplet plus vector multiples in the adjoint representation of $E_{8} \times E_{8}$ or $S O(32)$.

The massless sectors of the five supersymmetric string theories correspond to four different supergravity theories. The type I and the heterotic string with gauge group $S O(32)$ have the same massless spectrum, but their massive spectra and interactions are different.

Let us summarize the essential properties of the five supersymmetric string theories:

\begin{tabular}{|c|c|c|c|c|c|}
\hline Type & open/closed? & oriented? & chiral? & supersymmetry & gauge group \\
\hline \hline I & both & no & yes & $N=1$ & $S O(32)$ \\
\hline II A & closed & yes & no & $N=2 A$ & - \\
\hline II B & closed & yes & yes & $N=2 B$ & - \\
\hline Heterotic & closed & yes & yes & $N=1$ & $E_{8} \times E_{8}$ \\
\hline Heterotic & closed & yes & yes & $N=1$ & $S O(32)$ \\
\hline
\end{tabular}

\subsection{Further reading}

Supersymmetric string theories are discussed in all of the standard textbooks [1, 2, 3. 1.5 . To prove the necessity of the GSO projection and the consistency of the heterotic string as a perturbative quantum theory one needs properties of the multiloop path integral [7]. A paedagogical treatment of the relation between the GSO projection and boundary conditions in the path integral can be found in [6]. 


\section{5 p-Branes in type II string theories}

In this section we discuss a class of solitons of the type II string theories, which turn out to be alternative descriptions of the D-branes introduced earlier.

\subsection{Effective actions of type II string theories}

The effective actions for the massless states of type IIA/B superstring theory are the corresponding type IIA/B supergravity actions. Since we will be interested in bosonic solutions of the field equations, we will only display the bosonic parts. The effective action for the fields in the NS-NS sector is the same for both theories. Moreover it is identical to the effective action of the bosonic string:

$$
S_{\mathrm{NS}-\mathrm{NS}}=\frac{1}{2 \kappa^{2}} \int d^{10} x \sqrt{-G} e^{-2 \Phi}\left(R+4 \partial_{\mu} \Phi \partial^{\mu} \Phi-\frac{1}{12} H_{\mu \nu \rho} H^{\mu \nu \rho}\right) .
$$

The R-R sectors consist of antisymmetric tensor gauge fields. For an $(n-1)$ form gauge potential $A_{(n-1)}$ with field strength $F_{(n)}=d A_{(n-1)}$ the generalized Maxwell action is

$$
S \simeq-\frac{1}{2} \int F_{(n)} \wedge \star F_{(n)}=-\frac{1}{2} \int d^{D} x \sqrt{-G}\left|F_{(n)}\right|^{2}
$$

where

$$
\left|F_{(n)}\right|^{2}:=\frac{1}{n !} F_{\mu_{1} \cdots \mu_{n}} F^{\mu_{1} \cdots \mu_{n}}
$$

In the effective R-R actions one has in addition Chern-Simons terms.

In the IIA theory the R-R fields are $A_{(1)}$ and $A_{(3)}$. It is convenient to define a modified field strength

$$
\tilde{F}_{(4)}=d A_{(3)}-A_{(1)} \wedge H_{(3)},
$$

where $H_{(3)}=d B_{(2)}$ is the field strength of the antisymmetric NS-NS tensor field. Then the R-R action is the sum of a Maxwell and a Chern-Simons term:

$$
\begin{aligned}
S_{\mathrm{R}-\mathrm{R}}^{\mathrm{IIA}}= & -\frac{1}{4 \kappa^{2}} \int d^{10} x \sqrt{-G}\left(\left|F_{(2)}\right|^{2}+\left|\tilde{F}_{(4)}\right|^{2}\right) \\
& -\frac{1}{4 \kappa^{2}} \int B_{(2)} \wedge F_{(4)} \wedge F_{(4)} .
\end{aligned}
$$

The massless R-R fields of IIB string theory are $A_{(0)}, A_{(2)}$ and $A_{(4)}$. Again it is useful to define modified field strengths

$$
\begin{aligned}
& \tilde{F}_{(3)}=F_{(3)}-A_{(0)} \wedge H_{(3)}, \\
& \tilde{F}_{(5)}=F_{(5)}-\frac{1}{2} A_{(2)} \wedge H_{(3)}+\frac{1}{2} B_{(2)} \wedge F_{(3)} .
\end{aligned}
$$

Since $\tilde{F}_{(5)}$ must be selfdual, the kinetic term (225) vanishes and does not give a field equation. The simplest way out is to impose the selfduality condition only 
at the level of the equation of motion. Then one can use the action

$$
\begin{aligned}
S_{\mathrm{R}-\mathrm{R}}^{\mathrm{IIB}}= & -\frac{1}{4 \kappa^{2}} \int d^{10} x \sqrt{-G}\left(\left|F_{(1)}\right|^{2}+\left|\tilde{F}_{(3)}\right|^{2}+\frac{1}{2}\left|\tilde{F}_{(5)}\right|^{2}\right) \\
& -\frac{1}{4 \kappa^{2}} \int A_{(4)} \wedge H_{(3)} \wedge F_{(3)} .
\end{aligned}
$$

The correct covariant equations of motion result when varying the action and imposing selfduality of $\tilde{F}_{(5)}$ afterwards.

\section{$5.2 \quad$ R-R charged p-brane solutions}

The type II effective actions have static solutions which are charged under the R$\mathrm{R}$ gauge fields. The solution charged under $A_{(p+1)}$ has $p$ translational isometries. From far it looks like a p-dimensional membrane and therefore one calls it a pbrane solution or just a p-brane.

For $0 \leq p \leq 2$ the solution has the following form:

$$
\begin{aligned}
d s_{\text {Str }}^{2}= & H^{-1 / 2}(r)\left(-d t^{2}+\left(d x^{1}\right)^{2}+\cdots+\left(d x^{p}\right)^{2}\right) \\
& +H^{1 / 2}(r)\left(\left(d x^{p+1}\right)^{2}+\cdots+\left(d x^{9}\right)^{2}\right) \\
F_{(p+2)}= & d H^{-1}(r) \wedge d t \wedge d x^{1} \wedge \cdots \wedge d x^{p} \\
e^{-2 \Phi}= & H^{(p-3) / 2}(r),
\end{aligned}
$$

where

$$
r^{2}=\left(x^{p+1}\right)^{2}+\cdots+\left(x^{9}\right)^{2}
$$

and $H(r)$ is a harmonic function of the transverse coordinates $\left(x^{p+1}, \ldots, x^{9}\right)$ :

$$
\Delta^{\perp} H=\sum_{i=p+1}^{9} \partial_{i} \partial_{i} H=0 .
$$

We require that the solution becomes asymptotically flat at transverse infinity and normalize the metric such that it approaches the standard Minkowski metric. This fixes

$$
H(r)=1+\frac{Q_{p}}{r^{7-p}} .
$$

$Q_{p}$ measures the flux of the R-R field strength at transverse infinity. A convenient way to parametrize it is:

$$
Q_{p}=N_{p} c_{p}, \quad c_{p}=\frac{(2 \pi)^{7-p}}{(7-p) \omega_{8-p}}\left(\alpha^{\prime}\right)^{\frac{7-p}{2}} g_{S} .
$$

$N_{p}$ is a constant, which a priori is real, but will turn out later to be an integer. Therefore $c_{p}$ is the fundamental quantum of R-R p-brane charge. $\omega_{n}$ is the volume of the n-dimensional unit sphere,

$$
\omega_{n}=\frac{2 \pi^{(n+1) / 2}}{\Gamma\left(\frac{n+1}{2}\right)} .
$$


Besides geometrical factors, $Q_{p}$ contains the appropriate power of $\alpha^{\prime}$ to give it the correct dimension. $g_{S}$ is the dimensionless string coupling. Note that in the above solution for the dilaton we have subtracted the dilaton vacuum expectation value from $\Phi$.

The metric used in this solution is the string frame metric, as indicated by the subscript. (The effective action was also given in the string frame.) Using (133) we can find the corresponding Einstein frame metric:

$$
\begin{aligned}
d s_{\text {Einst }}^{2}= & -H^{\frac{p-7}{8}}(r)\left(-d t^{2}+\left(d x^{1}\right)^{2}+\cdots+\left(d x^{p}\right)^{2}\right) \\
& +H^{\frac{p+1}{8}}(r)\left(\left(d x^{p+1}\right)^{2}+\cdots+\left(d x^{9}\right)^{2}\right) .
\end{aligned}
$$

The above solution is most easily understood as a generalization of the extreme Reissner-Nordstrom solution of four-dimensional Einstein-Maxwell theory. Let us review its properties.

The isometry directions $t, x^{1}, \ldots, x^{p}$ are called longitudinal or world-volume directions, the others transverse directions. Since the solution has translational invariance, it has infinite mass, as long as one does not compactify the worldvolume directions. However, the tension $T_{p}$ (the energy per world volume) is finite. Since the solution becomes asymptotically flat in the transverse directions, the tension can be defined by a generalization of the ADM construction of general relativity. Concretely, the tension of a p-brane can be extracted from the Einstein frame metric by looking at the leading deviation from flatness:

$$
g_{00}=-1+\frac{16 \pi G_{N}^{(D)} T_{p}}{(D-2) \omega_{D-2-p} r^{D-3-p}}+\cdots=-1+\frac{16 \pi G_{N}^{(10)} T_{p}}{8 \omega_{8-p} r^{7-p}}+\cdots
$$

The Schwarzschild radius $r_{S}$ of the brane is:

$$
r_{S}^{D-3-p}=\frac{16 \pi G_{N}^{(D)} T_{p}}{(D-2) \omega_{D-2-p}} .
$$

Since there is only one independent dimensionful constant, which we take to be $\alpha^{\prime}$, we can express the ten-dimensional Newton constant $G_{N}^{(10)}$ in terms of $\alpha^{\prime}$ and the dimensionless string coupling $g_{S}$ :

$$
G_{N}^{(10)}=8 \pi^{6}\left(\alpha^{\prime}\right)^{4} g_{S}^{2} .
$$

Since Newton's constant is related to the physical gravitational coupling by

$$
8 \pi G_{N}^{(D)}=\kappa_{(D), \text { phys }}^{2}
$$

in any dimension, this corresponds to replacing the conventional choices (136, 138) by $\kappa^{2} \stackrel{!}{=} 64 \pi^{7}\left(\alpha^{\prime}\right)^{4}$ and $\kappa_{\text {phys }}^{2}=64 \pi^{7}\left(\alpha^{\prime}\right)^{4} g_{S}^{2}$.

Using (238) we can compute the tension of the p-brane solution (231):

$$
T_{p}=\frac{N_{p}}{g_{S}\left(\alpha^{\prime}\right)^{\frac{p+1}{2}}(2 \pi)^{p}} .
$$


For $r \rightarrow 0$ the solution (231) has a null singularity, that is a curvature singularity which is lightlike and coincides with an event horizon. The p-brane (231) is the extremal limit of a more general black p-brane solution, which has a time-like singularity along a p-dimensional surface and a regular event horizon. In the extremal limit, the singularity and the even horizon coincide. This behaviour is similar to the Reissner-Nordstrom black hole. The behaviour of the black pbrane in the extremal limit is slightly more singular, because for the extremal Reissner-Nordstrom black hole singularity and horizon do not coinicide. But since the singularity of the p-brane solution is not naked, we can think about it as describing an extended charged black hole. The charge (density) carried under the gauge field $A_{(p+1)}$ can be read off from the asymptotic behaviour of the field strength,

$$
F_{01 \ldots p} \simeq \frac{Q_{p}}{r^{8-p}}
$$

Instead of $Q_{p}$ we can define use a redefined charge, which has the dimension of a tension:

$$
\hat{Q}_{p}=\frac{1}{2 \kappa^{2}} \oint_{S_{8-p}} \star F_{(p+2)},
$$

which gives

$$
\hat{Q}_{p}=N_{p} \frac{\mu_{p}}{g_{S}}, \quad \mu_{p}=\frac{1}{(2 \pi)^{p}\left(\alpha^{\prime}\right)^{\frac{p+1}{2}}} .
$$

We now observe that tension and charge are equal:

$$
T_{p}=\hat{Q}_{p}
$$

More generally, black p-brane solutions satisfy the Bogomol'nyi bound

$$
T_{p} \geq \hat{Q}_{p}
$$

This inequality guarantees the existence of an event horizon, just as for charged black holes.

A feature that distinguishes our solutions from Reissner-Nordstrom type black holes is that one also has a non-trivial scalar, the dilaton.

The extremal solution has a multicentered generalization. When replacing $H(r)$ by

$$
H\left(\boldsymbol{x}_{\perp}\right)=1+\sum_{i=1}^{N} \frac{\left|Q_{p}^{(i)}\right|}{\left|\boldsymbol{x}_{\perp}-\boldsymbol{x}_{\perp}^{(i)}\right|^{7-p}},
$$

one still has a static solution, provided that all the charges $Q_{p}^{(i)}$ have the same sign. Here $\boldsymbol{x}_{\perp}=\left(x^{p+1}, \ldots, x^{9}\right)$ and $\boldsymbol{x}_{\perp}^{(i)}$ is the position of (the horizon of) the i-th p-brane. It is remarkable that the solution is static for arbitrary positions $\boldsymbol{x}_{\perp}^{(i)}$, because this implies that the gravitational attraction and the 'electrostatic' repulsion cancel for arbitrary distances. (If one flips the sign of one charge, one has to flip the corresponding tension, which makes the solution unphysical.) Systems of extremal Reissner-Nordstrom black holes have the same properties. 
The corresponding multi-centered solutions are known as Majumdar-Papapetrou solutions.

The remarkable properties of these (and other related) solutions can be understood in terms of supersymmetry. The solution (231) is a supersymmetric solution, i.e., it has Killing spinors. Killing spinors are the supersymmetric analogues of Killing vectors $v(x)$, which satisfy

$$
\left.\mathcal{L}_{v(x)} \Psi(x)\right|_{\Psi_{0}(x)}=0,
$$

where $\mathcal{L}$ is the Lie derivative. Here $\Psi(x)$ collectively denotes all the fields, and $\Psi_{0}(x)$ is the particular field configuration, which is invariant under under the transformation generated by the vector field $v(x)$. In supergravity theories one can look for field configurations $\Psi_{0}(x)$ which are invariant under supersymmetry transformations. From the action one knows the supersymmetry variations of all the fields, $\delta_{\varepsilon(x)} \Psi(x)$, where the spinor (field) $\varepsilon(x)$ is the transformation parameter. Then one can plugg in a given field configuration $\Psi_{0}(x)$ and check whether the variation vanishes for a specific choice of $\varepsilon(x)$ :

$$
\left.\delta_{\varepsilon(x)} \Psi(x)\right|_{\Psi_{0}(x)}=0 .
$$

Since the supersymmetry transformations involve derivatives of $\varepsilon(x)$, this is a system of first order differential equation for $\varepsilon(x)$. Solutions of (250) are called Killing spinors.

The type II superalgebras have 32 independent real transformation parameters, which organize themselves into two Majorana-Weyl spinors $\varepsilon_{i}(x)$. The equation (250) fixes the space-time dependence of the $\varepsilon_{i}(x)$. For the p-brane one finds

$$
\varepsilon_{i}(x)=g_{t t}^{1 / 4}(x) \varepsilon_{i}^{(0)},
$$

where the constant Majorana-Weyl spinors $\varepsilon_{i}^{(0)}, i=1,2$ are related by

$$
\varepsilon_{2}^{(0)}=\Gamma^{0} \cdots \Gamma^{p} \varepsilon_{1}^{(0)} .
$$

Since half of the components of the $\varepsilon_{i}^{(0)}$ is fixed in terms of the other half, we see that we have 16 independent solutions, i.e., 16 Killing spinors. The maximal number of Killing spinors equals the number of sypersymmetry transformation parameters, which is 32 in type II theory. Solutions with the maximal number of Killing spinors are invariant under all supersymmetry transformations. They are the analogues of maximally symmetric spaces in Riemannian geometry, which by definition have as many isometries as flat space. One example of a maximally supersymmetric solution of type II theory is flat ten-dimensional Minkowski space. Here the Killing spinor equation is solved by all constant spinors. The p-brane solution (231) has 16 Killing spinors, and only is invariant under half of the supersymmetry transformations. Solutions with residual supersymmetry are called BPS solutions, and solutions which preserve half of the supersymmetry are called ' $\frac{1}{2}$ BPS solutions'. 
The Bogomol'nyi bound 247 can be shown to follow from supersymmetry. In this context it is then also called the BPS bound. In theories where the supersymmetry algebra contains central charges, 247) is a relation between the mass or tension of a state and its central charge. In our case the charges carried under the R-R gauge fields are such central charges. The representations of the supersymmetry algebra fall into distinct classes, depending on whether they saturate the bound or not. Representations which saturate the bound are called short representations or BPS representations. Since BPS states have the minimal tension possible for their charge they are absolutely stable. This minimization of energy also accounts for the existence of static multicentered solutions.

So far we restricted ourselves to p-branes solutions with $0 \leq p \leq 2$. There is a second class, where the solution (231) and the other formulae take the form form, but with $p$ replaced by $\tilde{p}$ with $4 \leq \tilde{p} \leq 6$. The field strength $F_{\tilde{p}+2}$ in equation (231) is the $\star$-dual of $F_{p+2}=d A_{p+1}$. Since $F_{\tilde{p}+2}=\star F_{p+2}$ implies $p+\tilde{p}+4=D=10$, each of the so-called electric solutions with $p=0,1,2$ has a dual magnetic solution with $\tilde{p}=6,5,4$.

There is also a solution with $p=3$. The five-form gauge field is selfdual, and the solution for $F_{5}$ is from (231) by adding the $\star$-dual of the right hand side of the equation. The solutions for the metric and for the dilaton are not modified. Note that for $p=3$ the dilaton is constant. The three-brane solution is not singular at $r=0$. Instead one has a regular horizon, and the geometry is asymptotic to $A d S^{5} \times S^{5}$. This geometry has 32 Killing spinors and is fully supersymmetric. The interior of this geometry is isometric to the exterior, in particular it is non-singular. Since the field strength is selfdual, the three-brane carries an equal amount of electric and magnetic charge (it is not only dyonic, carrying both electric and magnetic charge, but selfdual).

Electric and magnetic charges are subject to a generalized Dirac quantization condition, which can be found by generalizing either the Dirac string or the $\mathrm{Wu}$ Yang construction known from four-dimensional magnetic monopoles. In our conventions the condition is:

$$
(2 \pi)^{7} g_{S}^{2}\left(\alpha^{\prime}\right)^{4} \hat{Q}_{p} \hat{Q}_{\tilde{p}} \in 2 \pi \mathbf{Z}
$$

This fixes the possible magnetic charges in terms of the electric charges. Using T-duality and S-duality one can fix the electric and magnetic charge units. Tduality is a symmetry that can be proven to hold in string perturbation theory. It acts on our solutions by transforming p-branes into $(\mathrm{p} \pm 1)$-branes. In this way one can relate the tensions and charges of all $\mathrm{R}-\mathrm{R}$ charged p-branes. S-duality is a conjectured non-perturbative symmetry of IIB string theory. It relates the $\mathrm{R}-\mathrm{R}$ one-brane to a solution which describes the fundamental IIB string. This way one relates the fundamental unit of $\mathrm{R}-\mathrm{R}$ one-brane charge to the charge carried by a fundamental IIB string under the NS-NS B-field. The resulting R$\mathrm{R}$ p-brane charge units are given by $\mu_{p}$ (245) and satisfy Dirac quantization in a minimal way: $\mu_{p} \mu_{\tilde{p}}(2 \pi)^{7}\left(\alpha^{\prime}\right)^{4}=2 \pi$. Thus $N_{p}$ in (245) is an integer which counts multiples of the fundamental R-R charge. When using $Q_{p}$ instead of $\hat{Q}_{p}$ to measure charges then $c_{p}$ as defined in (235) is the unit charge. 
We now summarize the R-R charged p-brane solutions of type II string theories:

\begin{tabular}{|l|l|l|l|}
\hline Theory & R-R potential & electric sol. & magnetic sol. \\
\hline \hline IIA & $A_{(1)}$ & $p=0$ & $p=6$ \\
\hline IIB & $A_{(2)}$ & $p=1$ & $p=5$ \\
\hline IIA & $A_{(3)}$ & $p=2$ & $p=4$ \\
\hline IIB & $A_{(4)}$ & $p=3$ (selfdual) \\
\hline
\end{tabular}

The R-R p-brane solutions have properties which qualify them as solitons: They are static, stable (BPS bound), regular (no naked singularities) solutions of the field equations and have finite tension. The three-brane has an additional property familiar from two-dimensional solitons: it interpolates between two vacua, Minkowski space at infinity and $A d S^{5} \times S^{5}$ at the event horizon. (We call $A d S^{5} \times S^{5}$ a vacuum, because it is maximally supersymmetric.) For solitons one expects that the tension depends on the coupling as $T \sim \frac{1}{g^{2}}$. This is, for example, what one finds for monopoles in Yang-Mills-Higgs theories. In this respect the R-R p-branes show an unusal behaviour as their tension is proportional to the inverse coupling, $T_{p} \sim \frac{1}{g_{S}}$, see (242). This behaviour is in between the one expected for a soliton $T \sim \frac{1}{g_{S}^{2}}$ and the one of a fundamental string, $T \sim 1$, which is independent of the coupling.

One clue to this unexpected behaviour is that the fundamental coupling of three closed strings is - up to a constant - the square of the coupling of three open strings, see (141). Thus a R-R p-brane has the coupling dependence expected for a soliton in a theory of open strings. The type II string theories, as defined so far, are theories of oriented closed strings. Consider now an extension where one adds to the theory open strings with Dirichlet boundary conditions along $\mathrm{p}$ directions. If we manage to identify the corresponding D-p-branes with the R-R p-brane solutions, this provides a description of type II string theory in these solitonic backgrounds.

\section{3 p-branes and D-branes}

Surprising as it may be, the identification of R-R p-branes and D-branes can be supported by convincing arguments. Let us compare the known properties of these objects. R-R p-branes preserve half of supersymmetry and can be located at arbitrary positions in transverse space. The same is true for D-branes with $\mathrm{p}=$ $0,2,4,6$ in type IIA and $\mathrm{p}=1,3,5$ in type IIB string theory. The corresponding Killing spinors are constant and are given by (252). The translational symmetries trivially agree. These D-p-branes are BPS states and since the central charge associated with a BPS state with Killing spinors (252) is precisely the R-R charge, they must carry R-R charge. A crucial quantitative test is to compute the R-R charge carried by a single D-p-brane. To do so one has to compute the force due to exchange of R-R gauge fields between to D-p-branes. 
One first computes an annulus diagram with Dirichlet boundary conditions on both boundaries. This diagram can be factorized in two ways: either as a sum over intermediate open strings, or a as a sum over intermediate closed strings. In the closed string channel the diagram can be visualized as a cylinder (closed string propagator) ending on the two D-branes. In this picture it is obvious that one measures the total force between the D-branes resulting from the exchange of arbitrary closed string states. This amplitude vanishes, which tells us that the total force vanishes, as expected for a BPS state. To extract the long range part of the force one takes the two D-branes to be far apart and expands the amplitudes in the masses of the closed string states. Then the exchange of massless states dominates. In detail one finds an attractive force due to graviton and dilaton exchange which is cancelled exactly by a repulsive force due to exchange of rank $(\mathrm{p}+1)$ tensor gauge fields. The static R-R forces correspond to a generalized Coulomb potential,

$$
V_{\mathrm{R}-\mathrm{R}}=\frac{Q_{p}}{r^{D-p-3}}=\frac{Q_{p}}{r^{7-p}} .
$$

It turns out that one D-p-brane carries precisely one unit of R-R p-brane charge,

$$
Q_{p}=c_{p}=\frac{(2 \pi)^{7-p}}{(7-p) \omega_{8-p}}\left(\alpha^{\prime}\right)^{\frac{7-p}{2}} g_{S}
$$

This shows that one should identify a R-R p-brane of charge $N_{p} c_{p}$ with a system of $N_{p}$ D-p-branes. People also have computed various other quantities, including the low energy scattering, absorption and emission (encoded in the so-called greybody factors) of various strings states on R-R p-branes and D-p-branes, and the low velocity interactions between p-branes and D-branes. All these test have been successful.

Since p-branes are extended supergravity solutions with non-trivial spacetime metric, whereas D-branes are defects in flat space-time, we should of course be more precise in what we mean by identification. We have seen that both kinds of objects have the same charges, tensions and low energy dynamics. They have the same space-time and supersymmetries and saturate the same BPS bound. Thus they seem to represent the same BPS state of the theory, but in different regions of the parameter space. A description in terms of $N_{p}$ D-branes works within string perturbation theory. In presence of D-branes the effective string loop counting parameter is $N_{p} g_{S}$ instead of $g_{S}$. The reason is as follows: as we have seen in section 3 each boundary component gives rise to a factor $g_{S}$ in scattering amplitudes. In a background with D-branes every boundary component can end on each of the $N_{p}$ D-branes and therefore $g_{S}$ always occures multiplied with $N_{p}$. Since we are inerested in describing macroscopic objects with large $N_{p}$, we need to impose that $N_{p} g_{S}$ is small in order to apply perturbation theory.

Thus we are in the perturbative regime if

$$
N_{p} g_{S} \ll 1
$$


Using the Schwarzschild radius (239) we see that this equivalent to

$$
r_{S} \ll \sqrt{\alpha^{\prime}},
$$

which means that the gravitational scale is much smaller then the string scale. This explains why one does not see any backreaction of the D-branes on the space-time in string perturbation theory. D-branes have a finite tension and couple to gravity, but the deviation from flat space caused by backreaction is only seen at scales of the order $r_{S}$. The only length scale occuring in string perturbation theory is $\sqrt{\alpha^{\prime}}$ and this is the minimal scale one can resolve when probing D-branes with strings.

The R-R p-branes are solutions of the type II effective actions. These are valid at string tree level and therefore we need to be in the perturbative regime (of the closed string sector), $g_{S}<1$. Morover we have neglected $\alpha^{\prime}$-corrections, which become relevant when the curvature, mesured in string units, becomes large. The condition for having small curvature is

$$
r_{S} \gg \sqrt{\alpha^{\prime}},
$$

or, equivalently

$$
N_{p} g_{S} \gg 1
$$

which is opposite to 257,258$)$. The p-brane solution is valid in the regime of the low energy effective field theory, where stringy effects can be neglected.

Between the two regimes one can interpolate by changing the string coupling $g_{S}$, while keeping the charge $N_{p}$ fixed. In general it is not clear that one can believe in the results of such interpolations. But in our case we know that the p-brane/D-brane is the object of minimal tension for the given charge. As a BPS state it sits in a special BPS multiplet. There is no mechanism compatible with supersymmetry through which this state could decay or become a non-BPS state. Besides these arguments, various quantities have been computed in both regimes and agree with one another.

In string perturbation theory one also has D-branes with $p>6$. Therefore one might wonder whether the corresponding objects also exist as p-branes. The answer is yes, though these so-called large branes have somewhat different properties than the other branes. For example the seven- and eight-brane are not flat in the transverse dimensions. The reason is that there are no harmonic functions in transverse space that become constant at infinity (this is similar to black holes in $D<4$ ). The seven-brane carries magnetic charge under the IIB R$\mathrm{R}$ scalar $A$. Its electric partner is a (-1)-brane, the $\mathrm{D}$-instanton. The eight-brane does not have a local source. It is a domain wall solution which separates regions where the IIA mass parameter (which is similar to a cosmological constant) takes different values. The nine-brane is flat space.

\subsection{Further reading}

The type II effective actions and the corresponding p-brane solutions can be found in the book [3]. For extensive reviews of BPS-branes in supergravity and string theory, see $11,47,13,12]$. 


\section{Outlook}

In this final section we give an outlook on more recent developments.

\subsection{Eleven-dimensional M-theory}

Besides the R-R charged p-branes, type II string theories contain various other BPS solutions. Since all these carry central charges of the supersymmetry algebra, they can be constructed systematically. The other string theories also have their BPS solitons. Combining perturbative string theory with the knowledge about the BPS states one can show that the strong coupling behaviour of any of the five string theories can be described consistently by a dual theory. Moreover, one can interrelate all five superstring theories by such string dualities. These dualities have not been fully proven yet, but one has compared various accessible quantities and all these tests have been successful. The dualities give a coherent picture where all perturbative string theories are limits of one single underlying theory.

This is by now a huge subject, which deserves a separate set of lectures. Here we will only illustrate it by reviewing Witten's analysis [18] of the strong coupling behaviour of type IIA string theory. Consider the spectrum of finite mass objects in IIA string theory. It starts with the massless IIA supergravity multiplet, then comes an infinite series of excited string states with masses (198)

$$
\alpha^{\prime} M^{2} \sim N
$$

where $N=1,2, \ldots$. As further finite mass objects the theory contains states with $N_{0}$ D-0-branes, with masses (242)

$$
\alpha^{\prime} M^{2} \sim \frac{N_{0}}{g_{S}} .
$$

(One can show that there are no bound states at threshold, so the states with $N_{0}>1$ are $N_{0}$-particle states.) In the perturbative regime, $g_{S} \ll 1$, the D-0-branes are very heavy. But when extrapolating to strong coupling, $g_{S} \rightarrow \infty$, they become much lighter than any perturbative excitation. Since the D-0-branes are BPS-states, we know that the mass formula (262) is not modified at strong coupling. For very large $g_{S}$ one gets a quasi-continuum of D-0-brane states above the massless supergravity multiplet. The collective modes of a D-0-brane sit in a so-called short multiplet of the IIA supersymmetry algebra. Short multiplets are special massive multiplets which saturate the BPS bound. They have less components than generic massive multiplets. The multiplet of the $\mathrm{D}$-0-brane is a massive version of the supergravity multiplet: it has the same number of states and the same spin content. Thus the low energy, strong coupling spectrum looks like the Kaluza-Klein spectrum obtained by dimensional reduction of an elevendimensional theory. The only candidate is eleven-dimensional supergravity, the unique supersymmetric theory in eleven dimensions. When comparing the low 
energy, strong coupling spectrum of IIA string theory to the Kaluza-Klein spectrum of eleven-dimensional supergravity one finds that both agree, provided one relates the string coupling to the radius $R_{11}$ of the additional space dimension according to,

$$
g_{S}^{2}=\left(\frac{R_{11}}{L_{\mathrm{Pl}}}\right)^{3}
$$

and the string scale $\alpha^{\prime}$ to the eleven-dimensional Planck length $L_{\mathrm{Pl}}$ according to:

$$
\alpha^{\prime}=\frac{L_{\mathrm{Pl}}^{3}}{R_{11}} .
$$

The eleven-dimensional Planck length is defined through the eleven-dimensional gravitational coupling by: $\kappa_{(11)}^{2}=L_{\mathrm{Pl}}^{9}$. The relation between the eleven-dimensional metric and the IIA string frame metric is:

$$
d s_{11}^{2}=e^{2 \Phi / 3}\left(d s_{\mathrm{IIA}, \mathrm{Str}}^{2}+\left(d x^{11}-A_{\mu} d x^{\mu}\right)^{2}\right)
$$

where $\Phi$ is the IIA dilaton and the Kaluza-Klein gauge field $A_{\mu}$ becomes the $\mathrm{R}-\mathrm{R}$ one form.

This indicates that the strong coupling limit of IIA string theory is an elevendimensional theory, called M-theory. We do not have enough information to give a complete definition, but we know that M-theory has eleven-dimensional supergravity as its low energy limit. There must be additional degrees of freedom, because eleven-dimensional supergravity is not consistent as a quantum theory. Even without a complete definition of M-theory, one can find more evidence for the duality. Eleven-dimensional supergravity has BPS solitons, which properly reduce under dimensional reduction to the solitons of IIA string theory. In particular it has a supersymmetric membrane solution, called the M-2-brane, which reduces to the fundamental IIA string.

\subsection{String dualities}

Let us now consider the other string theories. What about type IIB? The theory has maximal supersymmetry, and its massless spectrum cannot be obtained by dimensional reduction from a higher dimensional supersymmetric theory. The only obvious possibility is that it is selfdual, which means that the strong and weak coupling limits take the same form. One can show that inverting the coupling, $g_{S} \rightarrow g_{S}^{-1}$, preserves the form of the action and is a symmetry of the BPS spectrum, if one simultanously interchanges the fundamental IIB string with the D-1-brane. The strong coupling limit is again a IIB string theory, with solitonic strings (D-1-branes) now playing the role of the fundamental objects. The transformation relating weak and strong coupling is called S-duality and works the same way as the Montonen-Olive duality in four-dimensional $N=4$ Super-Yang-Mills theory. It has also been verified that S-duality is respected by instanton corrections to string amplitudes. 
In a similar way, the strong coupling limit of the type I string is the heterotic string with gauge group $S O(32)$, and vice versa. We already saw that both theories have the same massless spectra, while the perturbative massive spectra and interactions were different. Both theories cannot be selfdual (for example, inverting the string coupling does not preserve the form of the effective action). But once solitonic BPS states are included, the BPS spectra are equal and reversing the coupling relates the two effective actions. The heterotic $S O(32)$ string is identified with the D-1-brane of type I.

What is left is to determine the strong coupling behaviour of the $E_{8} \times E_{8}$ heterotic string. This turns out to be again eleven-dimensional M-theory but this time compactified on an interval instead of a circle. The interval has two tendimensional boundaries, on which ten-dimensional vector multiplets with gauge group $E_{8}$ are located. This is also known as Horava-Witten theory.

Let us summarize the strong-coupling limits of the five supersymmetric string theories:

\begin{tabular}{|l|l|}
\hline String theory & Strong coupling dual \\
\hline \hline IIA & M-theory on circle \\
\hline IIB & IIB \\
\hline I & Heterotic $S O(32)$ \\
\hline Heterotic $S O(32)$ & I \\
\hline Heterotic $E_{8} \times E_{8}$ & M-theory on intervall \\
\hline
\end{tabular}

These dualities fall into two classes: either one has a relation between strong and weak coupling. This is called S-duality. Or the coupling is mapped to a geometric datum, the radius of an additional dimension. There is a third type of string duality, which leads to further relations between string theories. It is called Tduality and relates weak coupling to weak coupling, while acting non-trivially on the geometry. Since weak coupling is preserved, one can check that T-duality is preserved in perturbation theory. By T-duality, the IIA string theory compactified on a circle of radius $R$ is equivalent to IIB string theory compactified on a circle of inverse radius in string length units, $\tilde{R}=\frac{\alpha^{\prime}}{R}$. One can take the decompactification limit and obtain ten-dimensional IIB theory as the zero radius limit of compactified IIA theory and vice versa. In the same way one can relate the two heterotic string theories. When acting on open strings, T-duality exchanges Neumann boundary conditions with Dirichlet boundary conditions. Therefore the T-dual of type I string theory is a theory containing open strings which are coupled to D-branes. Though one might consider this as a solitonic sector of type I theory, it is sometimes called type I' theory. Let us summarize 
the T-duals of the five supersymmetric string theories:

\begin{tabular}{|l|l|}
\hline String theory & T-dual theory \\
\hline \hline IIA & IIB \\
\hline IIB & IIA \\
\hline I & I' \\
\hline Heterotic $S O(32)$ & Heterotic $E_{8} \times E_{8}$ \\
\hline Heterotic $E_{8} \times E_{8}$ & Heterotic $S O(32)$ \\
\hline
\end{tabular}

Finally there is yet another relation between IIB string theory and type I. Type IIB has supersymmetric D-9-branes. These D-branes are space-filling, they correspond to adding open strings with Neumann boundary conditions in all directions. From our earlier discussion we know that the only consistent coupling between open and closed superstrings is a non-oriented theory with gauge group $S O(32)$, namely type I. This can be realized as a configuration in IIB string theory, where one adds 32 D-9-branes together with additional non-dynamical objects, so-called orientifold planes, which reverse world-sheet parity. Type I string theory is an 'orientifold' of type IIB. More generally, after introducing Dbranes and orientifolds, the type I, IIA and IIB string theories can be considered as one theory in different backgrounds, which can be transformed into another by T-duality and orientifolding. The type I' theory, which we introduced above as the T-dual of type I, can also be obtained as an orientifold of type IIA. Therefore type I' and type I theory are also called type IA and type IB.

Thus we see a bigger picture emerging once we include the BPS solitons of the five supersymmetric string theories. All theories are related to one another and to eleven-dimensional M-theory, and all strong couplings limits can be consistently described. Therefore one believes today that the different string theories are perturbative limits of one single underlying theory. Due to the role of D-branes and since there is an eleven-dimensional limit, which cannot be described by perturbative string theory, one prefers to call it M-theory.

\subsection{Further reading.}

String dualities and how they relate the five supersymmetric string theories to one another are discussed in the book [3] and in various lectures notes. The paper 48 gives a nice overview of the various dualities that we mentioned above. The lectures [46] approache the subject from the side of effective supergravity theories and string compactifications, whereas 44] is an introduction to supergravity which also covers branes and string dualities. Other lecture notes on string theory and string dualities are $49,50,51$.

T-duality, which we only mentioned briefly in these lectures is reviewed at length in [16]. The role of combined T- and S-dualities, then called U-dualities, in string and M-theory compactifications is reviewed in [17]. D-branes and their 
applications are discussed in 52,53. For a recent reviews of open strings, see [19]. The lectures [14] are devoted to the description of BPS black holes in string theory. They also cover the ten-dimensional brane solutions of type II string theories and how they are related by T- and S-duality. BPS solutions of elevendimensional supergravity (M-branes) and their relation to the brane solutions of type II string theory are explained in [12].

\subsection{Lightning review of further topics}

Let us finally mention areas of active research together with some references, which might be useful for the interested reader.

What is M-theory? So far M-theory was characterized by its relation to various perturbative string theories and through its eleven-dimensional low energy limit, supergravity. The fundamental open question is how to define M-theory without recourse to a particular background, perturbation theory or particular limits. The recent developments show that besides strings also various branes have to be taken into account as dynamical objects. The question which remains open is which of these objects are truly fundamental. When considering all pbranes as equally fundamental as strings, one immediately faces the problems of how to quantize higher-dimensional objects. Among p-branes, strings $(p=1)$ and particles particles $(p=0)$ are singled out, because their world-volume theories are free as long as the background geometry is flat. This underlies the power of string perturbation theory. The situation is completely different for higher-dimensional branes $(p>1)$, where the world-volume theory is a complicated interacting theory, even in a flat background. Therefore no analogon of string perturbation theory for these objects has been developed so far. Alternatively, one particular kind of brane might be the fundamental object, whereas all others are obtained by dimensional reduction or as solitons. There are two candidates for which concrete proposals have been made: the supermembrane and the D-0-brane.

The supermembrane. Eleven-dimensional supergravity has a solitonic twobrane solution, called the supermembrane or the M-2-brane. The three-dimensional action for the collective modes of this solution contains a Nambu-Goto term and Wess-Zumino term, which describe the coupling to gravity and to the threeform gauge field of eleven-dimensional supergravity. One can then try to treat this membrane as a fundamental object in an analogous way to the fundamental string in string theory. Moreover one can get back the IIA string by dimensional reduction. Supermembrane theory is much more complicated then string theory, because the world-volume theory does not become free in a flat background, as discussed above. Also note that there is no local Weyl invariance for p-branes with $p \neq 1$. Therefore there is no conformal world volume action and no analogon of the Polyakov formulation. 
At the WE-Haereus-Seminar, supermembrane theory was the subject of lectures by Hermann Nicolai. A pedagogical introduction to the subject, which also covers the relation to other approaches to M-theory is provided by his Trieste lectures [55].

Matrix theory. In the matrix theory formulation of M-theory, also called $\mathrm{M}$ (atrix) theory, the D-0-brane is the fundamental object. More precisely, there is a conjecture due to Banks, Fischler, Shenker and Susskind [22], which claims that eleven-dimensional M-theory in the infinite momentum frame is given exactly by the limit $N \rightarrow \infty$ of the supersymmetric $U(N)$ quantum mechanics describing a system of $N$ D-0-branes.

$\mathrm{M}$ (atrix) theory can be viewed as an alternative formulation of supermembrane theory, since the finite $-N-\mathrm{M}$ (atrix) model Hamiltonian is an approximation of the supermembrane Hamiltonian. In M(atrix) theory multi-membrane states are described by clusters of D-0-branes. Conversely D-0-branes are contained in supermembrane theory as Kaluza-Klein modes of the eleven-dimensional supergravity multiplet, which consists of the zero mass states of the supermembrane. Besides [55], lectures on M(atrix) theory are [56,57,58].

Black holes. While the fundamental definition of M-theory remains to be found, string theory and D-branes have been applied to a variety of problems in gravity, field theory and particle physics. One of the most prominent applications is the description of black holes through D-branes, which elaborates on the relation between D-branes and p-brane solutions discussed in section 5. Starting from p-branes in ten dimensions one can obtain four-dimensional black holes by dimensional reduction. Performing the same reduction with the corresponding D-brane configuration, one gets a description of the system where the microscopic degrees of freedom are known. This can be used to compute the entropy of the black hole: one counts the number $N$ of microstates, i.e., excitations of the system, which belong to the same macrostate, i.e., the same total energy, charge and angular momementum:

$$
S=\log N
$$

In practice the statistical entropy $N$ is evaluated asymptotically for very large black hole mass.

The result can be compared to the Bekenstein-Hawking entropy of the black hole, which is given in terms of the area $A$ of the event horizon,

$$
S_{\mathrm{BH}}=\frac{A}{4} .
$$

One finds that the two entropies agree, $S=S_{\mathrm{BH}}$, which confirms that the Dbrane picture correctly captures the microscopic degrees of freedom of the black hole 23. As mentioned above $S$ is evaluated asymptotically, but we would like to stress that the resulting $S$ matches exactly with the Bekenstein-Hawking 
entropy. This is in contrast to other approaches, where both entropies have the same dependence on paramaters, while the numerical prefactor of the statistical cannot be determined precisely.

One can also compute and compare sub-leading contributions to both entropies. Corrections to the statistical entropy have been computed for CalabiYau compactifications of type II string theory and eleven-dimensional M-theory 2526], (see also 27]). These match precisely with corrections to the macroscopic black hole entropy, which are due to higher curvature terms in the effective action [28,29]. These higher curvature terms modify the entropy in two ways. The first is an explicit modification of the black hole solution and, hence, of the area $A$. The second is a modification of the area law (269). As pointed out by R. Wald [24], the validity of the first law of black hole mechanics in presence of higher curvature terms requires a modified definition of black hole entropy. (The first law of black hole mechanics formulates the conservation of energy. It expresses adiabatic changes of the mass to changes in terms of parameters of the black hole solution.) Both effects, the explicit change of the solution and the modified definition of the entropy, change the entropy in a complicated way, but the combined correction is relatively simple and precisely matches the statistical entropy. This is reviewed in [15].

Besides entropy, the D-brane picture has been used to compute Hawking radiation and greybody factors (see [61, 44] for review and references). This is possible for branes which are close to the BPS limit. In the D-brane picture one can compute the emission, absorption and scattering of closed string states by a D-brane. Again one finds agreement with a semiclassical treatment of the corresponding black hole solutions. Note, however, that the method only applies to D-branes and p-branes which are close to the BPS limit. The generalization to generic black holes remains an open problem, though various proposals have been made. One idea, which applies to black holes without R-R charge is a correspondence principle between black holes and fundamental strings 30,31. The idea is that a black hole evaporates through Hawking radiation until its size reaches the string scale where it converts into a highly excited fundamental string. This is supported by the observation that the entropies of black holes and fundamental strings of equal mass match precisely when the Schwarzschild radius equals the string length. Another idea 32 is to use string dualities to map four(and five-)dimensional black holes to three-dimensional black holes (BTZ black holes 33). Three-dimensional gravity does not have local degrees of freedom, because the action is a total derivative. If the space-time has boundaries one gets boundary degrees of freedom which can be described by a two-dimensional conformal field theory. Treating the horizon as a boundary, this can be used to compute the statistical entropy of three-dimensional black holes [34]. The dualities that one needs to connect these three-dimensional to four-dimensional black holes are slightly more general then those mentioned so far. In particular they change the asymptotic geometry of space-time, so that one can map a higher-dimensional black hole to a lower-dimensional one (times an internal, compact space). One can argue that these transformations do not change the 
thermodynamic properties. Moreover one finds explicitly that the BekensteinHawking entropy of the four-dimensional Schwarzschild black hole is matched by the state counting of the dual three-dimensional black hole. A related approach is to use dualities to map Schwarzschild black holes to brane configurations [35. Finally, the microscopic entropy of Schwarzschild black holes has also been computed using Matrix theory, see [58] for review and references.

The most general and most promising approach to generic black holes is the AdS-CFT correspondence [38, 39, 40]. This correspondence and its generalizations relate $D$-dimensional gravitational backgrounds to $(D-1)$-dimensional field theories. One of the roots of this idea is the so-called holographic principle 36, 37], which claims that the physics beyond the horizon of a black hole can be described in terms of a field theory associated with its horizon. The D-brane picture of black holes can be viewed as a realization of this idea, because here the interior region of the black hole has disappeared, while interactions of the exterior region with the black hole are described as interactions between closed strings in the bulk with open strings on the brane. A more general version of the holographic principle is that gravity can always be described in terms of a lower dimensional field theory. The AdS-CFT correspondence, which we briefly describe below, can be viewed as an attempt to realize this idea.

More about black holes in string theory can be found in [14 and in other reviews of the topic including [59,60,61, 62, 44, 63, 15, 58 and section 14.8 of [3].

The AdS-CFT correspondence and its generalizations. The AdS-CFT correspondence is another consequence of the relation between D-branes and p-brane solutions. Its most simple version is obtained by considering a system of $N$ D-3-branes and taking the limit $\alpha^{\prime} \rightarrow 0$, while $N g_{S}$ and $R / \alpha^{\prime}$ are kept fixed. Here $g_{S}$ is the string coupling and $R$ the characteristic scale of separation between the branes. In the D-brane picture gravity and massive string excitations decouple and one is left with the effective theory of the massless open string modes, which is a four-dimensional $\mathcal{N}=4$ supersymmetric $U(N)$ gauge theory in the large $N$ limit. The corresponding limit in the p-brane regime is the near horizon limit, where the geometry takes the form $A d S^{5} \times S^{5}$. The low energy excitations are described by supergravity on $A d S^{5}$. This observation motivated Maldacena's conjecture [38]: five-dimensional supergravity on $A d S^{5}$ is a dual description of four-dimensional $\mathcal{N}=4$ supersymmetric $U(N)$ gauge theory, the later being a conformal field theory. $A d S^{5}$ has an asymptotic region which can be identified with (the conformal compactification of) four-dimensional Minkowski space. This is called the boundary, and the conformal field theory is located there. One finds a correspondence between fields $\phi\left(x_{(5)}\right)$ of the bulk supergravity theory and operators $\mathcal{O}\left(x_{(4)}\right)$ of the Yang-Mills theory on the boundary. (Here $x_{(5)}$ are coordinates on the five-dimensional bulk and $x_{(4)}$ are coordinates on the four-dimensional boundary.) A quantitative version of the conjecture, due to Gubser, Klebanov, Polyakov [39] and Witten [40], states that the generating functional for the correlators of operators $\mathcal{O}\left(x_{(4)}\right)$ with sources $\phi_{0}\left(x_{(4)}\right)$ is given by the partition function of the supergravity theory, evaluated in the background 
$\phi\left(x_{(5)}\right)$ with boundary values $\left.\phi\left(x_{5}\right)\right|_{\text {Boundary }}=\phi_{0}\left(x_{(4)}\right)$, according to:

$$
\left\langle e^{\int d^{4} x \phi_{0}\left(x_{(4)}\right) \mathcal{O}\left(x_{(4)}\right)}\right\rangle=Z\left(\phi\left(x_{(5)}\right)\right) .
$$

There are various generalizations of this basic form of the correspondence, which relate other gravitational backgrounds to other gauge theories. One particular extension of the AdS-CFT correspondence relates five-dimensional domain wall geometries to renormalization group flows in non-conformal gauge theories. In this setup the coordinate transverse to the domain wall corresponds to the energy scale of the gauge theory [41,42]. More recently, maximally supersymmetric gravitational wave backgrounds have moved to the center of interest 43 .

Extensive reviews of the AdS-CFT correspondence can be found in 44 and 45.

Brane worlds. D-branes provide a new option for model building in particle physics. One can localize some or all matter and gauge fields of the standard model on a three-brane, while gravity propagates in the higher-dimensional bulk. Such models have the interesting feature that the size of the extra dimensions can be quite large, even in the sub-mm range. Moreover one can have a fundamental (higher-dimensional) Planck scale of $1 \mathrm{TeV}$, which provides a new approach to the gauge hierarchy problem. A low gravitational scale of $1 \mathrm{TeV}$ leads to spectacular predictions, like the mass production of black holes at the LHC. Therefore brane worlds have been a main activity in the string and particle physics community over the last years. One should stress here that though $\mathrm{TeV}$ scale gravity is possible within string theory, it is not predicted.

There is a huge variety of brane world models, which range from phenomenological models to models with explicit realization in string or M-theory, see for example 64, 65, 66, 67, 68, 69. In one variant, the so-called Randall-Sundrum model (RS II model [68], to be precise), the extra dimensions are curved in such a way that gravity is confined on the brane in a similar way as matter fields. This opens the possibility of extra dimensions which a arbitrarily large, though invisible at low energies.

At the WE-Haereus Seminar brane worlds were the subject of the lectures given by I. Antoniadis and A. Barvinsky, while J. Gundlach reviewed tests of Newton's law at short distances. A nice review of mass scales and the possible sizes of extra dimensions in string theory can be found in [20]. Experimental signatures of large extra dimensions are discussed in [21]. One particular type of brane worlds, which occure in Calabi-Yau compactifications of Horava-Witten theory, is reviewed in [70]. The lectures [72] give an introductions brane worlds and warped compactifications.

Compactifications and phenomenology. D-branes and p-branes have considerably extended the framework of string compactifications, which aim to explaine how our four-dimensional world is embedded into the fundamental tenor eleven-dimensional theory. Whereas ten years ago string phenomenology was 
synonymous with the study of the heterotic $E_{8} \times E_{8}$ string, compactified on complex three-dimensional Calabi-Yau manifolds, one now has various other options to consider. Besides brane worlds one can study compactifications where part of the standard model particles are not string modes but descend from p-branes wrapped on internal p-cycles. Switching on background fluxes of antisymmetric tensor fields, one obtains warped compactifications, where the characteristic length scale of four-dimensional space-time becomes dependent on the position in the internal space. A particular class of non-perturbative IIB backgrounds can be described purely geometrically in terms of so-called F-Theory.

The central problem of string compactifications is still the problem of vacuum degeneracy. As we have seen, the vacuum expectation value of the dilaton is not fixed at string tree level. In supersymmetric theories this holds to all orders in perturbation theory. Similarly, string compactifications in general have several scalar fields, called moduli, which parametrize the shape and size of the internal manifold and enter into the couplings of the effective field theory. The vacuum expectation values of these fields are not fixed, as long as supersymmetry is unbroken. This ruins the predictive power that the theory has in principle, and leads to continuous families of degenerate vacua. Once supersymmetry is broken the moduli get fixed, but there is a number of issues to be addressed: one needs to understand the dynamical mechanism behind supersymmetry breaking, which requires to understand the theory non-perturbatively. The potential generated for the dilaton and for the moduli should have stable vacua and no runaway behaviour. One needs sufficently large masses or sufficiently small couplings for the moduli to avoid contradiction with empirical data. Moreover, in string theory supersymmetry is closely related to the absence of the tachyon, which one does not want to reintroduce. One also wants that supersymmetry breaking occurs at a specific scale, the most popular scenario being low energy supersymmetry where the supersymmetric partners have masses of about $1 \mathrm{TeV}$. D-branes, pbranes and other new developments have added a variety of new ways to address these problems, but a definite solution remains to be found.

String compactifications on Calabi-Yau manifolds are reviewed in 71]. Lectures on warped compactifications and brane worlds can be found in [72]. Ftheory is for example explained in 49 . For an introduction to string and Mtheory particle phenomenology, see for example 73,74 .

Geometric and D-brane engineering, D-branes and non-commutative field theory. In addition to the AdS-CFT correspondence, string theory has lead to other new approaches to gauge theories and other field theories. In geometric engineering [77] one starts from branes wrapped on cycles in an internal space, which typically is a Calabi-Yau manifold, whereas in D-brane engineering 78 one studies D-brane configurations in a non-compact space-time. In both cases one takes a low energy limit (similar to the one discussed above in the context of the AdS-CFT correspondence) to decouple gravity.

Another direction stimulated by string theory and D-branes is gauge theory on non-commutative space-times. As mentioned in the lectures, the effective 
action for a D-brane is of Born-Infeld type. It has been argued that this can be reformulated as a Yang-Mills theory on a non-commutative world volume, with a deformation parameter which is determined by the bulk $B_{\mu \nu}$ field of the closed string sector [79].

Geometric engineering is reviewed in 75,76 , while gauge theory on noncommutative space-times is reviewed in [30]. For extensive lectures on D-branes, see [52,53].

Cosmology. Whereas string compactifications ususally aim at finding fourdimensional Minkowski space with a realistic particle spectrum from string theory, one should of course try to do better. Cosmological solutions of string theory should shed light on the issue of the initial singularity, describe an inflationary phase (or an alternative mechanism which takes care of the problems of the old hot big bang model), further describe the post-inflationary phase and explain the smallness of the cosmological constant. These problems have been mostly neglected by string theorists for a long time, but nowadays they find increasing interest, due to both new cosmological data and new theoretical developments. In particular branes have been invoked for either providing the mechanism for inflation or for providing an alternative to inflation.

Reviews of string cosmology can be found in $81,82,83$.

The challenge from de Sitter space. Since there is empirical evidence in favour of a small, positive cosmological constant, there has been a considerable interest in string theory in de Sitter space over the last few years. De Sitter space is a challenge for several reasons. First, most successful applications of string theory to gravity depend on supersymmetry, but supersymmetry is completely broken in presence of a positive cosmological constant. Second, de Sitter space has cosmological horizons, and the perturbative formalism which works for Minkowski space as explained in section 3 cannot be applied. Therefore de Sitter space requires a significant step beyond that framework. For a review see [54.

Tachyon condensation and string field theory. As observed several times in these lectures, the appearence of tachyons is a generic feature of string theories when there is no supersymmetry. Since the mass squared of a scalar particle is given by the curvature of its potential at the stationary point one is expanding around, this shows that one tries to expand the theory around a local maximum of the potential. Depending on the global form of the potential, the theory might be unstable, or it might be that the scalar field rolls to a minimum. This is referred to as tachyon condensation.

Tachyons do not only occure in the groundstate of bosonic string theories, but also in D-branes configurations which are not BPS states (non-BPS D-branes systems are reviewed in 84 ). Work starting with a paper by A. Sen 85 provided strong evidence that tachyon condensation occurs in unstable non-BPS configurations of D-branes. Such systems have tachyonic open string states which 
condense. The resulting stable vacuum is the closed string vacuum, whereas the D-branes have decayed and therefore open strings are absent. This work makes use of string field theory, which for a long time was mostly neglected, because it is very complicated and was believed of little practical use. The renewed interest in string field theory might bring us one step forward towards a non-perturbative and background-independent formulation of M-theory.

\section{Acknowledgements}

First of all I would like to thank the organisers of the 271-th WE-Haereus seminar for organizing this very lively and stimulating school. I would also like to thank the participants for their numerous questions and remarks, which I have tried to take into account when writing these lecture notes. The same applies to all the participants of my string theory course at the university of Jena. My special

thanks goes to Frank Saueressig and Christoph Mayer for their very helpful comments on the manuscript.

\section{References}

1. M.B. Green, J.H. Schwarz and E. Witten: Superstring Theory (Cambridge University Press, Cambrigde 1984)

2. D. Lüst and S. Theisen: Lectures on String Theory (Springer, Berlin Heidelberg 1989)

3. J. Polchinski: String Theory (Cambridge University Press, Cambridge 1998)

4. M. Kaku: Introduction to Superstrings and M-Theory (Springer, Berlin Heidelberg 2000)

5. M. Kaku: Conformal Fields and M-Theory (Springer, Berlin Heidelberg 2000)

6. P. Ginsparg: 'Applied Conformal Field Theory'. In: Fields, Strings and Critical Phenomena, Les Houches Summer School in Theoretical Physics, Session 49, Les Houches, France, June 28-August 5, 1988, ed. by E. Brezin and J. Zinn-Justin (North-Holland, Amsterdam 1990)

7. E. D'Hoker and D.H. Phong: Rev. Mod. Phys. 60, 917 (1988)

8. S. Weinberg: 'Covariant Path-Integral Approach to String Theory'. In: Strings and Superstrings, 3rd Jerusalem Winter School for Theoretical Physics, Jerusalem, Israel, December 30, 1985-January 9, 1986, ed. by S. Weinberg and T. Piran (World Scientific, Singapore 1988)

9. S. Albeverio, J. Jost, S. Paycha and S. Scarlatti: A Mathematical Introduction to String Theory (Cambridge University Press, Cambridge 1997)

10. M. Schlichenmaier: An Introduction to Riemann Surfaces, Algebraic Curves and Moduli Spaces (Springer, Berlin Heidelberg 1989)

11. K.S. Stelle: 'BPS Branes in Supergravity'. In: High Energy Physics and Cosmology 1997, ICTP Trieste Workshop, Trieste, Italy, June 2-July 11, 1997. ed. by E. Gava, A. Masiero, K.S. Narain, S. Randjbar-Daemi, G. Senjanovic, A. Smirnov, Q. Shafi. (World Scientific, Singapore 1998). Preprint: hep-th/9803116

12. P.K. Townsend: 'M-Theory from its Superalgebra'. In: Strings, Branes and Dualities, NATO Advanced Study Institute, Cargese, France, May 26-June 14, 1997, ed. by L. Beaulieu, P. DiFrancesco, M. Douglas, V. Kazakov, M. Picco, P. Windey. (Kluwer, Dordrecht 1999). Preprint: hep-th/9712004 
13. M.J. Duff: 'Supermembranes'. Preprint: hep-th/9611203

14. T. Mohaupt: Class. Quant. Grav. 17, 3429 (2000)

15. T. Mohaupt: Fortschr. Phys. 49, 1 (2001)

16. A. Giveon, M. Porrati and E. Rabinovici, Phys. Rept. 24444 (1994). Preprint: hep-th/9401139

17. N.A. Obers and B. Pioline, Phys. Rept. 318, 113 (1999). Preprint: hep-th/9809039

18. E. Witten, Nucl. Phys. B433 85 (1995). Preprint: hep-th/9503124

19. C. Angelantonj and A Sagnotti: 'Open Strings', Preprint: hep-th/0204089

20. I. Antoniadis: 'Mass Scales in String and M-Theory'. In: Superstrings and Related Matters, ICTP Trieste Workshop, Trieste, Italy, March 22-30, 1999, ed. by B. Green, J. Louis, K.S. Narain, S. Randjbar-Daemi (World Scientific, Singapore 2000)

21. I. Antoniadis and K. Benakli, Int. J. Mod. Phys. A15, 4237 (2000). Preprint: hep-ph/0007226

22. T. Banks, W. Fischler, S.H. Shenker and L. Susskind, Phys. Rev. D55, 5112 (1997) hep-th/9610043

23. A. Strominger and C. Vafa, Phys. Lett. B379, 99 (1996)

24. R.M. Wald, Phys. Rev. D48, 3427 (1993)

25. J. Maldacena, A. Strominger and E. Witten, JHEP 9712, 002 (1997)

26. C. Vafa, Adv. Theor. Math. Phys. 2, 207 (1998)

27. G.L. Cardoso, B. de Wit and T. Mohaupt, Nucl. Phys. B567, 87 (2000)

28. G.L. Cardoso, B. de Wit and T. Mohaupt, Phys. Lett. B451, 309 (1999)

29. G.L. Cardoso, B. de Wit, J. Käppeli and T. Mohaupt, JHEP 0012, 019 (2000)

30. L. Susskind: 'Some Speculations about Black Hole Entropy in String Theory'. Preprint: hep-th/9309145

31. G.T. Horowitz and J. Polchinski, Phys. Rev. D55, 6189 (1997)

32. K. Sfetsos and K. Skenderis, Nucl. Phys. B517, 179 (1998)

33. M. Banados, C. Teitelboim and J. Zanelli, Phys. Rev. Lett. 69, 1849 (1992)

34. S. Carlip, Phys. Rev. D51, 632 (1995), Phys. Rev. D55, 878 (1997)

35. R. Argurio, F. Englert and L. Houart, Phys. Lett. B426, 275 (1998)

36. G. 't Hooft: 'Dimensional Reduction in Quantum Gravity'. Preprint: gr-qc/9310026

37. L. Susskind, J. Math. Phys. 36, 6377 (1995)

38. J. Maldacena, Adv. Theor. Math. Phys. 2, 231 (1998). Preprint: hep-th/9711200

39. S.S. Gubser, I.R. Klebanov and A.M. Polyakov, Phys. Lett. B248, 105 (1998). Preprint: hep-th/9802109

40. E. Witten, Adv. Math. Phys. 2, 253 (1998). Preprint: hep-th/9802150

41. H. Itzhaki, J.M. Maldacena, J. Sonnenschein and S. Yankielowicz, Phys. Lett. B432, 298 (1998). Preprint: hep-th/9802042

42. H.J. Boonstra, K. Skenderis and P.K. Townsend, JHEP 9901, 003 (1999). Preprint: hep-th/9807137

43. D. Berenstein, J.M. Maldacena and H. Nastase, JHEP 0204, 013 (2002). Preprint: hep-th/0202021

44. O. Aharony, S.G. Gubser, J. Maldacena, H. Ooguri and Y. Oz: Phys. Rept. 323, $183(2000)$

45. E. D'Hoker and D.Z. Freedman: 'Supersymmetric Gauge Theories and the AdS/CFT Correspondence'. Preprint: hep-th/0201252

46. B. de Wit and J. Louis: 'Supersymmetry and Dualities in Various Dimensions'. In: Strings, Branes and Dualities, NATO Advanced Study Institute, Cargese, France, May 26-June 14, 1997, ed. by L. Baulieu, P. Di Francesco, M. Douglas, V. Kazakov, M. Picco, P. Windey. (Kluwer, Dordrecht 1999) 
47. P.C. West: 'Supergravity, Brane Dynamics and String Duality'. In: Nonperturbative Aspects of Strings, Branes and Supersymmetry, ICTP Conference on Super Five Brane Physics in 5+1 Dimensions, Trieste, Italy, April 1-3, 1998, ed. by M. Duff, E. Sezgin, C.N. Pope, B. Greene, J. Louis, K.S. Narain, S. Randjbar-Daemi, G. Thompson (World Scientific, Singapore 1999)

48. M. Haack, B. Körs and D. Lüst: 'Recent Developments in String Theory: From Perturbative Dualities to M-Theory'. Lectures given at the 4th National Summer School for Graduate Students of Theoretical Physics, Saalburg, Germany, August 31-September 11, 1998. Preprint: hep-th/9904033

49. A. Sen: 'An Introduction to Non-Perturbative String Theory'. In:Duality and Supersymmetric Theories, Newton Institute Euroconference, Cambridge, England, April 7-18, 1997, ed. by D.I. Olive, P.C. West (Cambridge University Press, Cambridge 1999). Preprint: hep-th/9802051

50. E. Kiritsis: 'Supersymmetry and Duality in Field Theory and String Theory'. In: Particle Physics: Ideas and Recent Developments, NATO Advanced Study Institute, Cargese, France, July 26-August 7, 1999, ed. by J.-J. Aubert, R. Gastmans, J.-M. Gaerard, (Kluwer, Dordrecht 2000). Preprint: hep-ph/9911525.

E. Kiritsis: 'Introduction to Superstring Theory'. In: Leuven Notes in Mathematical and Theoretical Physics. B9, (University of Leuven Press, Leuven 1998). Preprint: hep-th/9709062.

E. Kiritsis: 'Introduction to Non-Perturbative String Theory'. In: Trends on Theoretical Physics, CERN-Santiago De Compostela-La Plata Meeting on Trends in Theoretical Physics, La Plata, Argentina, April 28-May 6, 1997, ed. by H. Falomir, R.E. Gomboa Saravi, F.A. Schaposnik, (Amer. Inst. Phys. Woodbury, 1998). Preprint: hep-th/9708130

51. R.J. Szabo: 'Busstepp Lectures on String Theory'. Preprint: hep-th/0207142

52. C.P. Bachas: 'Lectures on D-Branes'. In: Proceedings of the Newton Euroconference on Duality and Supersymmetric Theories, April 7-18, 1997, Cambridge, England, ed. by D.I. Olive, P.C. West (Cambridge University Press, Cambridge 1999)

53. C.V. Johnson: 'D-Brane Primer'. Preprint: hep-th/0007170

54. M. Spradlin, A. Strominger and A. Volovich, 'Les Houches Lectures on De Sitter Space'. Preprint: hep-th/0110087

55. H. Nicolai and R. Helling: 'Supermembranes and M(atrix) Theory'. In: ICTP Spring School on Nonperturbative Aspects of String Theory and Supersymmetric Gauge Theories, Trieste, Italy, March 23-31, 1998, ed. by M. Duff, E. Sezgin, C.N. Pope, B. Greene, J. Louis, K.S. Narain, S. Randjbar-Daemi, G. Thompson (World Scientific, Singapore 1999). Preprint: hep-th/9809103

56. A. Bilal: 'M(atrix) Theory: a Paedagogical Introduction'. In: Meeting on Quantum Aspects of Gauge Theories, Supersymmetry and Unification, Neuchatel, Switzerland, September 18-23, 199\%, ed. by J.-P. Derendinger, C. Lucchesi, (Wiley-VHC, Weinheim 1997) (Fortschr. Phys. 471 (1999)). Preprint: hep-th/9710136

57. T. Banks, Nucl. Phys. Proc. Suppl. 67180 (1998). Preprint: hep-th/9710231

58. D. Bigatti and L. Susskind: 'Review of Matrix Theory'. In: Strings, Branes and Dualities, NATO Advanced Study Institute, Cargese, France, May 26-June 14, 1997, ed. by L. Baulieu, P. Di Francesco, M. Douglas, V. Kazakov, M. Picco, P. Windey. (Kluwer, Dordrecht 1999). Preprint: hep-th/9712072

59. R. D'Auria and P. Fré: 'BPS Black Holes in Supergravity'. Preprint: hep$\operatorname{th} / 9812160$

60. D. Youm, Phys. Rept. 316, 1 (1999). Preprint: hep-th/9710046

61. J. Maldacena: Black Holes in String Theory. PhD Thesis, Princeton University, Princeton (1996). Preprint: hep-th/9607235 
62. G. Mandal: 'A Review of the D1/D5 System and Five Dimensional Black Hole from Supergravity and Brane Viewpoints'. Preprint: hep-th/0002184

63. K. Skenderis: 'Black Holes and Branes in String Theory'. Preprint: hep-th/9901050

64. V. Rubakov and M. Shaposhnikov, Phys. Lett. B125, 136 (1983)

65. N. Arkani-Hamed, S. Dimopoulos and G. Dvali, Phys. Rev. D59, 086004 (1999)

66. I. Antoniadis, N. Arkani-Hamed, S. Dimopoulos and G. Dvali, Phys. Lett. B436, 257 (1998)

67. L. Randall and R. Sundrum, Phys. Rev. Lett. 83, 3370 (1999)

68. L. Randall and R. Sundrum, Phys. Rev. Lett. 83, 4690 (1999)

69. A. Lukas, B.A. Ovrut, K.S. Stelle and S. Waldram, Phys. Rev. D59, 086001 (1999), Nucl. Phys. B552, 246 (1999)

70. B. Ovrut: ' $N=1$ Supersymmetric Vacua in Heterotic M-Theory'. Preprint: hepth/9915115

71. B.R. Greene: 'String Theory on Calabi-Yau Manifolds'. In: Fields, Strings and Duality, TASI 1996, Boulder, USA, June 2-28, 1996, ed. by C. Efthimiou, B. Greene (World Scientific, Singapore 1997). Preprint: hep-th/9702155

72. S. Kachru: 'Lectures on Warped Compactifications and Stringy Brane Constructions'. In: Strings, Branes, and Gravity, TASI 1999, Boulder, USA, May 31-June 25, 1999, ed. by J. Harvey, S. Kachru, E. Silverstein, R. Edge, (World Scientific, Singapore 2001). Preprint: hep-th/0009247

73. J. Louis: 'Phenomenological Aspects of String Theory'. In: Nonperturbative Aspects of String Theory and Supersymmetric Gauge Theories, ICTP Spring School, Trieste, Italy, March 23-31, 1998, ed. by M. Duff, E. Sezgin, C.N. Pope, B. Greene, J. Louis, K.S. Narain, S. Randjbar-Daeme, G. Thompson (World Scientific, Singapore 1999)

74. M. Dine: 'TASI Lectures on M Theory Phenomenology. In: String, Branes, and Gravity, TASI 99, Boulder, USA, May 31-June 25, 1999, ed. by J. Harvey, S. Kachru, E. Silverstein, R. Egde (World Scientific, Singapore 2001). Preprint: hepth/0003175

75. A. Klemm, 'On the Geometry behind $N=2$ Supersymmetric Effective Actions in Four Dimensions'. In: Duality - Strings and Fields, 33rd Karpacz Winter School of Theoretical Physics, Karpacz, Poland, February 13-22, 1997, ed. by Z. Hasiewicz, Z. Jaskolski, J. Sobczyk (North-Holland, Amsterdam 1998). Preprint: hep-th/9705131

76. P. Mayr, 'Geometric Construction of $N=2$ Gauge Theories'. In: Meeting on Quantum Aspects of Gauge Theories, Supersymmetry and Unification, Neuchatel, Switzerland, September 18-23, 1997, ed. by J.-P. Derendinger, C. Lucchesi, (WileyVHC, Weinheim 1997) (Fortschr. Phys. 471 (1999)). Preprint: hep-th/9807096

77. A. Klemm, W. Lerche, P. Mayr, C. Vafa and N. Warner, Nucl. Phys. B477, 746 (1996). Preprint: hep-th/9604034.

S. Katz, A. Klemm and C. Vafa, Nucl. Phys. B497, 746 (1997). Preprint: hepth/9609239.

S. Katz, P. Mayr and C. Vafa, Adv. Theor. Math. Phys. 1, 53 (1998). Preprint: hep-th/9706110

78. A. Hanany and E. Witten, Nucl. Phys. B492, 152 (1997). Preprint: hep-th/9611230

79. N. Seiberg and E. Witten, JHEP 09, 032 (1999)

80. M.R. Douglas and N.A. Nekrasov, Rev. Mod. Phys. 73977 (2002). Preprint: hepth/0106048

81. J.E. Lidsey, D. Wands and E.J. Copeland, Phys. Rept. 337343 (2000). Preprint: hep-th/9909061

82. D.A. Easson, Int. Jour. Mod. Phys. A16 4803 (2001). Preprint: hep-th/0003086 
83. M. Gasperini and G. Veneziano: 'The Pre-Big Bang Scenario in String Cosmology'. Preprint: hep-th/0207130

84. J.H. Schwarz: 'TASI Lectures on Non-BPS D-Branes Systemes'. In: Strings, Branes, and Gravity, TASI 99, Boulder, USA, May 31-June 25, 1999, ed. by J. Harvey, S. Kachru, E. Silverstein, R. Edge (Worlscientific, Singapore 2001). Preprint: hep-th/9908144

85. A. Sen: 'Tachyon Condensation on the Brane Antibrane System', JHEP 9809, 023 (1998). Preprint: hep-th/9805170 